Supporting Information for

\title{
Molybdenum Disubstituted Alkylidene Complexes
}

\author{
Jordan W. Taylor, Richard R. Schrock, * and Charlene Tsay \\ Department of Chemistry, University of California-Riverside, Riverside, CA 92501 \\ Email: richard.schrock@ucr.edu
}

\section{Contents}

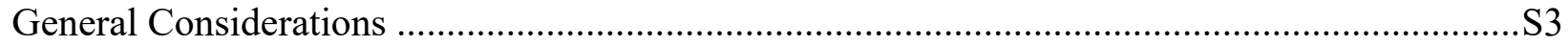

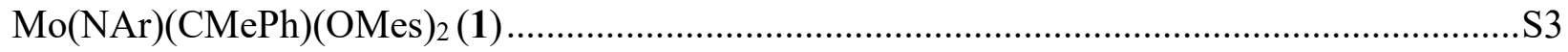

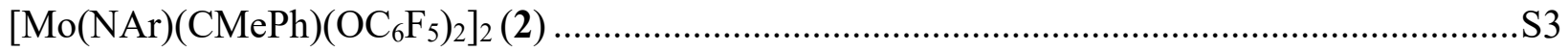

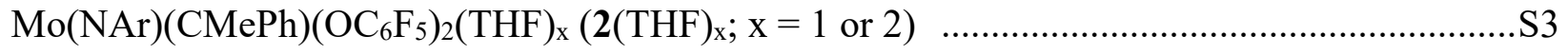

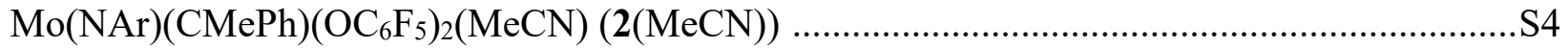

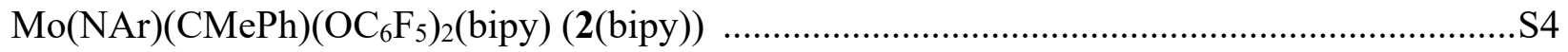

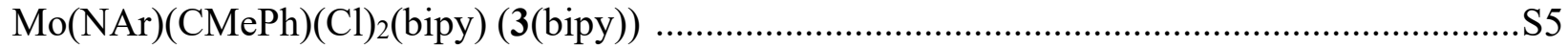

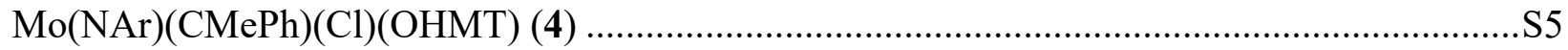

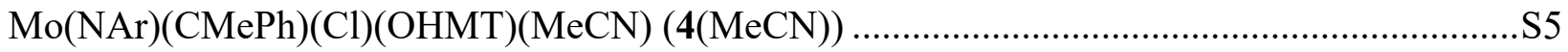

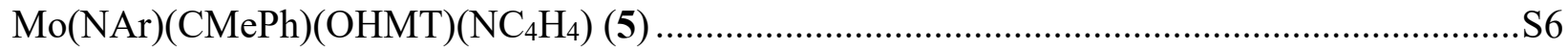

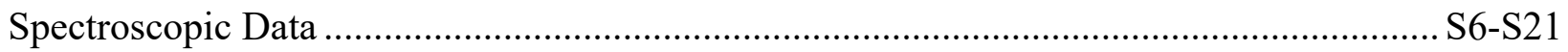

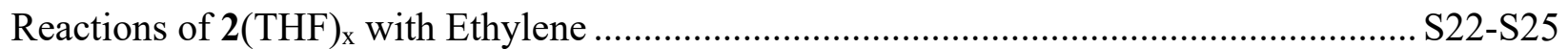

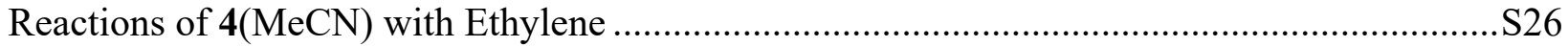

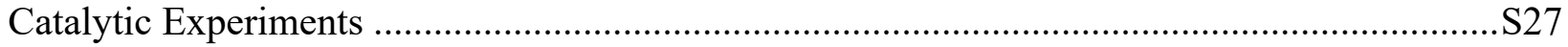

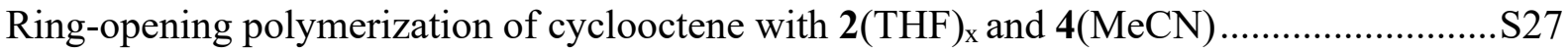

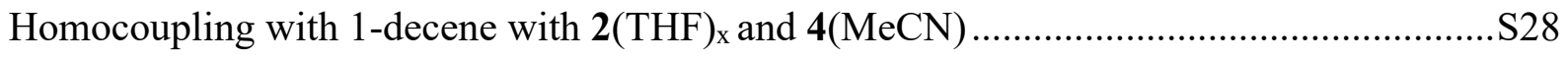

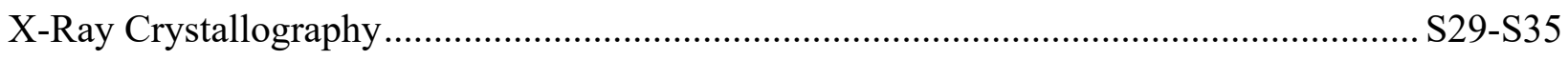

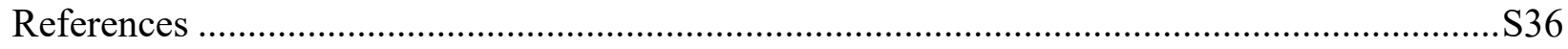


General considerations. Unless otherwise noted, all manipulations were carried out using standard Schlenk or glovebox techniques under a $\mathrm{N}_{2}$ atmosphere. THF, $\mathrm{Et}_{2} \mathrm{O}$, hexanes, benzene, toluene, and acetonitrile were dried and deoxygenated by passage through activated alumina in a solvent purification system from JC Meyer Solvent Systems followed by storage over $4 \AA$ molecular sieves. Non-halogenated and non-nitrile containing solvents were tested with a standard solution of purple sodium benzophenone ketyl in THF to confirm effective oxygen and moisture removal prior to use. Pentafluorophenol $\left(\mathrm{C}_{6} \mathrm{~F}_{5} \mathrm{OH}\right), 2$,4,6-trimethylphenol $(\mathrm{MesOH}), \alpha-$ methylstyrene, 2,2'-bipyridine (bipy) and trimethylchlorosilane (TMSCl) were purchased from commercial suppliers and purified according to literature procedures prior to use. Sodium pyrrolide $\left(\mathrm{NaNC}_{4} \mathrm{H}_{4}\right),{ }^{1} \quad$ lithium-hexamethylterphenoxide $(\mathrm{LiOHMT}=$ LiO-2,6-(2,4,6$\left.\left.\left.\mathrm{Me}_{3} \mathrm{C}_{6} \mathrm{H}_{2}\right)_{2} \mathrm{C}_{6} \mathrm{H}_{3}\right)\right),{ }^{2} \mathrm{Mo}(\mathrm{NAr})\left(\mathrm{CHCMe}_{3}\right)\left(\mathrm{OR}_{\mathrm{F} 6}\right)_{2}{ }^{3}$ and $\mathrm{Mo}(\mathrm{NAr})\left(\mathrm{CHMe}_{2} \mathrm{Ph}\right)\left(\mathrm{OR}_{\mathrm{F} 6}\right)_{2}{ }^{3}\left(\mathrm{OR}_{\mathrm{F} 6}=\right.$ $\left.\mathrm{OC}\left(\mathrm{CH}_{3}\right)\left(\mathrm{CF}_{3}\right)_{2}\right)$ were prepared according to literature procedures. Elemental analyses were performed by Midwest Microlab, LLC, Indianapolis, IN. Deuterated solvents were purchased from Cambridge Isotope Laboratories Inc., degassed, and dried over activated $4 \AA$ molecular sieves for at least $24 \mathrm{~h}$ prior to use. NMR spectra were recorded on Bruker Avance $600 \mathrm{MHz}$ and Bruker Avance $300 \mathrm{MHz}$ spectrometers. ${ }^{1} \mathrm{H}$ and ${ }^{13} \mathrm{C}$ chemical shifts are reported in ppm relative to tetramethylsilane using residual solvent as an internal standard. ${ }^{19} \mathrm{~F}$ chemical shifts are reported in ppm relative to fluorobenzene as an external standard.

Mo(NAr)(CMePh)(OMes)2 (1). $\alpha$-Methylstyrene (0.014 g, $0.12 \mathrm{mmol})$ was added to a solution of $\mathrm{Mo}(\mathrm{NAr})\left(\mathrm{CHCMe}_{3}\right)\left(\mathrm{OR}_{\mathrm{F} 6}\right)_{2}(0.042 \mathrm{~g}, 0.060 \mathrm{mmol})$ in benzene $(5 \mathrm{~mL})$ and the vessel was sealed in a $50 \mathrm{~mL}$ Schlenk flask with Teflon stopper. The reaction was subjected to three freeze-pump-thaw cycles prior to being heated to $60{ }^{\circ} \mathrm{C}$ for 5 hours. Every 1 hour, the Teflon stopper was opened to vacuum $\left(10^{-2}\right.$ mbar $)$ briefly prior to continuing heating. After $5 \mathrm{~h}$, the volatiles were removed in vacuo. The dark red residue was extracted with pentane $(3 \times 1 \mathrm{~mL})$ and extract was filtered through Celite. Volatiles were again removed in vacuo prior to dissolving the residue in benzene $(2 \mathrm{~mL})$ and adding 2,4,6-trimethylphenol $(0.016 \mathrm{~g}, 0.12 \mathrm{mmol})$ as a benzene $(0.5 \mathrm{~mL})$ solution. The reaction was stirred $1 \mathrm{~h}$ prior to removing volatiles in vacuo. The red residue was dissolved in minimal pentane $(\sim 0.3 \mathrm{~mL})$ and the solution was left at $-30{ }^{\circ} \mathrm{C}$ overnight during which time red plates grew. The crystals were rinsed with chilled pentane $\left(-30{ }^{\circ} \mathrm{C}, 0.3 \mathrm{~mL}\right)$ and dried in vacuo: yield $0.029 \mathrm{~g}(74 \%)$. A single crystal suitable for XRD was obtained by cooling a saturated pentane solution to $-30{ }^{\circ} \mathrm{C}:{ }^{1} \mathrm{H}$ NMR $\left(600 \mathrm{MHz}, \mathrm{C}_{6} \mathrm{D}_{6}\right) \delta$ 7.3-7.4 (m, 3H), $7.0-6.9(\mathrm{~m}$, $5 \mathrm{H}), 6.8(\mathrm{~s}, 4 \mathrm{H}), 3.5(\mathrm{dq}, J=13.7,6.8 \mathrm{~Hz}, 2 \mathrm{H}), 3.0(\mathrm{~s}, 3 \mathrm{H}), 2.3(\mathrm{~s}, 12 \mathrm{H}), 2.2(\mathrm{~s}, 6 \mathrm{H}), 1.0(\mathrm{~d}, J=$ $6.8 \mathrm{~Hz}, 12 \mathrm{H}) ;{ }^{13} \mathrm{C}\left\{{ }^{1} \mathrm{H}\right\} \mathrm{NMR}\left(151 \mathrm{MHz}, \mathrm{C}_{6} \mathrm{D}_{6}\right) \delta 263.1,159.7,153.6,147.3,146.0,130.3,129.3$, 128.6, 128.4, 127.6, 126.4, 126.2, 123.4, 29.1, 23.5, 20.8, 17.4, 16.9. Anal. Calcd. for $\mathrm{C}_{38} \mathrm{H}_{47} \mathrm{MoNO}_{2}\left(+1 \mathrm{C}_{7} \mathrm{H}_{8}\right): \mathrm{C}, 73.25 ; \mathrm{H}, 7.51 ; \mathrm{N}, 1.90$. Found: C, 73.58; H, 7.77; N, 1.98 .

[Mo(NAr) $\left.(\mathbf{C M e P h})\left(\mathbf{O C}_{6} \mathbf{F}_{5}\right)_{2}\right]_{2}$ (2). $\alpha$-Methylstyrene $(0.466 \mathrm{~g}, 3.95 \mathrm{mmol})$ was added to a solution of $\mathrm{Mo}(\mathrm{NAr})\left(\mathrm{CHMe}_{2} \mathrm{Ph}\right)\left(\mathrm{OR}_{\mathrm{F} 6}\right)_{2}(0.604 \mathrm{~g}, 0.789 \mathrm{mmol})$ in $\mathrm{Et}_{2} \mathrm{O}(7 \mathrm{~mL})$. The solution was stirred for 5 minutes and a solution of pentafluorophenol $(0.312 \mathrm{~g}, 1.66 \mathrm{mmol})$ in $\mathrm{Et}_{2} \mathrm{O}(2 \mathrm{~mL})$ was added dropwise. After 4 hours, the yellow precipitate was filtered off, washed with $\mathrm{Et}_{2} \mathrm{O}(4 \times 1$ $\mathrm{mL})$, and dried in vacuo: yield $0.572 \mathrm{~g}(95 \%)$. [Mo(NAr) $\left.(\mathrm{CMePh})\left(\mathrm{OC}_{6} \mathrm{~F}_{5}\right)_{2}\right]_{2}$ is not soluble enough in $\mathrm{CD}_{2} \mathrm{Cl}_{2}$ or $\mathrm{CDCl}_{3}$ to acquire NMR data readily. Anal. Calcd. for $\mathrm{C}_{32} \mathrm{H}_{25} \mathrm{~F}_{10} \mathrm{MoNO}_{2}$ : $\mathrm{C}$, 51.84; H, 3.40; N, 1.89. Found: C, 51.81; H, 3.75; N, 1.62 .

Mo(NAr)(CMePh)(OC $\left.6 \mathbf{F}_{5}\right)_{2}$ (THF) $)_{\mathbf{x}}\left(\mathbf{2}(\mathrm{THF})_{\mathrm{x}} ; \mathrm{x}=1\right.$ or 2). $\left[\mathrm{Mo}(\mathrm{NAr})(\mathrm{CMePh})\left(\mathrm{OC}_{6} \mathrm{~F}_{5}\right)_{2}\right]_{2}$ $(0.007 \mathrm{~g}, 0.0094 \mathrm{mmol})$ was dissolved in THF- $d_{8}(0.6 \mathrm{~mL})$ to give an orange solution: ${ }^{1} \mathrm{H} \mathrm{NMR}$ 
$\left(600 \mathrm{MHz}, \mathrm{THF}-d_{8},-40{ }^{\circ} \mathrm{C}\right) \delta 7.4(\mathrm{~d}, J=7.7 \mathrm{~Hz}, 2 \mathrm{H}), 7.3(\mathrm{t}, J=7.7 \mathrm{~Hz}, 2 \mathrm{H}), 7.2(\mathrm{~s}, 3 \mathrm{H}), 7.1(\mathrm{t}, J$ $=7.3 \mathrm{~Hz}, 1 \mathrm{H}), 3.8$ (hept, $J=6.1,5.6 \mathrm{~Hz}, 2 \mathrm{H}), 3.7(\mathrm{~s}, 2 \mathrm{H}), 3.6(\mathrm{~s}, 3 \mathrm{H}), 1.7(\mathrm{~s}, 2 \mathrm{H}), 1.1(\mathrm{dd}, J=10.1$, $7.0 \mathrm{~Hz}, 12 \mathrm{H}) ;{ }^{19} \mathrm{~F}\left\{{ }^{1} \mathrm{H}\right\} \mathrm{NMR}\left(564 \mathrm{MHz}, \mathrm{THF}-d_{8},-40{ }^{\circ} \mathrm{C}\right)$ Major Isomer: $\delta-157.5(\mathrm{~d}, J=21.7 \mathrm{~Hz})$, $-158.3(\mathrm{~d}, J=21.9 \mathrm{~Hz}),-164.8(\mathrm{t}, J=21.7 \mathrm{~Hz}),-165.1(\mathrm{t}, J=21.7 \mathrm{~Hz}),-172.2(\mathrm{~d}, J=22.9 \mathrm{~Hz})$, $-172.3(\mathrm{dd}, J=45.6,23.5 \mathrm{~Hz}) ;{ }^{19} \mathrm{~F}\left\{{ }^{1} \mathrm{H}\right\} \mathrm{NMR}\left(564 \mathrm{MHz}, \mathrm{THF}-d_{8}, 22{ }^{\circ} \mathrm{C}\right) \delta-156.2,-157.1,-$ $163.7,-163.9,-170.8,-171.1 ;{ }^{13} \mathrm{C}\left\{{ }^{1} \mathrm{H}\right\} \mathrm{NMR}\left(151 \mathrm{MHz}, \mathrm{THF}-d_{8},-40{ }^{\circ} \mathrm{C}\right) \delta 310.7,152.3,148.2$, $144.5,142.6,142.0,141.7(\mathrm{t}, J=13.0 \mathrm{~Hz}), 141.0,140.5,139.8,139.4,138.1,137.8,134.6(\mathrm{~d}, J=$ 14.6 Hz), 133.0, 130.3, 128.9, 127.1, 124.5, 68.1, 29.0, 25.9 (M=CMePh), 24.9, 24.7, 24.4, 22.5.

Mo(NAr)(CMePh) $\left(\mathbf{O C}_{6} \mathbf{F}_{5}\right)_{2}(\mathbf{M e C N}) \quad(\mathbf{2}(\mathrm{MeCN}))$. Solid $\quad\left[\mathrm{Mo}(\mathrm{NAr})(\mathrm{CMePh})\left(\mathrm{OC}_{6} \mathrm{~F}_{5}\right)_{2}\right]_{2}$ $(0.007 \mathrm{~g}, 0.0094 \mathrm{mmol})$ was dissolved in $\mathrm{CD}_{3} \mathrm{CN}(0.6 \mathrm{~mL})$ in order to give an orange solution. Single crystals of $2(\mathrm{MeCN})$ suitable for XRD were obtained through addition of $\mathrm{MeCN}(0.5 \mathrm{~mL})$ to solid $2(0.020 \mathrm{~g})$ to produce an orange solution that was filtered through Celite and chilled at $30{ }^{\circ} \mathrm{C}$ for $12 \mathrm{~h}:{ }^{1} \mathrm{H}$ NMR $\left(600 \mathrm{MHz}, \mathrm{CD}_{3} \mathrm{CN}\right)$ Major Isomer: $\delta 7.4-7.3(\mathrm{~m}, 3 \mathrm{H}), 7.2(\mathrm{dd}, J=8.9$, $6.7 \mathrm{~Hz}, 2 \mathrm{H}), 7.2(\mathrm{~s}, 2 \mathrm{H}), 7.1(\mathrm{~d}, J=1.6 \mathrm{~Hz}, 1 \mathrm{H}), 3.7(\mathrm{~s}, 3 \mathrm{H}), 3.7$ (hept, $J=6.7 \mathrm{~Hz}, 2 \mathrm{H}), 1.0(\mathrm{~d}, J=$ $4.5 \mathrm{~Hz}, 6 \mathrm{H}), 1.0(\mathrm{~d}, J=4.6 \mathrm{~Hz}, 6 \mathrm{H}), 1.0(\mathrm{~s}, 3 \mathrm{H}) ;{ }^{19} \mathrm{~F}\left\{{ }^{1} \mathrm{H}\right\} \mathrm{NMR}\left(564 \mathrm{MHz}, \mathrm{CD}_{3} \mathrm{CN}\right) \delta-163.5(\mathrm{~d}$, $J=19.5 \mathrm{~Hz}),-164.2(\mathrm{~d}, J=19.5 \mathrm{~Hz}),-169.2(\mathrm{t}, J=20.4 \mathrm{~Hz}),-169.8(\mathrm{t}, J=20.7 \mathrm{~Hz}),-177.0(\mathrm{t}, J$ $=21.3 \mathrm{~Hz}),-177.7(\mathrm{t}, J=21.3 \mathrm{~Hz}) ;{ }^{13} \mathrm{C}\left\{{ }^{1} \mathrm{H}\right\} \mathrm{NMR}\left(151 \mathrm{MHz}, \mathrm{CD}_{3} \mathrm{CN}\right) \delta$ Selected resonances of major Isomer: $\delta 316.8,151.8,149.9,148.3,142.3(\mathrm{~m}), 140.7(\mathrm{~m}), 139.7(\mathrm{~m}), 138.0(\mathrm{~m}), 130.1$, 129.3, 128.9, 126.8, 124.2, 29.1, 28.7, 28.5, 25.3, 24.8, 24.4, 24.4, 23.6. Selected resonances of the minor isomer 1: ${ }^{1} \mathrm{H}$ NMR $\left(600 \mathrm{MHz}, \mathrm{CD}_{3} \mathrm{CN}\right) \delta 4.05$ (hept, $\left.J=6.5 \mathrm{~Hz}, 2 \mathrm{H}\right), 3.98(\mathrm{~s}, 3 \mathrm{H}), 0.99$ $(\mathrm{s}, 3 \mathrm{H}) ;{ }^{13} \mathrm{C}\left\{{ }^{1} \mathrm{H}\right\} \mathrm{NMR}\left(151 \mathrm{MHz}, \mathrm{CD}_{3} \mathrm{CN}\right) \delta 354.9,37.4$. Selected resonances of the minor isomer 2: ${ }^{1} \mathrm{H}$ NMR $\left(600 \mathrm{MHz}, \mathrm{CD}_{3} \mathrm{CN}\right) \delta 3.57$ (hept, $\left.J=6.5 \mathrm{~Hz}, 2 \mathrm{H}\right), 3.45(\mathrm{~s}, 3 \mathrm{H}), 0.98(\mathrm{~s}, 3 \mathrm{H}) ;{ }^{13} \mathrm{C}\left\{{ }^{1} \mathrm{H}\right\}$ NMR (151 MHz, CD $\left.{ }_{3} \mathrm{CN}\right) \delta 294.9,19.5$.

Note: Orange crystals of 2(MeCN) readily lose $\mathrm{MeCN}$ under vacuum (10-2 mbar, 10 minutes) and collapse to a yellow powder. For this reason, consistent elemental analyses for 2 (MeCN) could not be obtained.

Mo(NAr)(CMePh)( $\left(\mathbf{O C}_{6} \mathbf{F}_{5}\right)_{2}$ (bipy) (2(bipy)). A solution of 2, 2'-Bipyridine (0.004 g, 0.027 $\mathrm{mmol})$ in THF $(1 \mathrm{~mL})$ was added dropwise to a solution of $\left[\mathrm{Mo}(\mathrm{NAr})(\mathrm{CMePh})\left(\mathrm{OC}_{6} \mathrm{~F}_{5}\right)_{2}\right]_{2}(0.020$ $\mathrm{g}, 0.026 \mathrm{mmol})$ in THF $(1 \mathrm{~mL})$. The reaction was stirred for 30 minutes prior to removing volatiles in vacuo. The orange residue was dissolved in minimal $\mathrm{Et}_{2} \mathrm{O}(0.5 \mathrm{~mL})$. Pentane $(1 \mathrm{~mL})$ was added and the solution filtered rapidly through Celite. The filtrate was cooled to $-30{ }^{\circ} \mathrm{C}$ for $12 \mathrm{~h}$ during which time orange crystals formed. The product was filtered off, washed with pentane $(1 \mathrm{~mL})$, and dried in vacuo: yield $0.022 \mathrm{~g}(98 \%)$. A crystal suitable for XRD was obtained by vapor diffusion of $\mathrm{Et}_{2} \mathrm{O}$ into a concentrated dichloromethane solution: ${ }^{1} \mathrm{H}$ NMR $\left(600 \mathrm{MHz}, \mathrm{C}_{6} \mathrm{D}_{6}\right) \delta 9.52$ $(\mathrm{d}, J=5.0 \mathrm{~Hz}, 1 \mathrm{H}), 8.57(\mathrm{~d}, J=5.4 \mathrm{~Hz}, 1 \mathrm{H}), 7.60(\mathrm{~d}, J=7.8 \mathrm{~Hz}, 2 \mathrm{H}), 7.13(\mathrm{t}, J=7.8 \mathrm{~Hz}, 2 \mathrm{H})$, $6.94-6.84(\mathrm{~m}, 1 \mathrm{H}), 6.85(\mathrm{t}, J=7.3 \mathrm{~Hz}, 1 \mathrm{H}), 6.74(\mathrm{~m}, J=7.7 \mathrm{~Hz}, 5 \mathrm{H}), 6.62(\mathrm{~d}, J=14.6,8.3 \mathrm{~Hz}$, $1 \mathrm{H}), 6.57-6.53(\mathrm{~m}, 1 \mathrm{H}), 6.42(\mathrm{td}, J=6.4,5.7,1.1 \mathrm{~Hz}, 1 \mathrm{H}), 4.70(\mathrm{~s}, 1 \mathrm{H}), 4.15(\mathrm{~s}, 3 \mathrm{H}), 2.68(\mathrm{~s}$, $1 \mathrm{H}), 1.44-1.31(\mathrm{~m}, 3 \mathrm{H}), 1.26(\mathrm{~d}, J=7.0 \mathrm{~Hz}, 3 \mathrm{H}), 0.56-0.42(\mathrm{~m}, 3 \mathrm{H}),-0.09--0.22(\mathrm{~m}, 3 \mathrm{H})$; ${ }^{19} \mathrm{~F}\left\{{ }^{1} \mathrm{H}\right\}$ NMR $\left(564 \mathrm{MHz}, \mathrm{C}_{6} \mathrm{D}_{6}\right) \delta-160.63(\mathrm{dt}, J=17.6,8.6 \mathrm{~Hz}),-160.76(\mathrm{dq}, J=16.9,7.7 \mathrm{~Hz})$, $-168.16(\mathrm{t}, J=21.5 \mathrm{~Hz}),-168.94(\mathrm{t}, J=21.5 \mathrm{~Hz}),-176.16(\mathrm{tt}, J=23.5,7.7 \mathrm{~Hz}),-178.04(\mathrm{tt}, J=$ 22.3, 8.0 Hz); ${ }^{13} \mathrm{C}\left\{{ }^{1} \mathrm{H}\right\}$ NMR $\left(151 \mathrm{MHz}, \mathrm{C}_{6} \mathrm{D}_{6}\right) \delta 316.9,158.0,157.9,153.9,152.5,150.4,150.0$, 149.6, 147.9, 143.1, 142.4, 141.5, 140.8, 140.0, 139.8, 138.9, 138.1, 137.2, 134.1 (t, $J=15.0 \mathrm{~Hz}$ ), 132.5 (q, $J=16.7,15.2 \mathrm{~Hz}), 131.0(\mathrm{t}, J=13.2 \mathrm{~Hz}), 128.4,127.6,127.3,127.1,126.1,126.0,125.8$, $123.8,122.4,121.4,120.5,34.5,28.0,27.7,25.1,24.3,22.3$. Anal. Calcd. for $\mathrm{C}_{42} \mathrm{H}_{33} \mathrm{~F}_{10} \mathrm{MoN}_{3} \mathrm{O}_{2}$ $\left(+1 \mathrm{C}_{4} \mathrm{H}_{8} \mathrm{O}\right): \mathrm{C}, 56.97 ; \mathrm{H}, 4.26 ; \mathrm{N}, 4.33$. Found: $\mathrm{C}, 57.22 ; \mathrm{H}, 4.16 ; \mathrm{N}, 4.30$. 
Mo(NAr)(CMePh)(Cl)2(bipy) (3(bipy)). Chlorotrimethylsilane $(0.091 \mathrm{~g}, 0.838 \mathrm{mmol})$ was added to a stirred suspension of $\mathrm{Mo}(\mathrm{NAr})(\mathrm{CMePh})\left(\mathrm{OC}_{6} \mathrm{~F}_{5}\right)_{2}$ (bipy) $(0.188 \mathrm{~g}, 0.209 \mathrm{mmol})$ in $\mathrm{Et}_{2} \mathrm{O}$ $(6 \mathrm{~mL})$. The reaction was stirred for $12 \mathrm{~h}$ during which time an orange solid precipitated. The product was collected on a frit, washed with $\mathrm{Et}_{2} \mathrm{O}(4 \mathrm{x} 1 \mathrm{~mL})$ and dried in vacuo: yield $0.112 \mathrm{~g}$ $(89 \%)$. A crystal suitable for XRD was obtained through layering a concentrated dichloromethane solution with $\mathrm{Et}_{2} \mathrm{O}:{ }^{1} \mathrm{H} \mathrm{NMR}\left(600 \mathrm{MHz}, \mathrm{CD}_{2} \mathrm{Cl}_{2},-20^{\circ} \mathrm{C}\right) \delta 9.4(\mathrm{~d}, J=4.2 \mathrm{~Hz}, 1 \mathrm{H}), 8.8(\mathrm{~d}, J=4.6$ $\mathrm{Hz}, 1 \mathrm{H}), 8.3(\mathrm{~d}, J=8.0 \mathrm{~Hz}, 1 \mathrm{H}), 8.2(\mathrm{~d}, J=8.0 \mathrm{~Hz}, 2 \mathrm{H}), 8.1-8.0(\mathrm{~m}, 1 \mathrm{H}), 7.7-7.7(\mathrm{~m}, 1 \mathrm{H}), 7.7$ $-7.6(\mathrm{~m}, 1 \mathrm{H}), 7.3(\mathrm{~d}, J=7.3 \mathrm{~Hz}, 2 \mathrm{H}), 7.1(\mathrm{t}, J=7.8 \mathrm{~Hz}, 2 \mathrm{H}), 6.9(\mathrm{dt}, J=13.0,7.1 \mathrm{~Hz}, 4 \mathrm{H}), 6.6$ $(\mathrm{d}, J=7.1 \mathrm{~Hz}, 1 \mathrm{H}), 4.5(\mathrm{dt}, J=13.2,6.1 \mathrm{~Hz}, 1 \mathrm{H}), 4.1(\mathrm{~s}, 2 \mathrm{H}), 2.7(\mathrm{dd}, J=13.0,6.2 \mathrm{~Hz}, 1 \mathrm{H}), 1.2$ $(\mathrm{d}, J=6.3 \mathrm{~Hz}, 3 \mathrm{H}), 1.0(\mathrm{~d}, J=6.4 \mathrm{~Hz}, 3 \mathrm{H}), 0.3(\mathrm{~d}, J=6.4 \mathrm{~Hz}, 3 \mathrm{H}),-0.1(\mathrm{~d}, J=6.4 \mathrm{~Hz}, 3 \mathrm{H})$; ${ }^{13} \mathrm{C}\left\{{ }^{1} \mathrm{H}\right\}$ NMR $\left(151 \mathrm{MHz}, \mathrm{CD}_{2} \mathrm{Cl}_{2},-20{ }^{\circ} \mathrm{C}\right) \delta 324.2,159.2,157.2,154.4,152.5,151.7,149.2$, $148.9,148.4,141.0,139.7,128.6,127.8,127.5,127.3,127.0,126.7,125.4,123.8,123.5,122.9$, 122.5, 38.9, 27.9, 27.5, 25.0, 24.6, 24.1, 22.1. Anal. Calcd. for $\mathrm{C}_{30} \mathrm{H}_{33} \mathrm{Cl}_{2} \mathrm{MoN}_{3}\left(+1 \mathrm{CH}_{2} \mathrm{Cl}_{2}\right): \mathrm{C}$, 54.17; H, 5.13; N, 6.11. Found: C, 54.10; H, 5.21; N, 5.72.

Mo(NAr)(CMePh)(Cl)(OHMT) (4). A stirred suspension of $\mathrm{Mo}(\mathrm{NAr})(\mathrm{CMePh})(\mathrm{Cl})_{2}$ (bipy) $(0.121 \mathrm{~g}, 0.202 \mathrm{mmol})$ in $\mathrm{Et}_{2} \mathrm{O}(5 \mathrm{~mL})$ was cooled to $-30{ }^{\circ} \mathrm{C}$ before adding a $-30{ }^{\circ} \mathrm{C}$ solution of LiOHMT (0.081 g, $0.242 \mathrm{mmol})$ and $\mathrm{ZnCl}_{2} \bullet$ dioxane $(0.047 \mathrm{~g}, 0.209 \mathrm{mmol})$ in THF (3 mL). The suspension was stirred for 12 hours at $22^{\circ} \mathrm{C}$ to yield a ruby red solution and a colorless precipitate. All volatiles were removed from the mixture in vacuo, and the residue was extracted with pentane $(3 \mathrm{~mL})$. The extract was filtered through Celite and volatiles were removed in vacuo to afford a ruby red oil. The oil was dissolved in benzene $(0.5 \mathrm{~mL})$ and lyophilized to yield a red foam that aided manipulation: yield $0.307 \mathrm{~g}(73 \%)$. Anal. Calcd. for $\mathrm{C}_{44} \mathrm{H}_{50} \mathrm{ClMoNO}$ : C, 71.39; H, 6.81; N, 1.89. Found: C, 70.96; H, 6.44; N, 1.67.

Mo(NAr)(CMePh)(Cl)(OHMT)(MeCN) (4(MeCN)). Mo(NAr)(CMePh)(Cl)(OHMT) $(0.010 \mathrm{~g}, 0.014 \mathrm{mmol})$ was added to $\mathrm{CD}_{3} \mathrm{CN}(0.5 \mathrm{~mL})$ to produce a yellow solution. A crystal suitable for XRD was obtained by cooling a concentrated solution of a toluene:MeCN:pentane (1:1:2) mixture at $-30{ }^{\circ} \mathrm{C}$ for $24 \mathrm{~h}:{ }^{1} \mathrm{H}$ NMR $\left(600 \mathrm{MHz}, \mathrm{C}_{6} \mathrm{D}_{6}\right) \delta 7.1(\mathrm{~d}, J=7.7 \mathrm{~Hz}, 2 \mathrm{H}), 7.0-6.9$ $(\mathrm{m}, 10 \mathrm{H}), 6.8(\mathrm{~s}, 2 \mathrm{H}), 6.7(\mathrm{tt}, J=14.7,2.4 \mathrm{~Hz}, 1 \mathrm{H}), 3.7$ (hept, $J=6.3,5.7 \mathrm{~Hz}, 2 \mathrm{H}), 3.0(\mathrm{~s}, 3 \mathrm{H}), 2.5$ $(\mathrm{s}, 6 \mathrm{H}), 2.4(\mathrm{~s}, 6 \mathrm{H}), 2.3(\mathrm{~s}, 6 \mathrm{H}), 1.3(\mathrm{~d}, J=6.8 \mathrm{~Hz}, 6 \mathrm{H}), 1.2(\mathrm{~d}, J=6.7 \mathrm{~Hz}, 6 \mathrm{H}), 0.6(\mathrm{~s}, 3 \mathrm{H}) ;{ }^{13} \mathrm{C}\left\{{ }^{1} \mathrm{H}\right\}$ NMR $\left(151 \mathrm{MHz}, \mathrm{C}_{6} \mathrm{D}_{6}\right) \delta 296.8,160.1,152.9,148.6,146.8,137.2,137.1,136.8,136.2,131.8$, $129.8,128.9,128.5,128.4,127.8,126.1,123.3,121.7,118.4,38.9,28.5,24.0,23.9,22.1,21.2$, 21.2, 21.0, 0.4. ${ }^{1} \mathrm{H}$ NMR (600 MHz, $\left.\mathrm{CD}_{3} \mathrm{CN}\right)$ Major Isomer: $\delta 7.2-7.2(\mathrm{~m}, 2 \mathrm{H}), 7.1-7.0(\mathrm{~s}, 5 \mathrm{H})$, $7.0-7.0(\mathrm{~m}, 1 \mathrm{H}), 7.0-7.0(\mathrm{~s}, 1 \mathrm{H}), 7.0-6.9(\mathrm{~m}, 2 \mathrm{H}), 6.9-6.8(\mathrm{~m}, 2 \mathrm{H}), 6.8(\mathrm{~s}, 1 \mathrm{H}), 6.8(\mathrm{~s}, 1 \mathrm{H})$, $3.4(\mathrm{~s}, 1 \mathrm{H}), 3.2(\mathrm{dq}, J=13.4,6.8 \mathrm{~Hz}, 1 \mathrm{H}), 2.6(\mathrm{~s}, 3 \mathrm{H}), 2.2(\mathrm{~s}, 6 \mathrm{H}), 2.2(\mathrm{~s}, 6 \mathrm{H}), 2.1(\mathrm{~s}, 3 \mathrm{H}), 2.0(\mathrm{~s}$, $3 \mathrm{H}), 1.9(\mathrm{~s}, 3 \mathrm{H}), 1.0(\mathrm{~d}, J=5.6 \mathrm{~Hz}, 6 \mathrm{H}), 0.9(\mathrm{~d}, J=6.8 \mathrm{~Hz}, 3 \mathrm{H}), 0.9(\mathrm{~d}, J=6.8 \mathrm{~Hz}, 3 \mathrm{H}) ;{ }^{13} \mathrm{C}\left\{{ }^{1} \mathrm{H}\right\}$ NMR (151 MHz, $\left.\mathrm{CD}_{3} \mathrm{CN}\right)$ Major Isomer: $\delta 323.6,161.7,152.0,150.5,138.0,137.8,137.5,137.4$, $137.1,137.0,131.4,131.3,130.4,130.2$, 129.8, 129.6, 129.4, 129.0, 128.7, 128.6, 128.4, 128.2, $128.1,127.1,126.9,126.1,125.7,123.7,121.1,28.4,28.3,27.7,26.9,24.9,23.6,23.1,21.6,21.3$, 21.1, 21.1, 21.0, 20.7, 20.4. Selected resonances of the minor isomer: ${ }^{1} \mathrm{H} N \mathrm{NR}\left(600 \mathrm{MHz}, \mathrm{CD}_{3} \mathrm{CN}\right)$ $\delta 6.8$ (s), 6.7 (s), 2.8 (s), 2.3 (s), 2.3 (s), $1.0(\mathrm{bs}) ;{ }^{13} \mathrm{C}\left\{{ }^{1} \mathrm{H}\right\} \mathrm{NMR}\left(151 \mathrm{MHz}, \mathrm{CD}_{3} \mathrm{CN}\right)$ 160.8, 151.2, 147.6, 135.1, 129.1, 25.0, 23.0.

Note: Yellow crystals of 4(MeCN) readily lose MeCN under vacuum (10-2 mbar, 10 minutes) and turn red. For this reason elemental analysis for $4(\mathrm{MeCN})$ could not be obtained. 
Mo(NAr)(CMePh)(OHMT) $\left(\mathrm{NC}_{4} \mathbf{H}_{4}\right)$ (5). A chilled $\left(\begin{array}{lll}-30 & \left.{ }^{\circ} \mathrm{C}\right) & \text { solution of }\end{array}\right.$ $\mathrm{Mo}(\mathrm{NAr})(\mathrm{CMePh})(\mathrm{Cl})(\mathrm{OHMT})(0.100 \mathrm{~g}, 0.135 \mathrm{mmol})$ in $\mathrm{Et}_{2} \mathrm{O}(4 \mathrm{~mL})$ was added to a suspension of sodium pyrrolide $(0.013 \mathrm{~g}, 0.135 \mathrm{mmol})$ in $\mathrm{Et}_{2} \mathrm{O}(3 \mathrm{~mL})$ that had been cooled to $-30{ }^{\circ} \mathrm{C}$. The reaction was stirred 2 hours during which time an orange/red solution was obtained along with a precipitate. Volatiles were removed in vacuo and the residue was extracted into pentane $(3 \mathrm{x} 1$ $\mathrm{mL}$ ). The extract was filtered through Celite and the volatiles removed from the filtrate in vacuo to afford the product as an orange/red foam: yield $0.060 \mathrm{~g}(72 \%)$. A crystal suitable for XRD was obtained through slow evaporation of a concentrated heptane solution into silicon grease at -30 ${ }^{\circ} \mathrm{C}:{ }^{1} \mathrm{H}$ NMR $\left(600 \mathrm{MHz}, \mathrm{C}_{6} \mathrm{D}_{6}\right) \delta 7.4(\mathrm{~d}, J=7.4 \mathrm{~Hz}, 2 \mathrm{H}), 7.2-7.0(\mathrm{~m}, 10 \mathrm{H}), 7.0(\mathrm{~s}, 2 \mathrm{H}), 6.9(\mathrm{t}, J$ $=7.3 \mathrm{~Hz}, 1 \mathrm{H}), 6.57(\mathrm{t}, J=1.7 \mathrm{~Hz}, 2 \mathrm{H}), 6.40(\mathrm{t}, J=1.7 \mathrm{~Hz}, 2 \mathrm{H}), 3.3$ (hept, $J=6.6 \mathrm{~Hz}, 2 \mathrm{H}), 2.7(\mathrm{~s}$, $3 \mathrm{H}), 2.5(\mathrm{~s}, 6 \mathrm{H}), 2.3(\mathrm{~s}, 6 \mathrm{H}), 2.2(\mathrm{~s}, 6 \mathrm{H}), 1.1(\mathrm{~d}, J=6.8 \mathrm{~Hz}, 6 \mathrm{H}), 1.0(\mathrm{~d}, J=6.7 \mathrm{~Hz}, 6 \mathrm{H}) ;{ }^{13} \mathrm{C}\left\{{ }^{1} \mathrm{H}\right\}$ NMR $\left(151 \mathrm{MHz}, \mathrm{C}_{6} \mathrm{D}_{6}\right) \delta 290.6,159.2,152.6,148.5,146.7,137.4,137.1,136.9,136.4,132.1$, 130.0, 128.8, 128.8, 128.6, 127.7, 126.8, 123.2, 122.7, 110.6, 28.7, 24.0, 23.5, 21.2, 20.9, 20.6, 20.2. Anal. Calcd. for $\mathrm{C}_{48} \mathrm{H}_{54} \mathrm{MoN}_{2} \mathrm{O}$ : C, 74.78; H, 7.06; N, 3.63. Found: C, 74.60; H, 7.05; N, 3.62 .

\section{Spectroscopic Data}

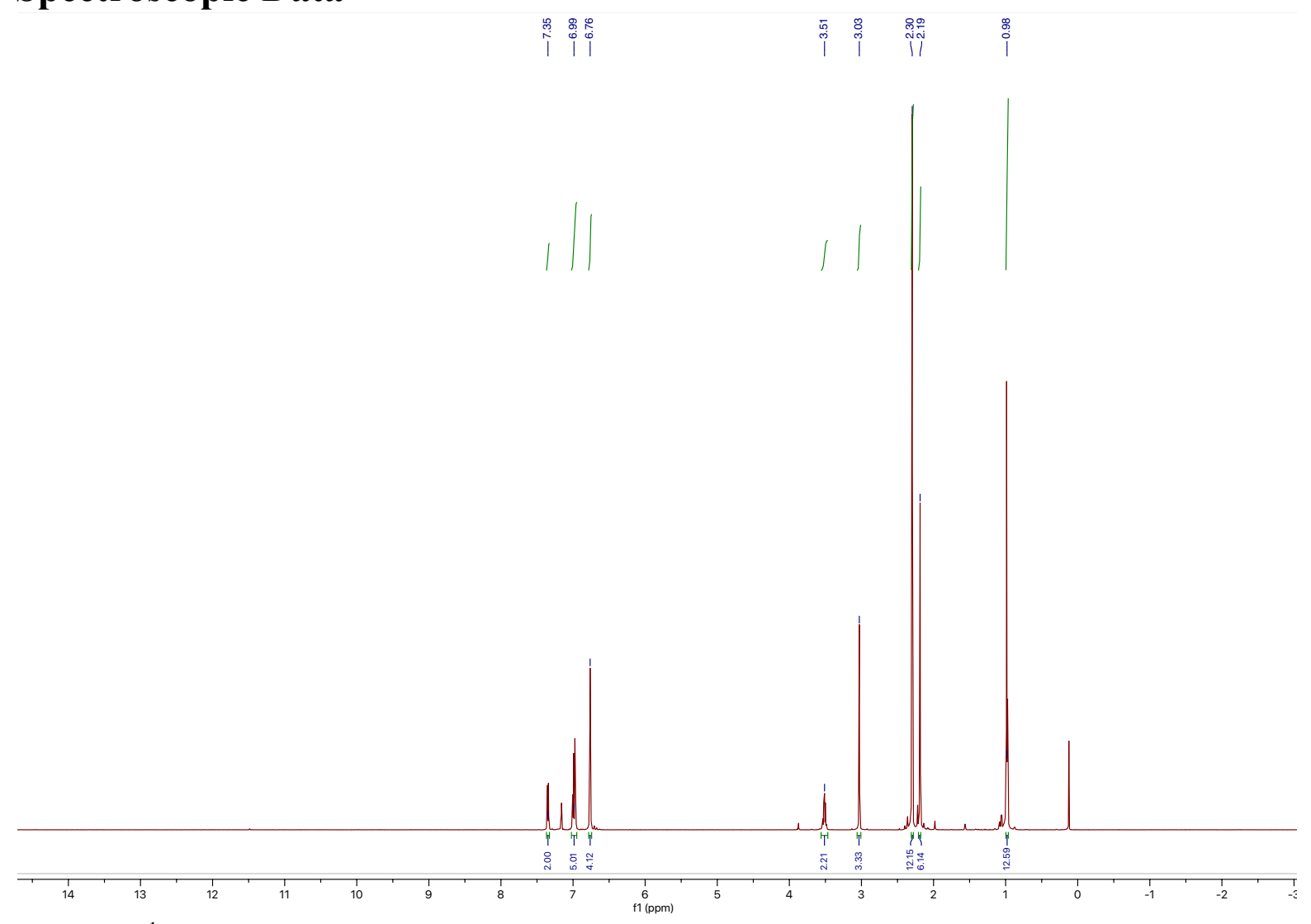

Figure S1. ${ }^{1} \mathrm{H}$ NMR spectrum of $\mathrm{Mo}(\mathrm{NAr})(\mathrm{CMePh})(\mathrm{OMes})_{2}$ recorded at $600 \mathrm{MHz}$ in $\mathrm{C}_{6} \mathrm{D}_{6}$. 


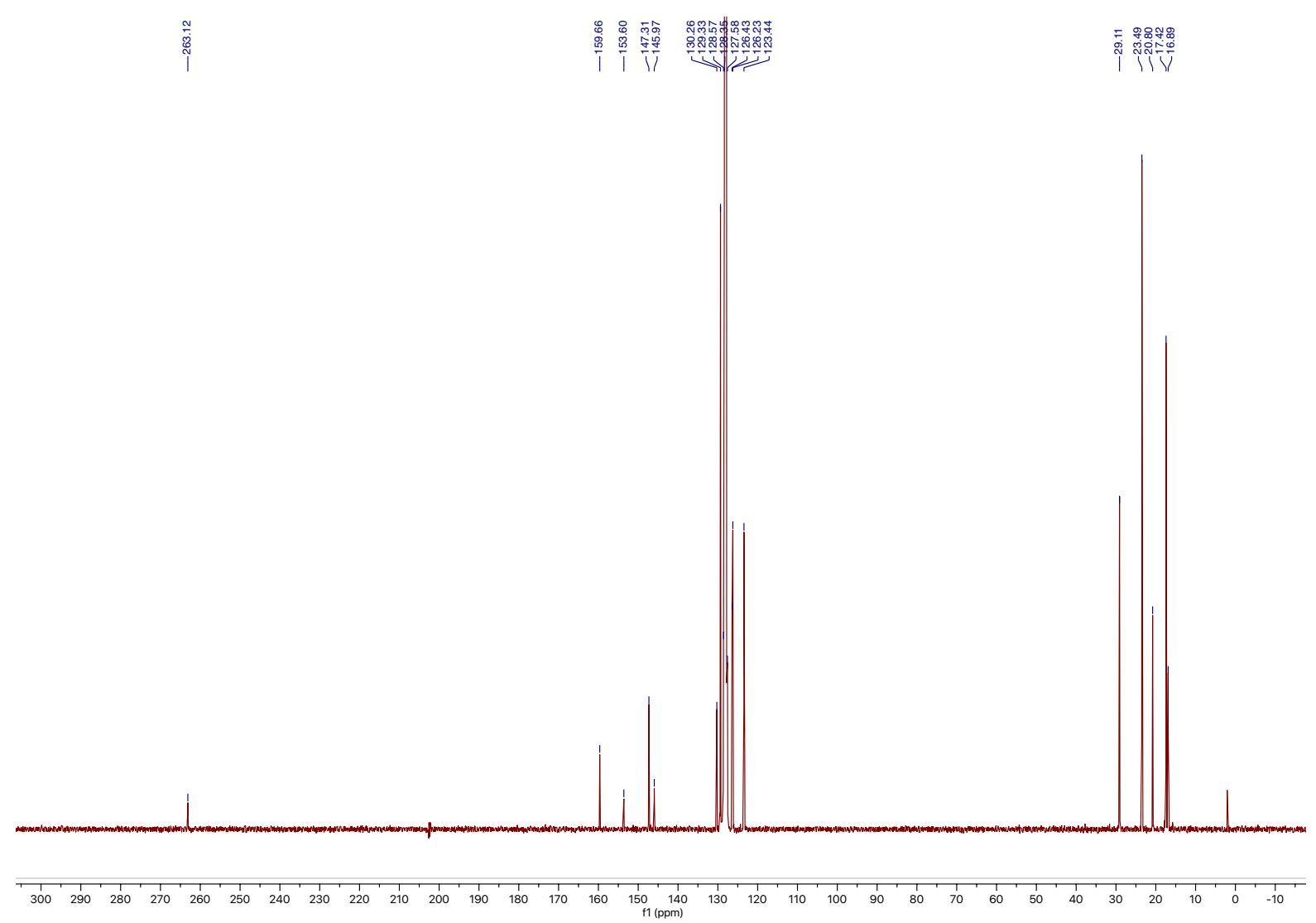

Figure S2. ${ }^{13} \mathrm{C}\left\{{ }^{1} \mathrm{H}\right\}$ NMR spectrum of $\mathrm{Mo}(\mathrm{NAr})(\mathrm{CMePh})(\mathrm{OMes})_{2}$ recorded at $151 \mathrm{MHz}$ in $\mathrm{C}_{6} \mathrm{D}_{6}$.

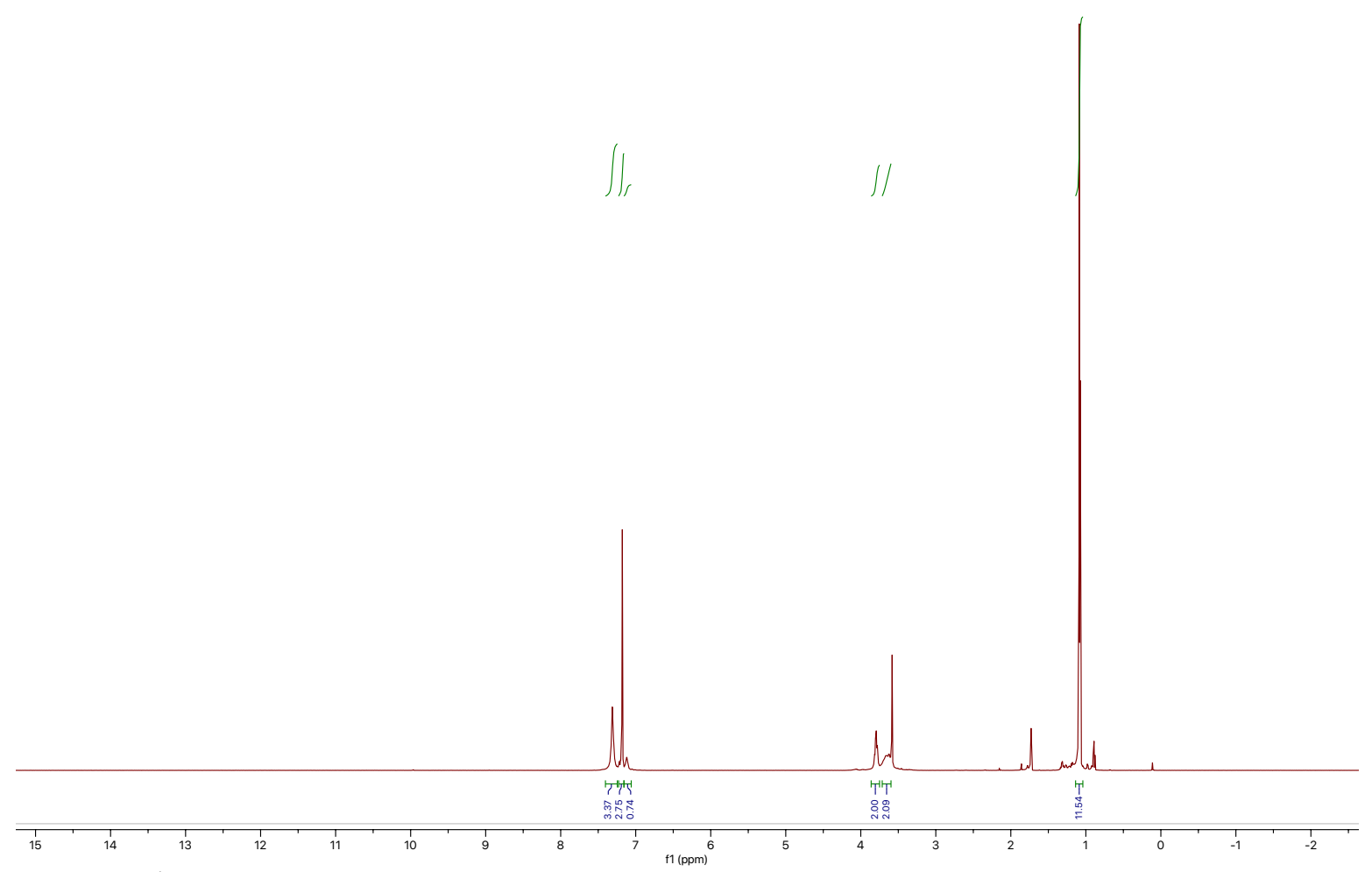

Figure S3. ${ }^{1} \mathrm{H}$ NMR spectrum of $\mathrm{Mo}(\mathrm{NAr})(\mathrm{CMePh})\left(\mathrm{OC}_{6} \mathrm{~F}_{5}\right)_{2}(\mathrm{THF})_{\mathrm{x}}$ recorded at $600 \mathrm{MHz}$ in $\mathrm{THF}-d_{8}$ at $22{ }^{\circ} \mathrm{C}$. 


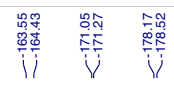
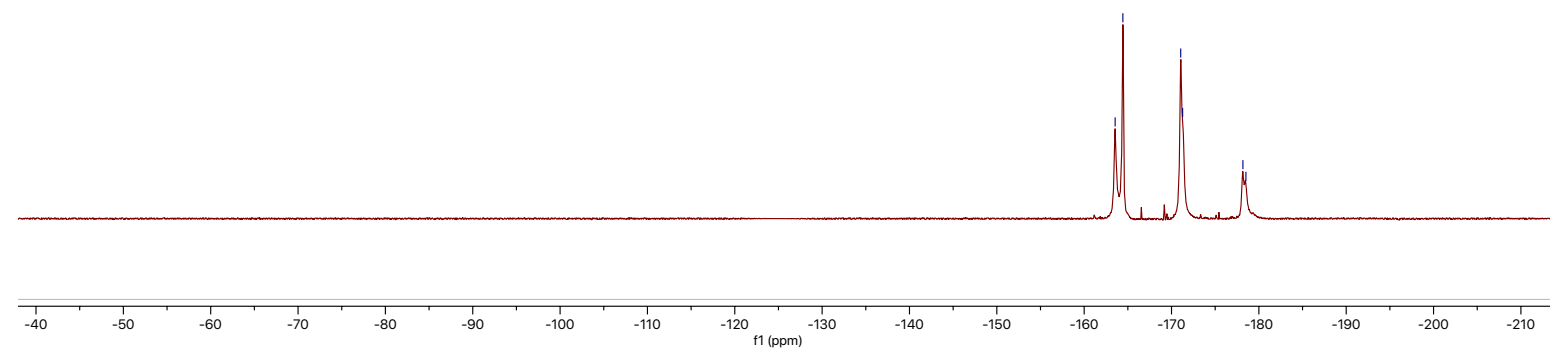

Figure S4. ${ }^{19} \mathrm{~F}\left\{{ }^{1} \mathrm{H}\right\}$ NMR spectrum of $\mathrm{Mo}(\mathrm{NAr})(\mathrm{CMePh})\left(\mathrm{OC}_{6} \mathrm{~F}_{5}\right)_{2}(\mathrm{THF})_{\mathrm{x}}$ recorded at $565 \mathrm{MHz}$ in THF- $d_{8}$ at $22{ }^{\circ} \mathrm{C}$.
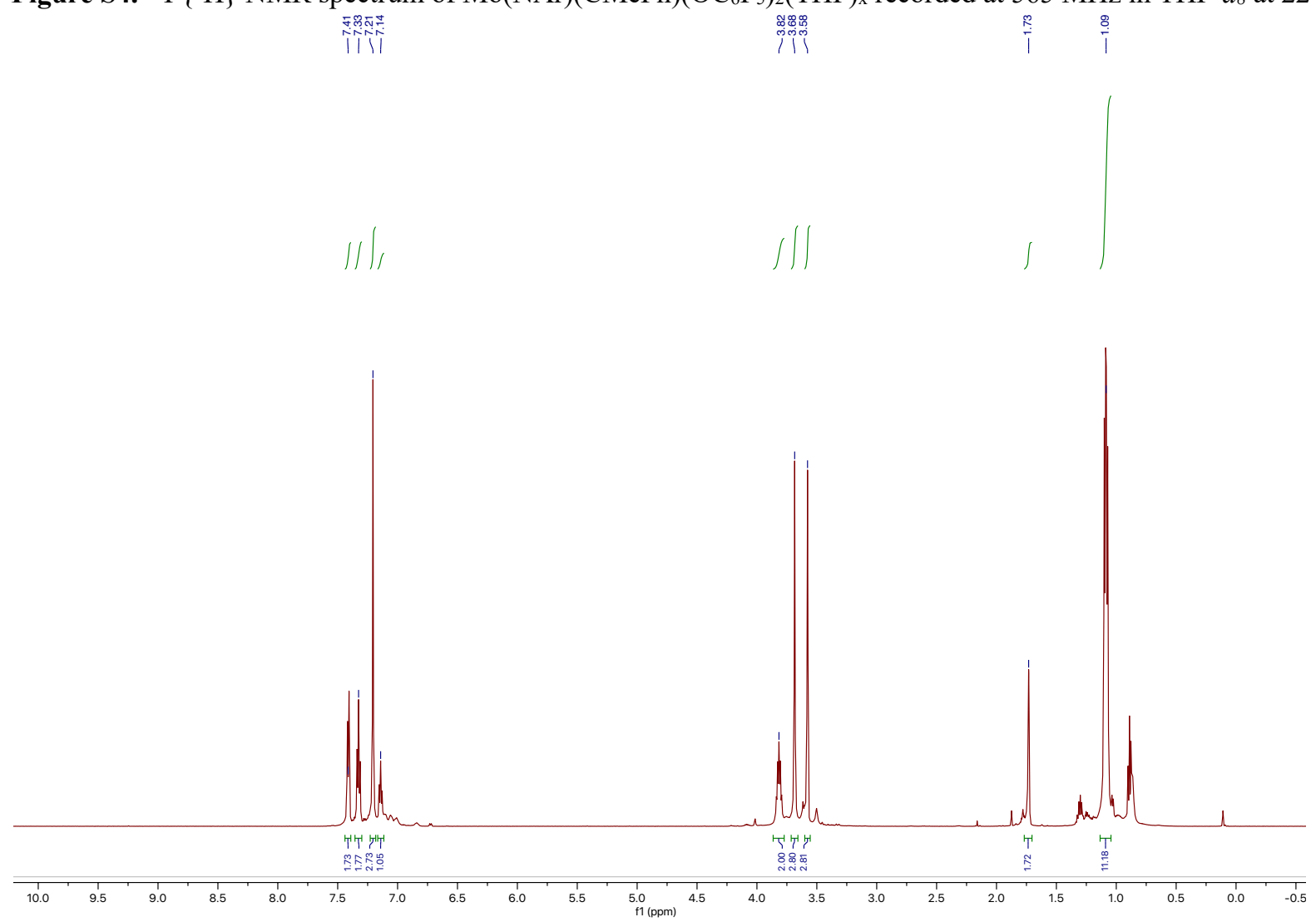

Figure S5. ${ }^{1} \mathrm{H}$ NMR spectrum of $\mathrm{Mo}(\mathrm{NAr})(\mathrm{CMePh})\left(\mathrm{OC}_{6} \mathrm{~F}_{5}\right)_{2}(\mathrm{THF})_{\mathrm{x}}$ recorded at $600 \mathrm{MHz}$ in THF- $d_{8}$ at $-40{ }^{\circ} \mathrm{C}$. 

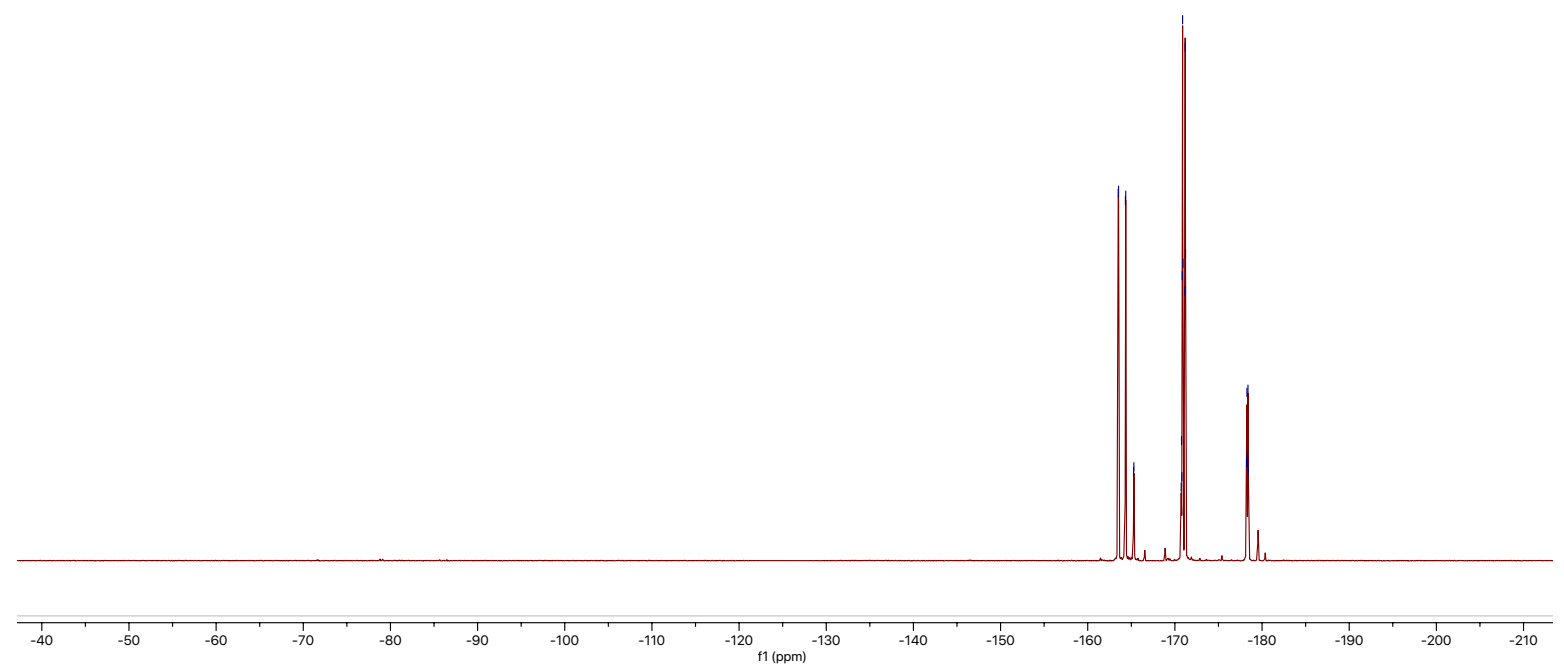

Figure S6. ${ }^{19} \mathrm{~F}\left\{{ }^{1} \mathrm{H}\right\}$ NMR spectrum of $\mathrm{Mo}(\mathrm{NAr})(\mathrm{CMePh})\left(\mathrm{OC}_{6} \mathrm{~F}_{5}\right)_{2}(\mathrm{THF})_{\mathrm{x}}$ recorded at $565 \mathrm{MHz}$ in THF- $d_{8}$ at $-40{ }^{\circ} \mathrm{C}$.

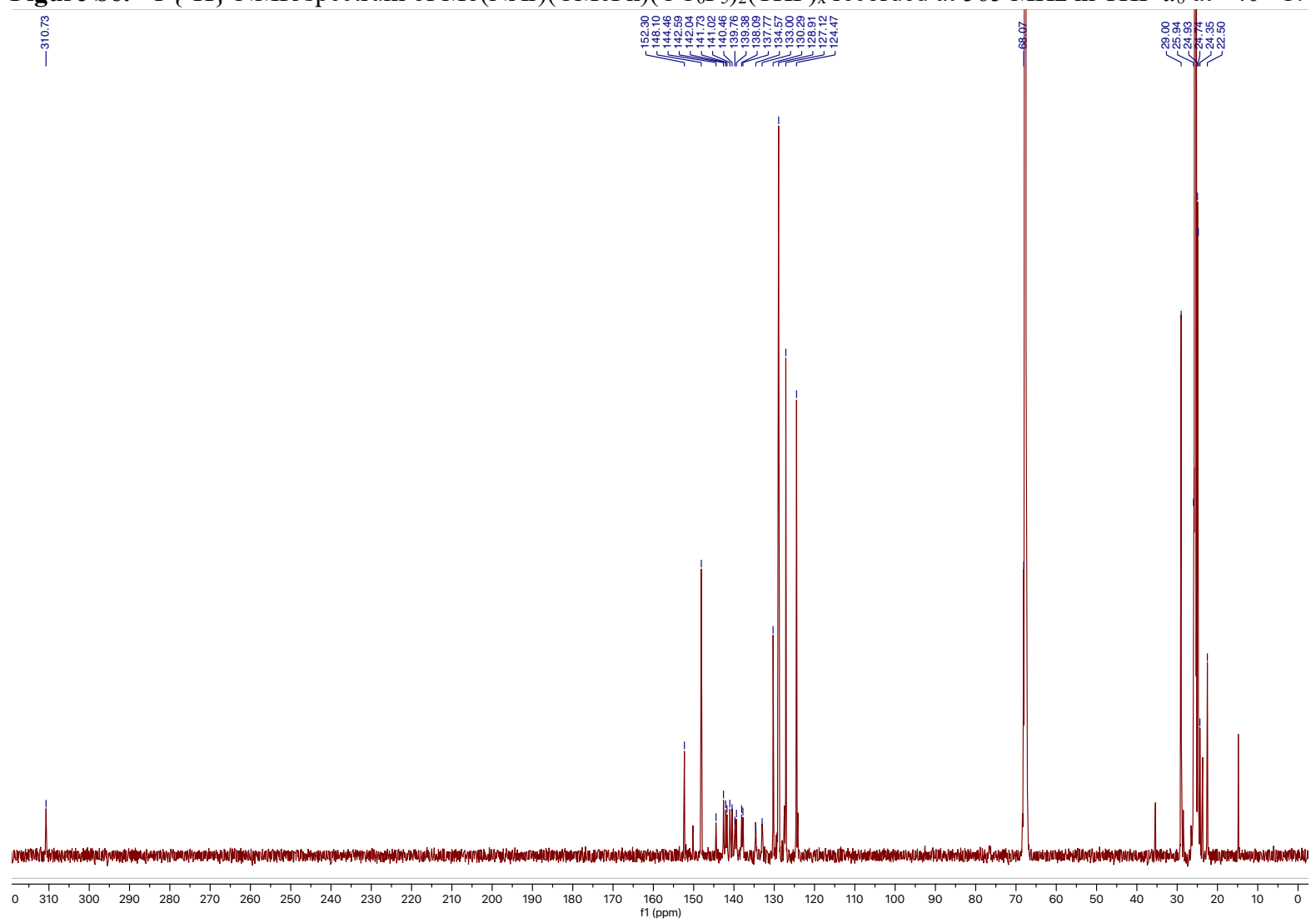

Figure S7. ${ }^{13} \mathrm{C}\left\{{ }^{1} \mathrm{H}\right\}$ NMR spectrum of $\mathrm{Mo}(\mathrm{NAr})(\mathrm{CMePh})\left(\mathrm{OC}_{6} \mathrm{~F}_{5}\right)_{2}(\mathrm{THF})_{\mathrm{x}}$ recorded at $151 \mathrm{MHz}$ in THF- $d_{8}$ at $-40{ }^{\circ} \mathrm{C}$. 


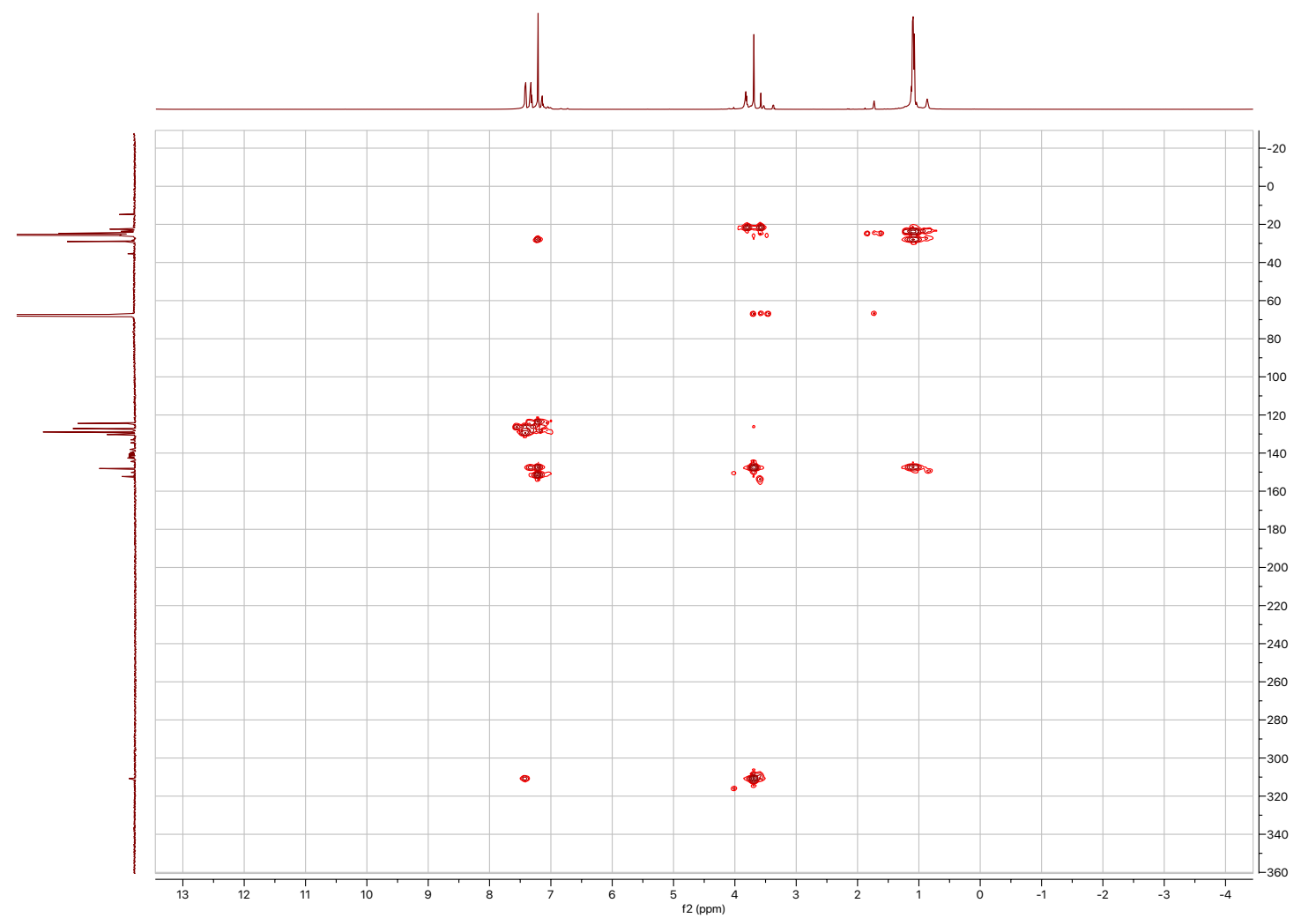

Figure S8. ${ }^{13} \mathrm{C},{ }^{1} \mathrm{H}-\mathrm{HMBC}$ NMR spectrum of $\mathrm{Mo}(\mathrm{NAr})(\mathrm{CMePh})\left(\mathrm{OC}_{6} \mathrm{~F}_{5}\right)_{2}(\mathrm{THF})_{\mathrm{x}}$ recorded at $600 \mathrm{MHz}$ in THF- $d_{8}$ at $-40{ }^{\circ} \mathrm{C}$.

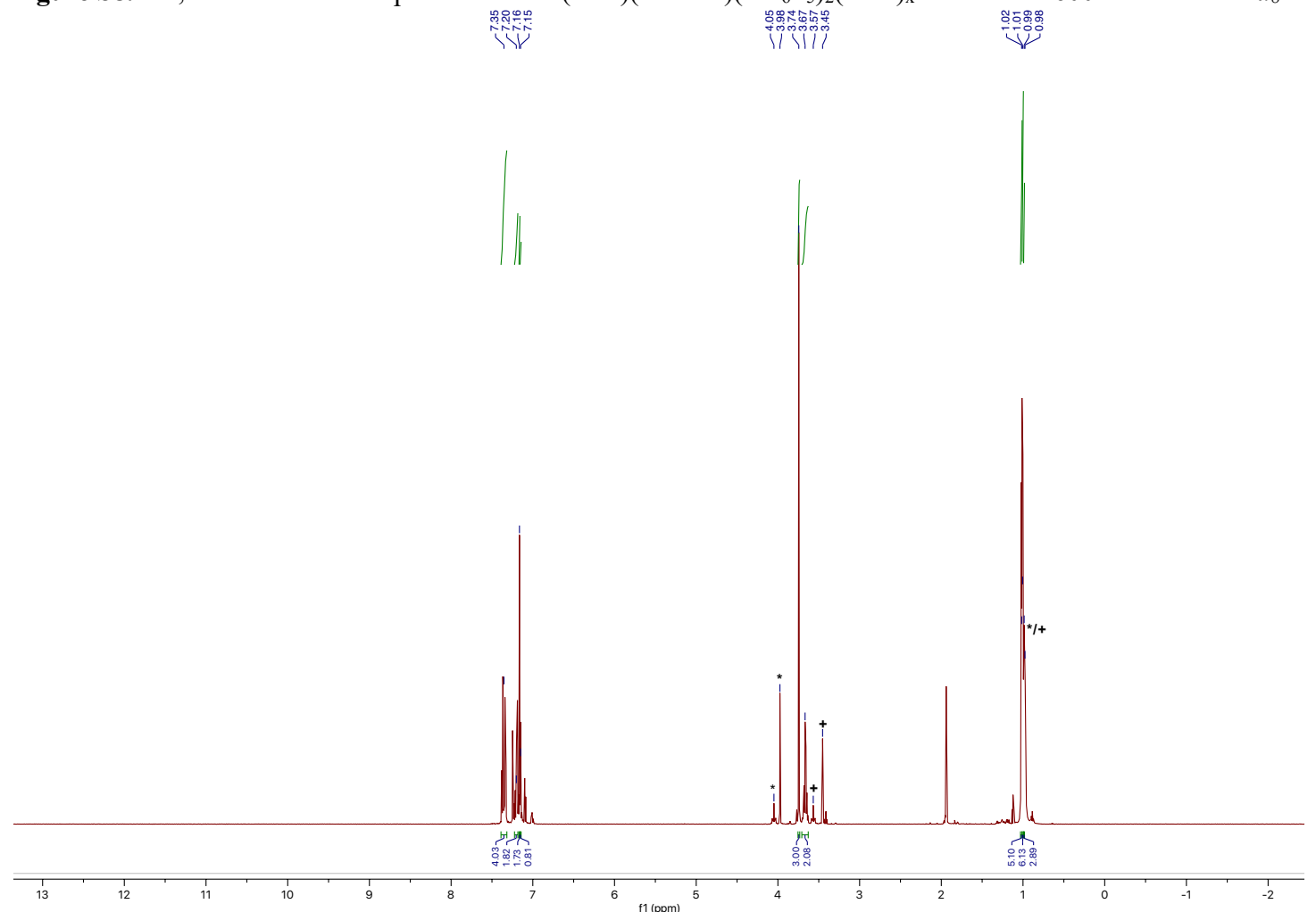

Figure S9. ${ }^{1} \mathrm{H}$ NMR spectrum of $\mathrm{Mo}(\mathrm{NAr})(\mathrm{CMePh})\left(\mathrm{OC}_{6} \mathrm{~F}_{5}\right)_{2}(\mathrm{MeCN})$ recorded at $600 \mathrm{MHz}$ in $\mathrm{CD}_{3} \mathrm{CN}$. The asterisk $(*)$ and plus $(+)$ symbols denote resonances for isomers 1 and 2, respectively, which are proposed to be $\mathrm{Mo}(\mathrm{NAr})(\mathrm{CMePh})\left(\mathrm{OC}_{6} \mathrm{~F}_{5}\right)_{2}(\mathrm{MeCN})_{2}$ complexes. 


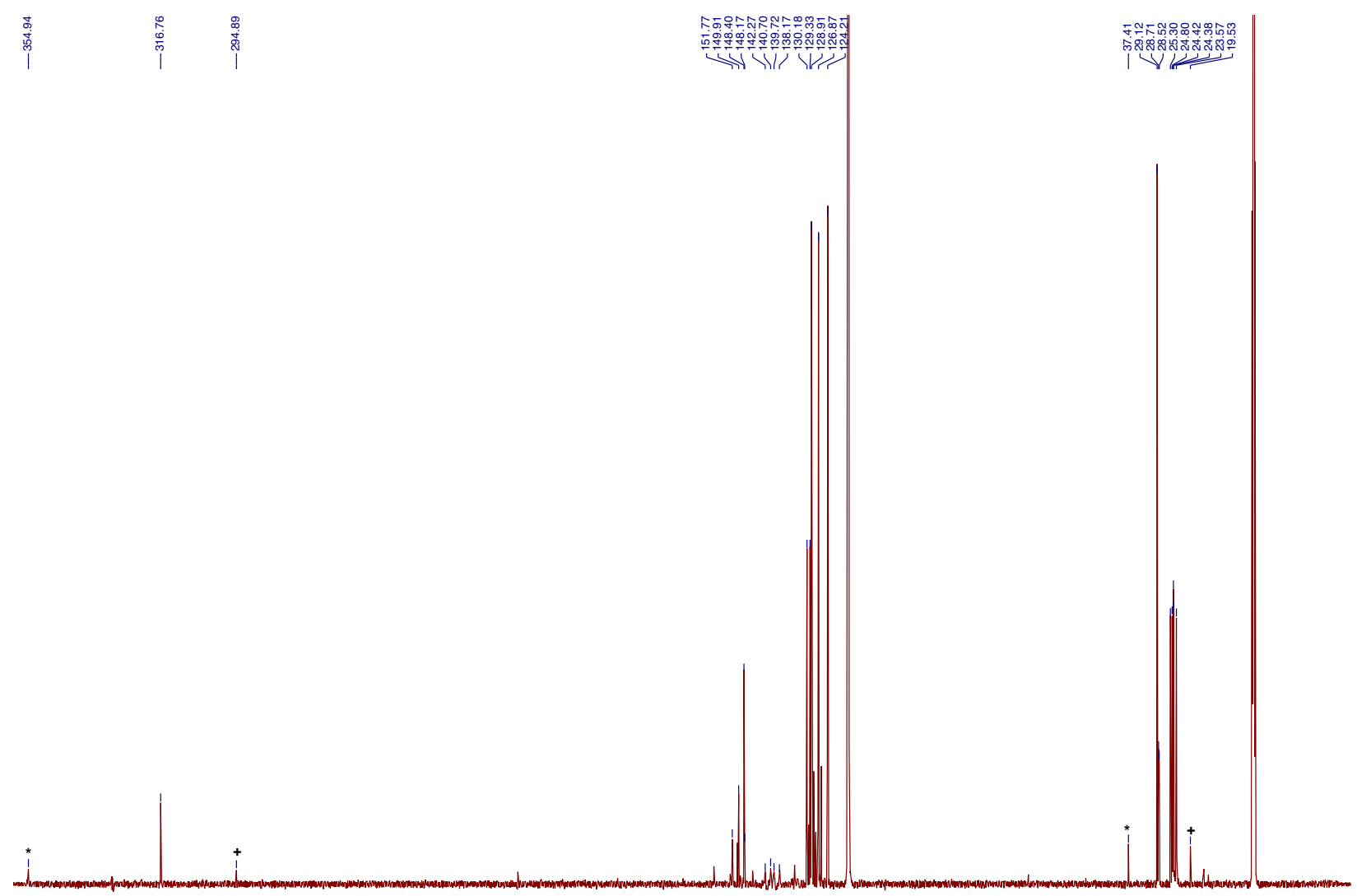

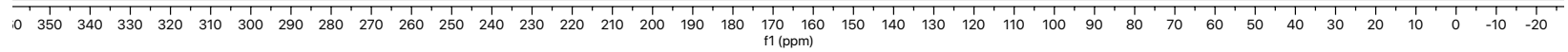

Figure S10. ${ }^{13} \mathrm{C}\left\{{ }^{1} \mathrm{H}\right\}$ NMR spectrum of $\mathrm{Mo}(\mathrm{NAr})(\mathrm{CMePh})\left(\mathrm{OC}_{6} \mathrm{~F}_{5}\right)_{2}(\mathrm{MeCN})$ recorded at $151 \mathrm{MHz}$ in $\mathrm{CD}_{3} \mathrm{CN}$. The asterisk $\left({ }^{*}\right)$ and plus $(+)$ symbols denotes resonances for isomers 1 and 2, respectively, which are proposed to be $\mathrm{Mo}(\mathrm{NAr})(\mathrm{CMePh})\left(\mathrm{OC}_{6} \mathrm{~F}_{5}\right)_{2}(\mathrm{MeCN})_{2}$ complexes. 


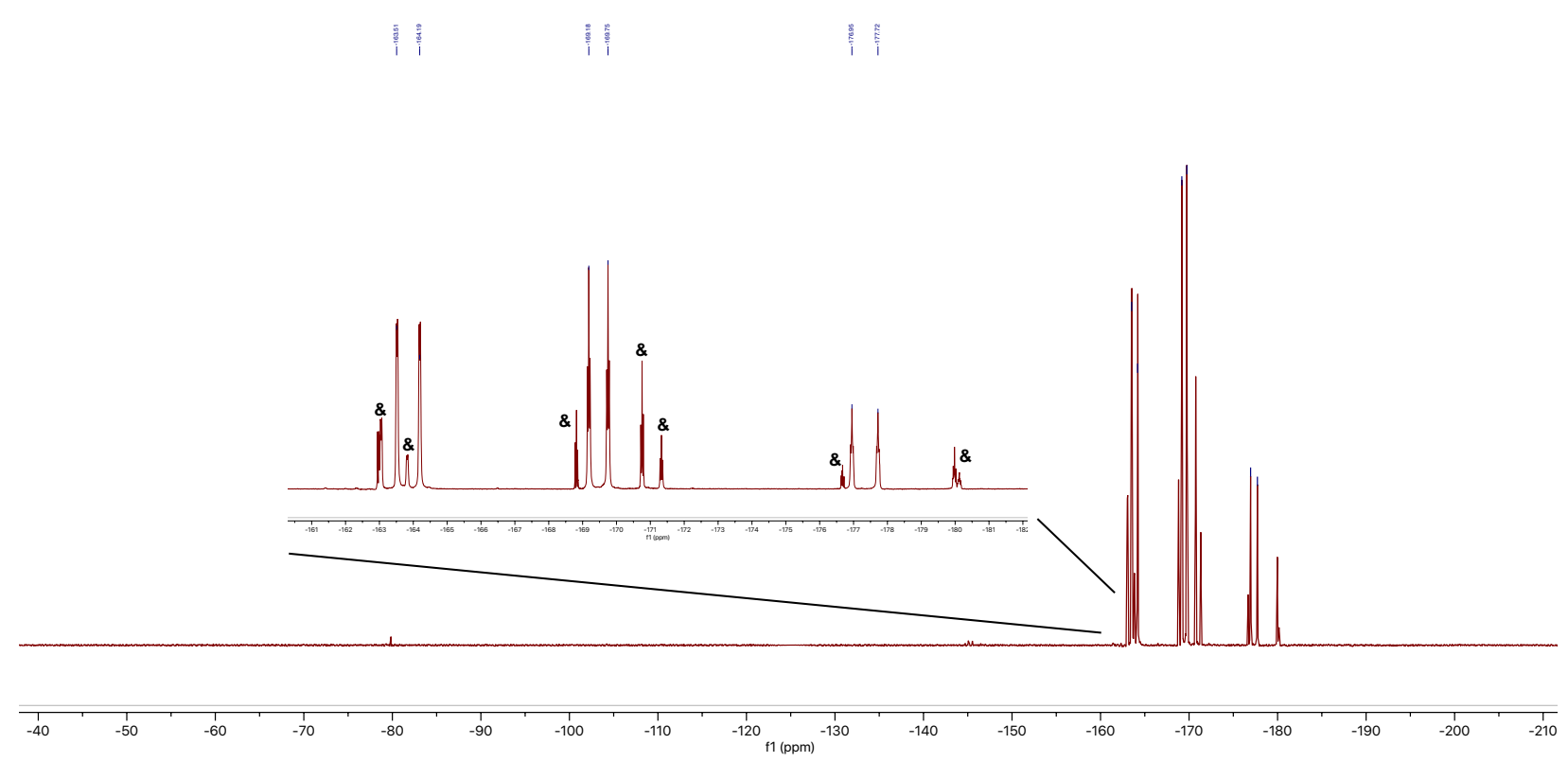

Figure S11. ${ }^{19} \mathrm{~F}\left\{{ }^{1} \mathrm{H}\right\}$ NMR spectrum of $\mathrm{Mo}(\mathrm{NAr})(\mathrm{CMePh})\left(\mathrm{OC}_{6} \mathrm{~F}_{5}\right)_{2}(\mathrm{MeCN})$ recorded at $564 \mathrm{MHz}$ in $\mathrm{CD}_{3} \mathrm{CN}$. The ampersand (\&) denotes resonances for isomers 1 and 2, which are proposed to be $\mathrm{Mo}(\mathrm{NAr})(\mathrm{CMePh})\left(\mathrm{OC}_{6} \mathrm{~F}_{5}\right)_{2}(\mathrm{MeCN})_{2}$ complexes. 


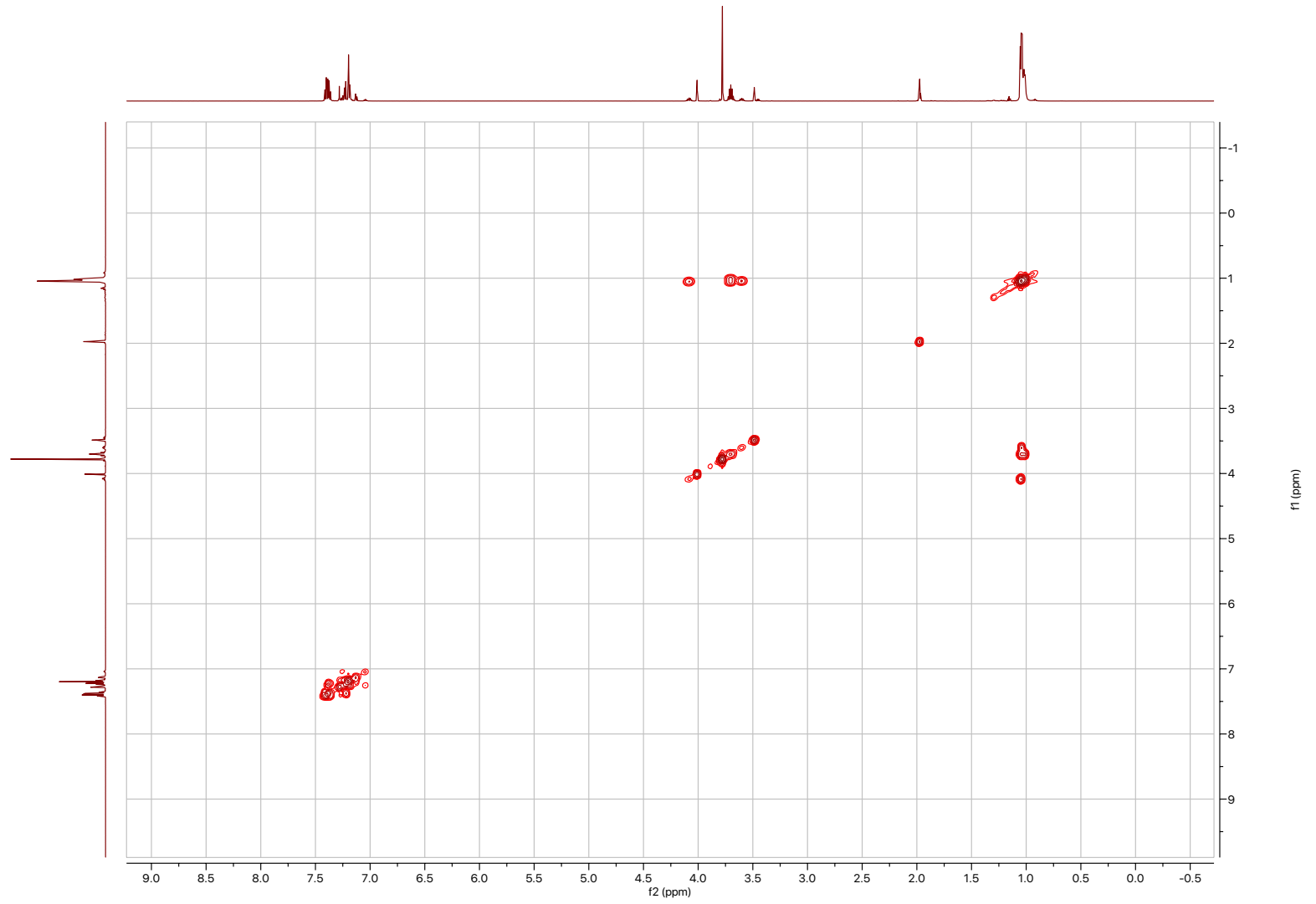

Figure S12. ${ }^{1} \mathrm{H},{ }^{1} \mathrm{H}-\mathrm{COSY} N M R$ spectrum of $\mathrm{Mo}(\mathrm{NAr})(\mathrm{CMePh})\left(\mathrm{OC}_{6} \mathrm{~F}_{5}\right)_{2}(\mathrm{MeCN})$ recorded at $600 \mathrm{MHz}$ in $\mathrm{CD}_{3} \mathrm{CN}$. 


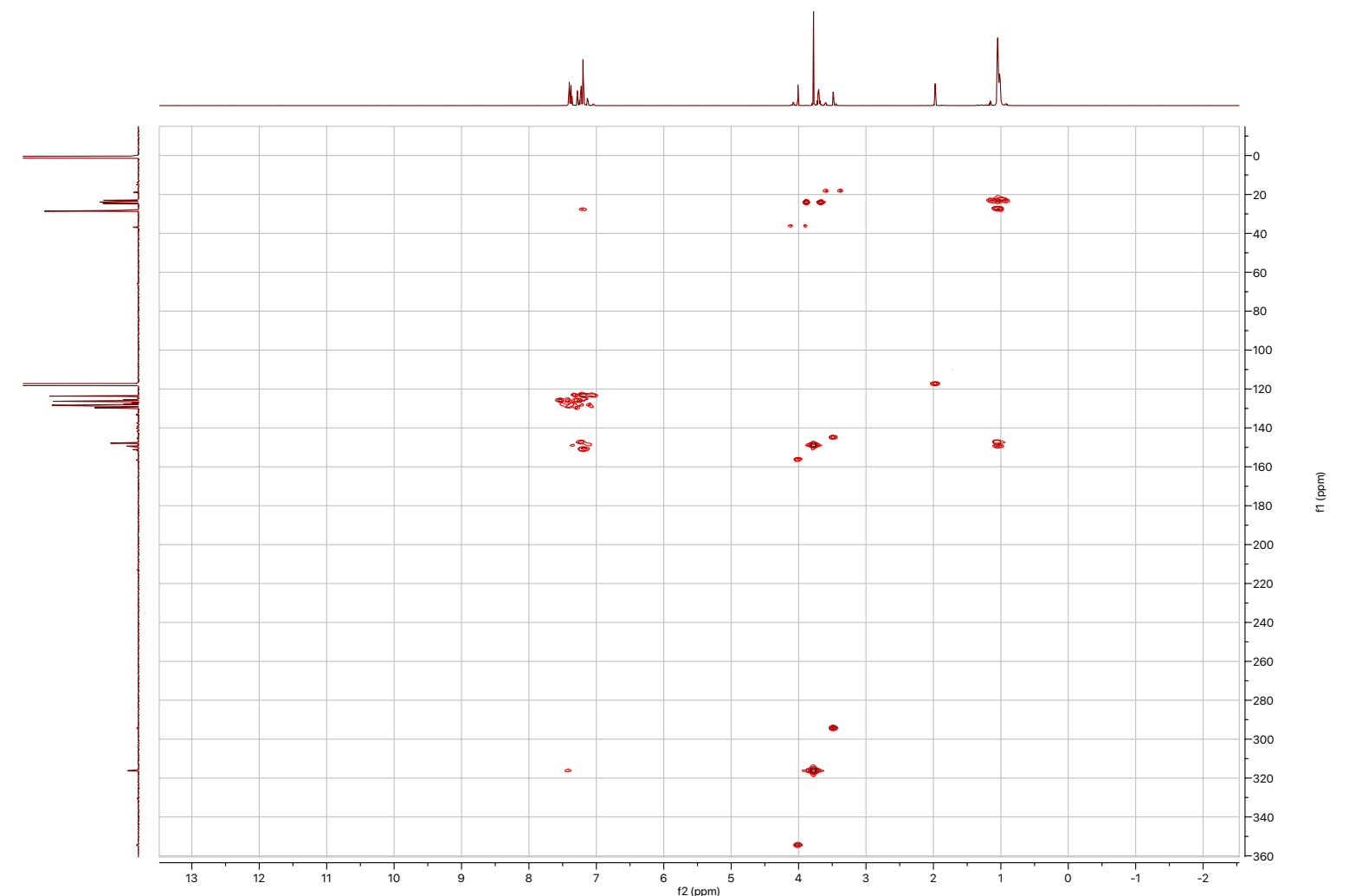

Figure S13. ${ }^{13} \mathrm{C},{ }^{1} \mathrm{H}-\mathrm{HMBC}$ NMR spectrum of $\mathrm{Mo}(\mathrm{NAr})(\mathrm{CMePh})\left(\mathrm{OC}_{6} \mathrm{~F}_{5}\right)_{2}(\mathrm{MeCN})$ recorded at $151 \mathrm{MHz}$ in $\mathrm{CD}_{3} \mathrm{CN}$.

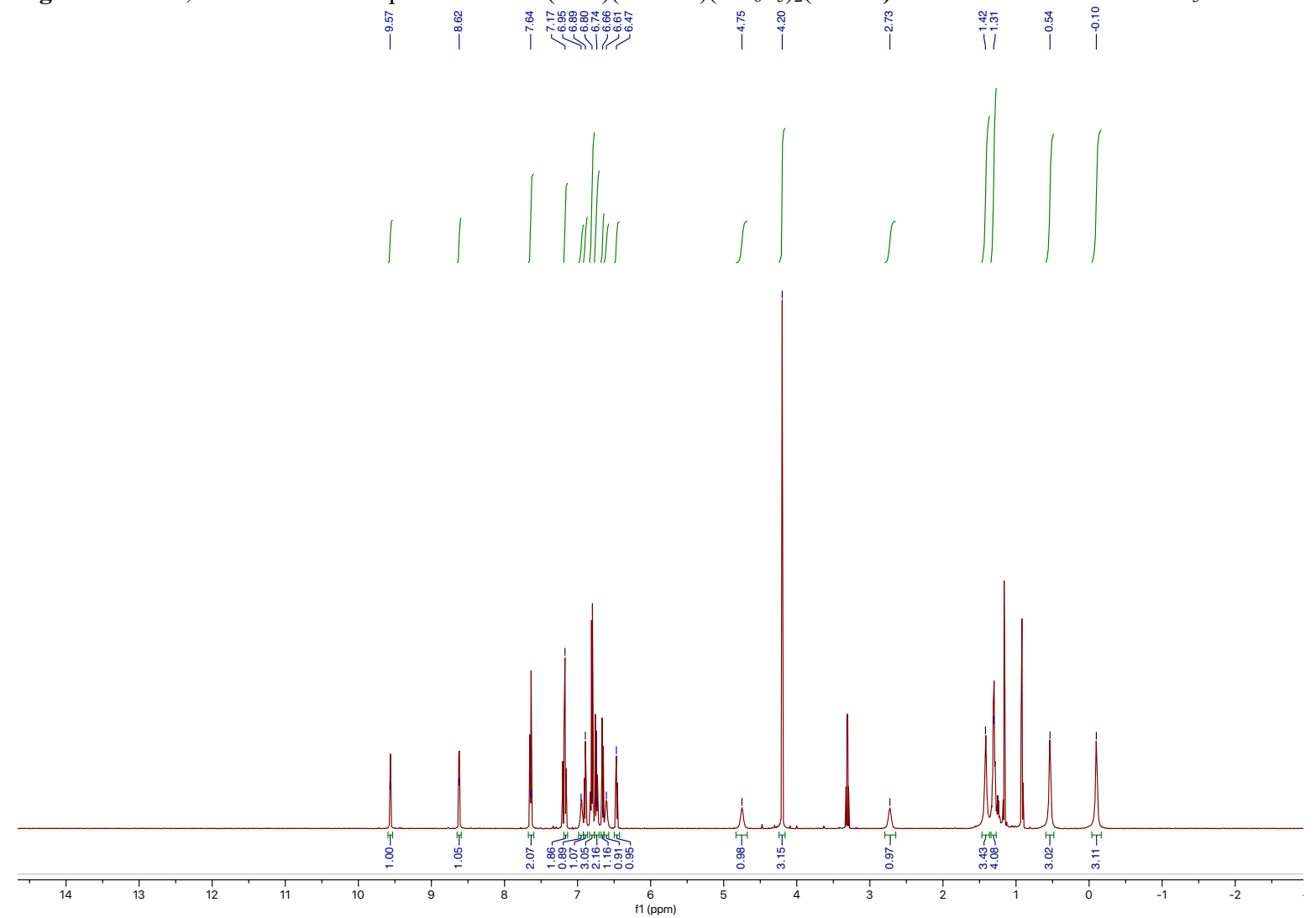

Figure S14. ${ }^{1} \mathrm{H}$ NMR spectrum of $\mathrm{Mo}(\mathrm{NAr})(\mathrm{CMePh})\left(\mathrm{OC}_{6} \mathrm{~F}_{5}\right)_{2}$ (bipy) recorded at $600 \mathrm{MHz}$ in $\mathrm{C}_{6} \mathrm{D}_{6}$. 


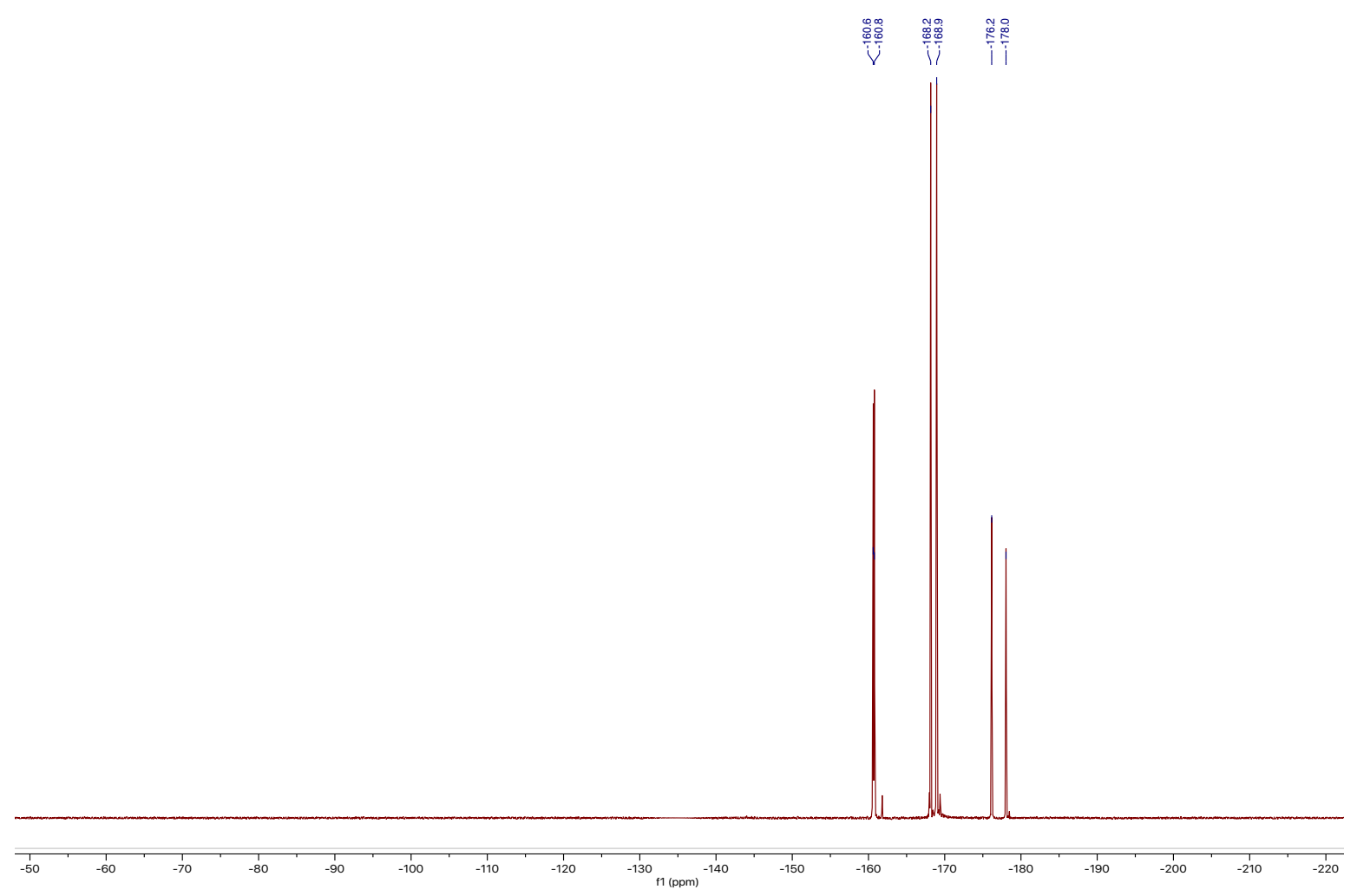

Figure S15. ${ }^{19} \mathrm{~F}\left\{{ }^{1} \mathrm{H}\right\}$ NMR spectrum of $\mathrm{Mo}(\mathrm{NAr})(\mathrm{CMePh})\left(\mathrm{OC}_{6} \mathrm{~F}_{5}\right)_{2}$ (bipy) recorded at $565 \mathrm{MHz}$ in $\mathrm{C}_{6} \mathrm{D}_{6}$.

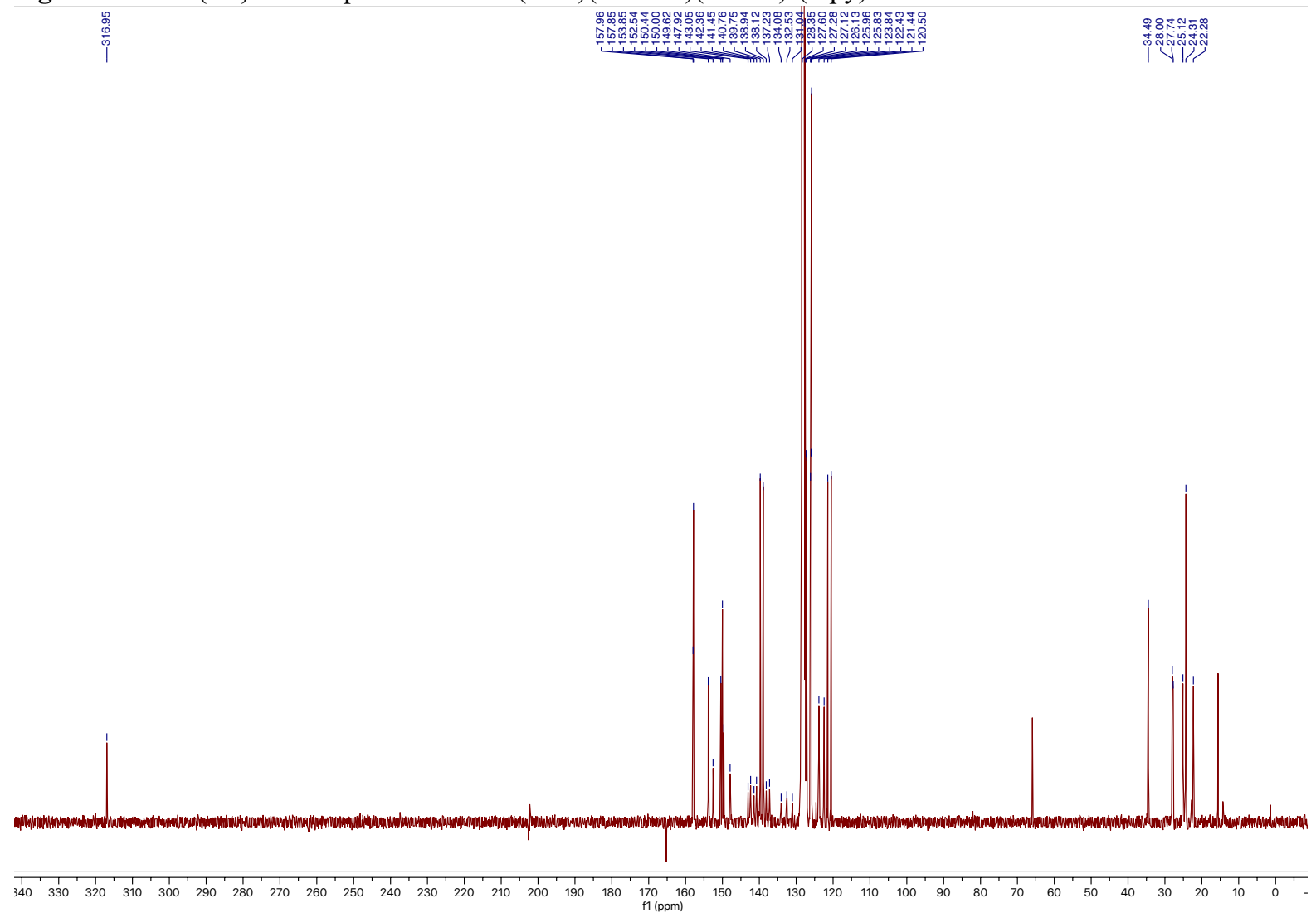

Figure S16. ${ }^{13} \mathrm{C}\left\{{ }^{1} \mathrm{H}\right\}$ NMR spectrum of $\mathrm{Mo}(\mathrm{NAr})(\mathrm{CMePh})\left(\mathrm{OC}_{6} \mathrm{~F}_{5}\right)_{2}$ (bipy) recorded at $151 \mathrm{MHz}$ in $\mathrm{C}_{6} \mathrm{D}_{6}$. 


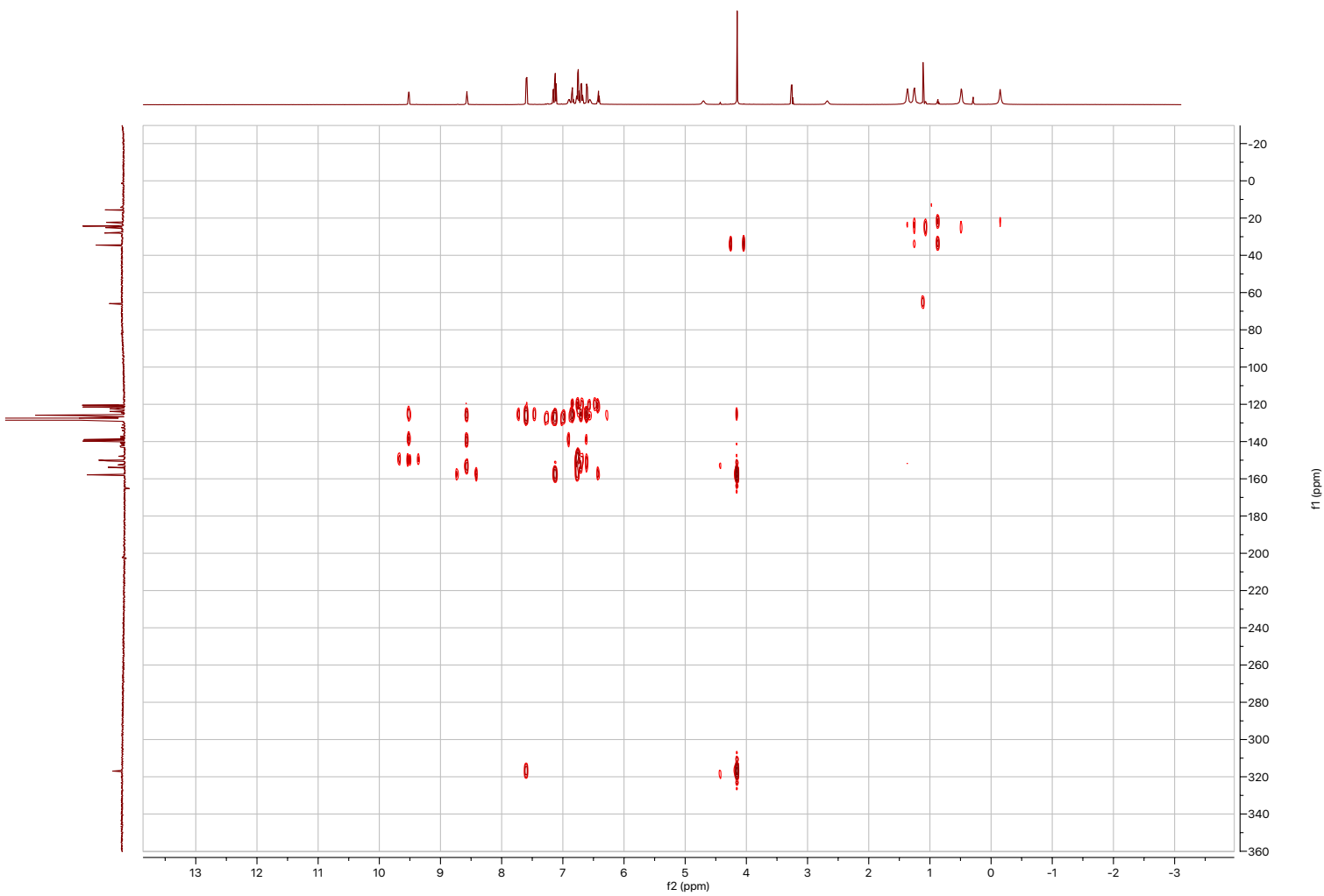

Figure S17. ${ }^{13} \mathrm{C},{ }^{1} \mathrm{H}-\mathrm{HMBC}$ NMR spectrum of $\mathrm{Mo}(\mathrm{NAr})(\mathrm{CMePh})\left(\mathrm{OC}_{6} \mathrm{~F}_{5}\right)_{2}$ (bipy) recorded at $151 \mathrm{MHz}$ in $\mathrm{C}_{6} \mathrm{D}_{6}$.

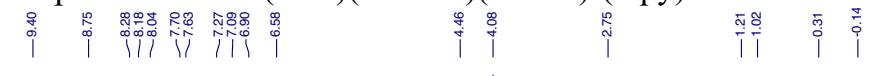

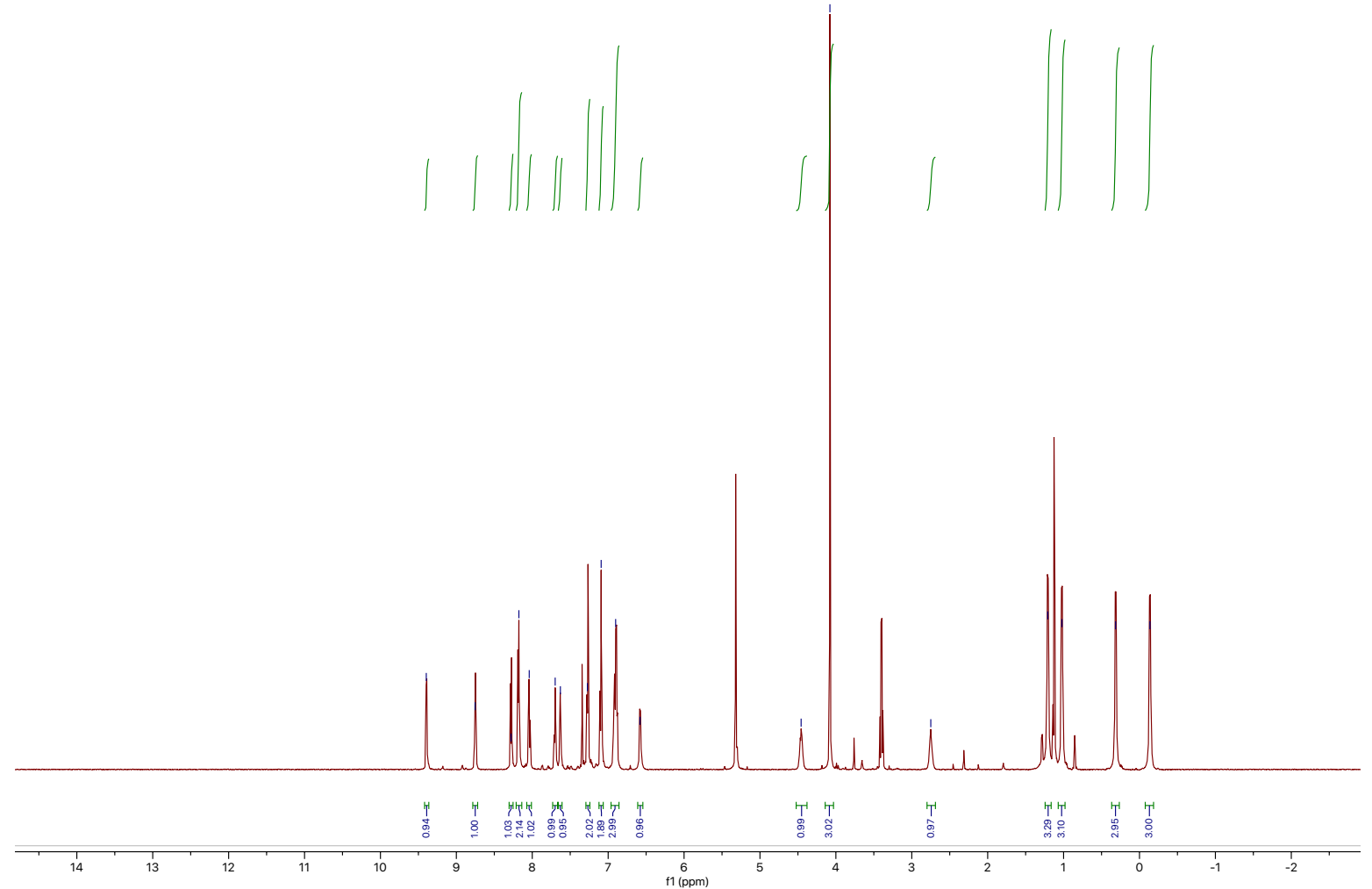

Figure S18. ${ }^{1} \mathrm{H}$ NMR spectrum of $\mathrm{Mo}(\mathrm{NAr})(\mathrm{CMePh})(\mathrm{Cl})_{2}\left(\right.$ bipy) recorded at $600 \mathrm{MHz}$ in $\mathrm{CD}_{2} \mathrm{Cl}_{2}$ at $-40{ }^{\circ} \mathrm{C}$. 


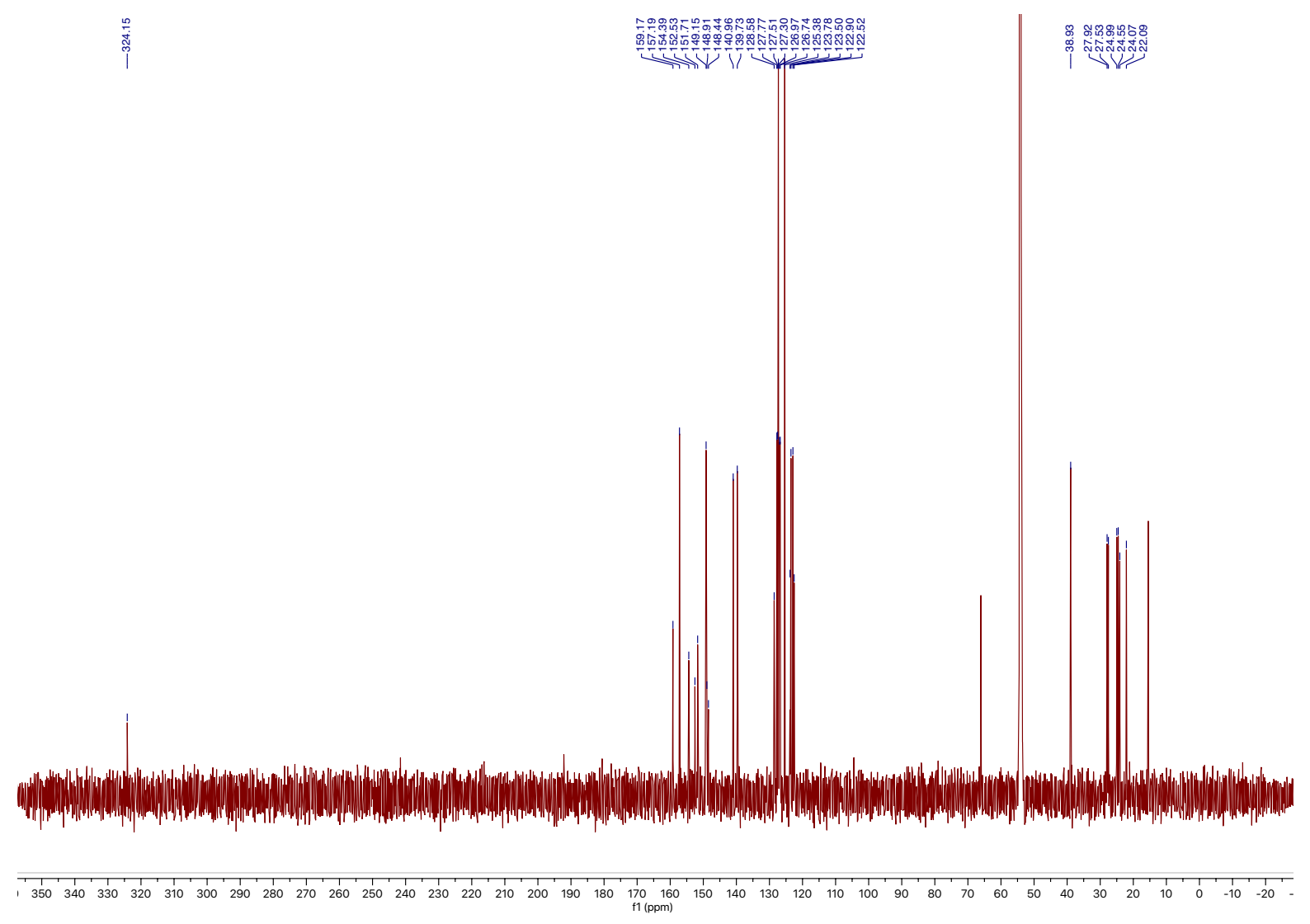

Figure S19. ${ }^{13} \mathrm{C}\left\{{ }^{1} \mathrm{H}\right\}$ NMR spectrum of $\mathrm{Mo}(\mathrm{NAr})(\mathrm{CMePh})(\mathrm{Cl})_{2}$ (bipy) recorded at $151 \mathrm{MHz}$ in $\mathrm{CD}_{2} \mathrm{Cl}_{2}$ at $-40{ }^{\circ} \mathrm{C}$.

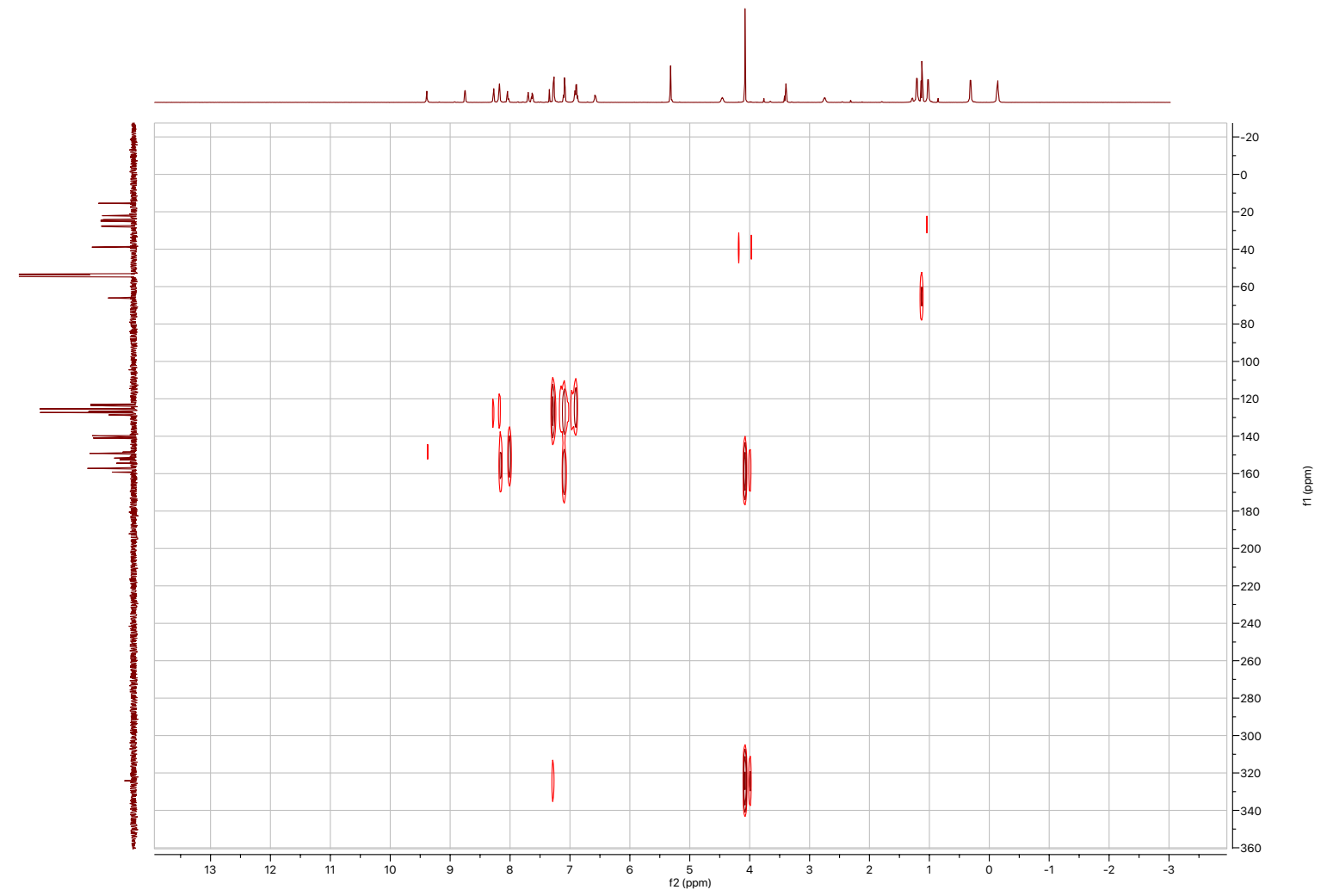

Figure S20. ${ }^{13} \mathrm{C},{ }^{1} \mathrm{H}-\mathrm{HMBC}$ NMR spectrum of $\mathrm{Mo}(\mathrm{NAr})(\mathrm{CMePh})(\mathrm{Cl})_{2}($ bipy $)$ recorded at $151 \mathrm{MHz}$ in $\mathrm{CD}_{2} \mathrm{Cl}_{2}$ at $-40{ }^{\circ} \mathrm{C}$. 


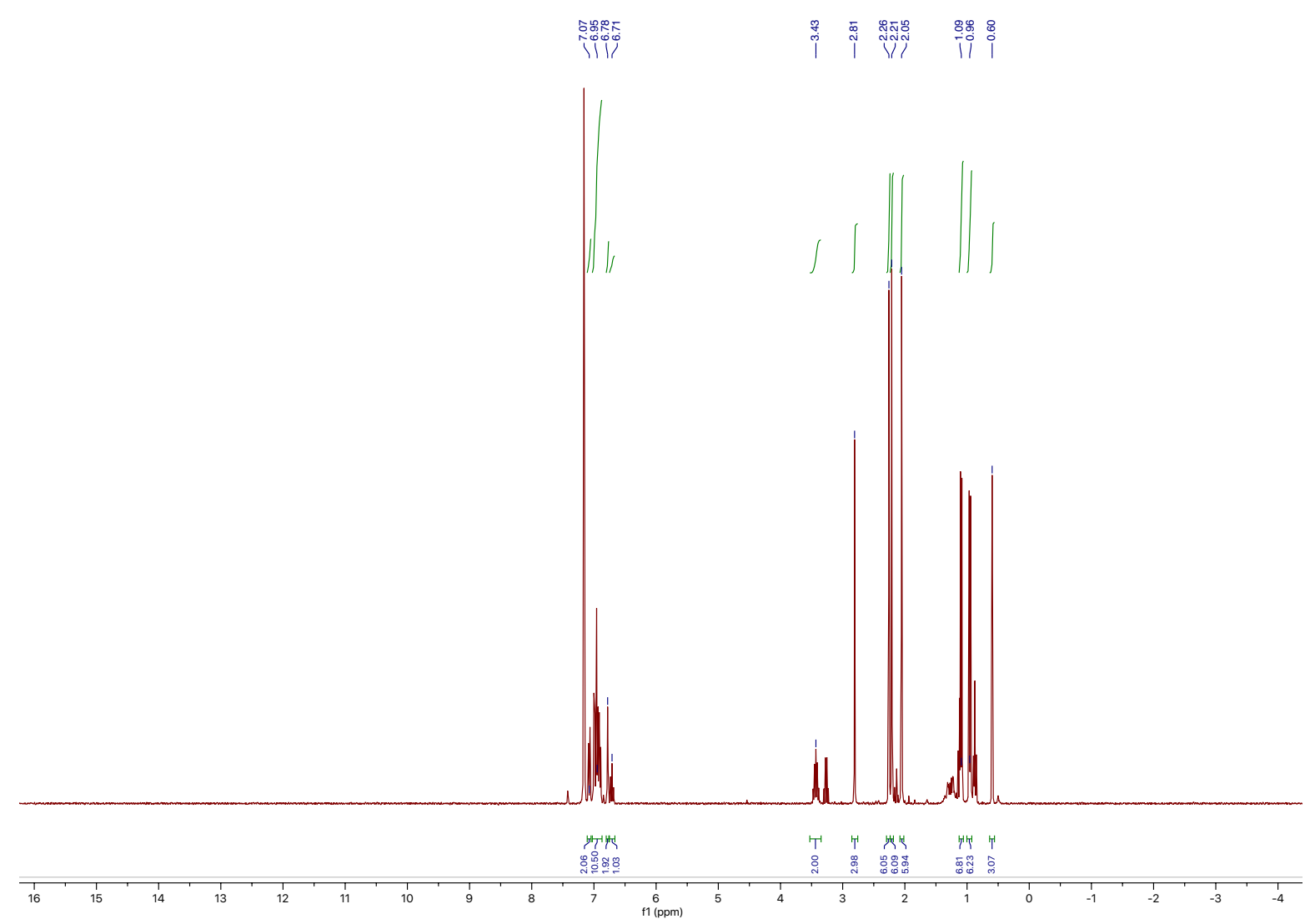

Figure S21. ${ }^{1} \mathrm{H}$ NMR spectrum of $\mathrm{Mo}(\mathrm{NAr})(\mathrm{CMePh})(\mathrm{Cl})(\mathrm{OHMT})(\mathrm{MeCN})$ recorded at $600 \mathrm{MHz}$ in $\mathrm{C}_{6} \mathrm{D}_{6}$.

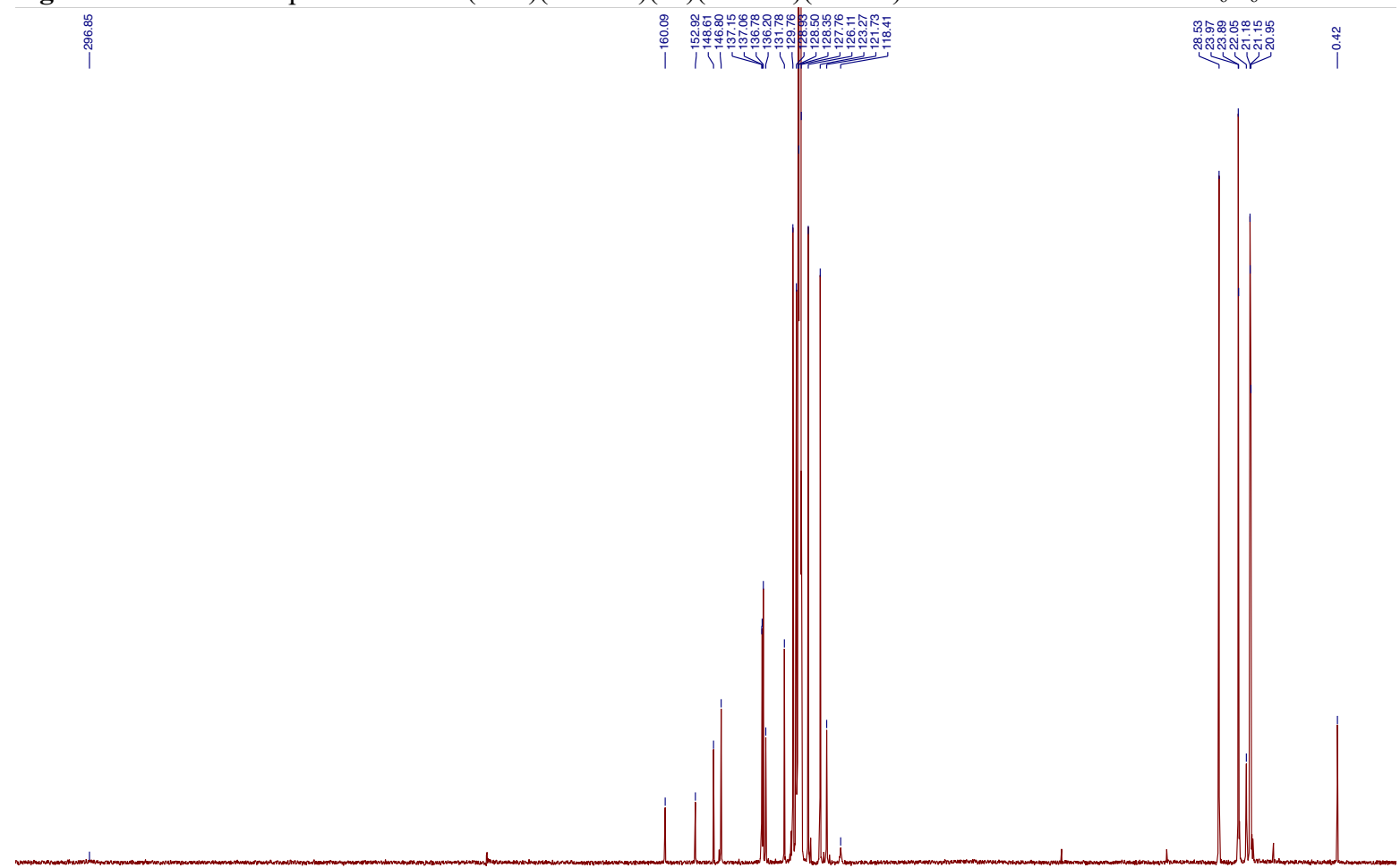

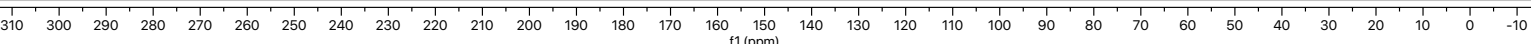

Figure S22. ${ }^{13} \mathrm{C}\left\{{ }^{1} \mathrm{H}\right\}$ NMR spectrum of $\mathrm{Mo}(\mathrm{NAr})(\mathrm{CMePh})(\mathrm{Cl})(\mathrm{OHMT})(\mathrm{MeCN})$ recorded at $151 \mathrm{MHz}$ in $\mathrm{C}_{6} \mathrm{D}_{6}$. 


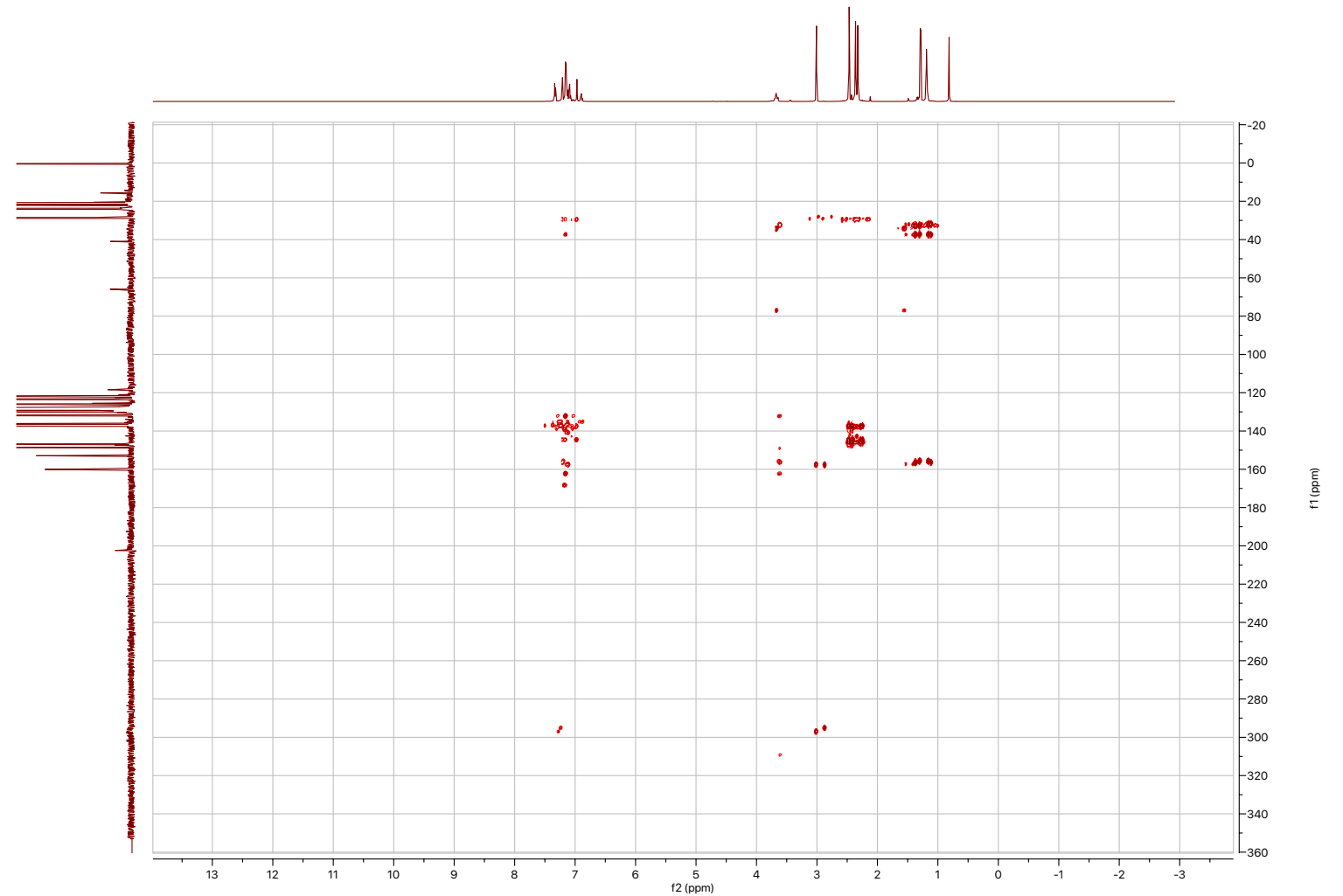

Figure S23. ${ }^{13} \mathrm{C},{ }^{1} \mathrm{H}-\mathrm{HMBC}$ NMR spectrum of $\mathrm{Mo}(\mathrm{NAr})(\mathrm{CMePh})(\mathrm{Cl})(\mathrm{OHMT})(\mathrm{MeCN})$ recorded at $151 \mathrm{MHz}$ in $\mathrm{C}_{6} \mathrm{D}_{6}$.

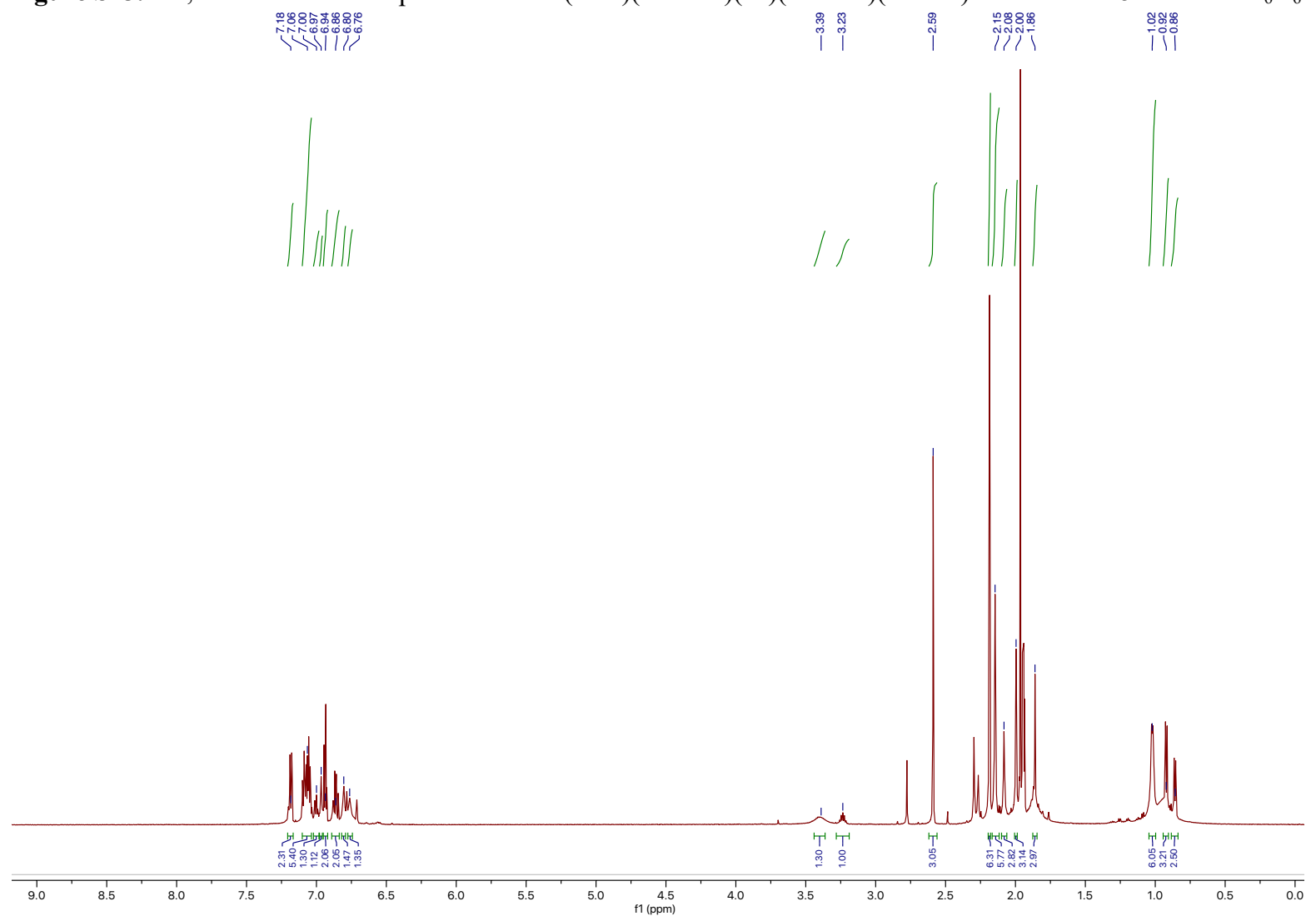

Figure S24. ${ }^{1} \mathrm{H}$ NMR spectrum of $\mathrm{Mo}(\mathrm{NAr})(\mathrm{CMePh})(\mathrm{Cl})(\mathrm{OHMT})(\mathrm{MeCN})$ recorded at $600 \mathrm{MHz}$ in $\mathrm{CD}_{3} \mathrm{CN}$. 


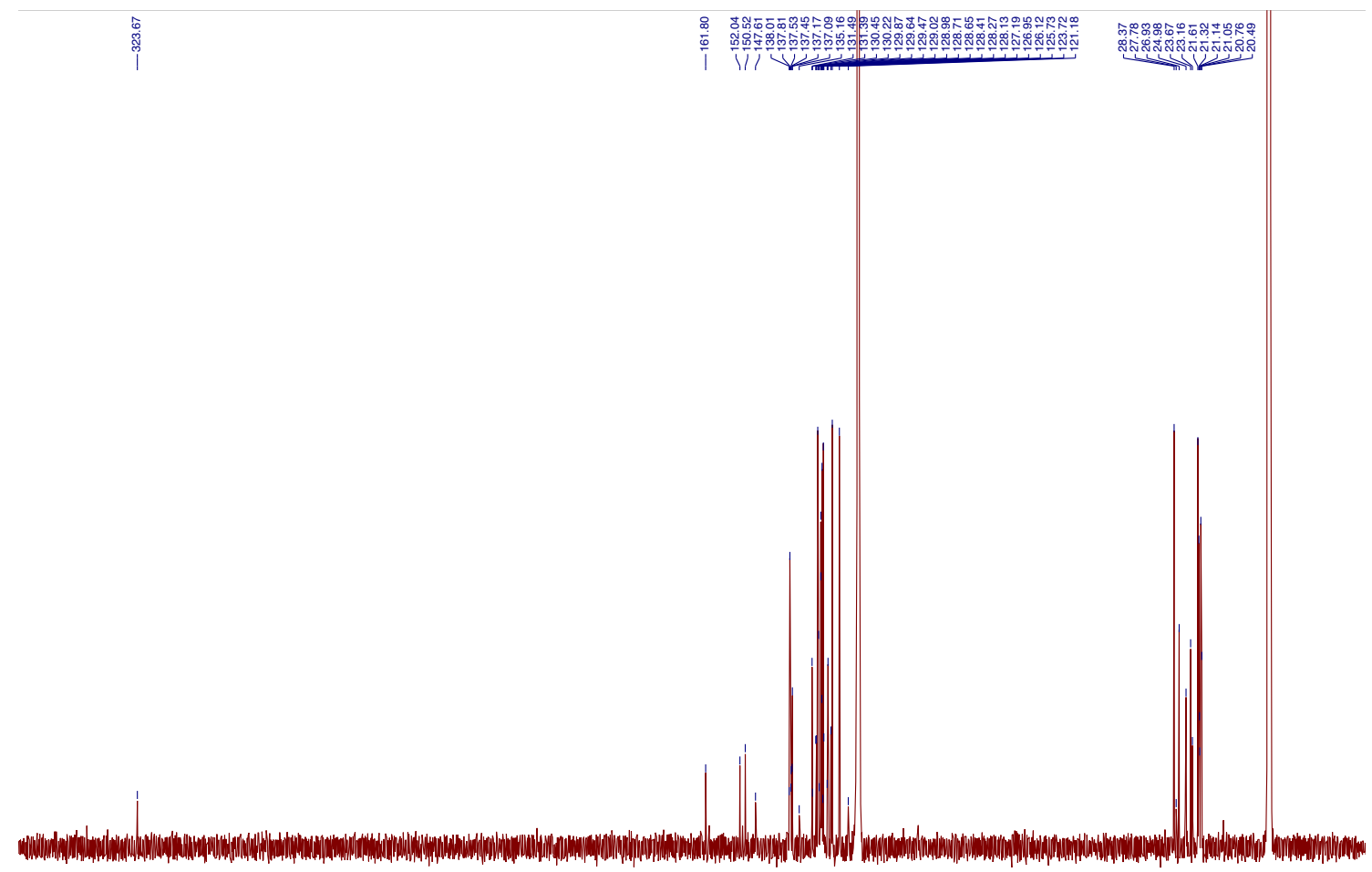

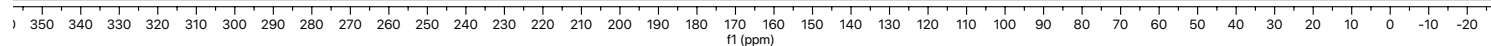

Figure S25. ${ }^{13} \mathrm{C}\left\{{ }^{1} \mathrm{H}\right\}$ NMR spectrum of $\mathrm{Mo}(\mathrm{NAr})(\mathrm{CMePh})(\mathrm{Cl})(\mathrm{OHMT})(\mathrm{MeCN})$ recorded at $151 \mathrm{MHz}$ in $\mathrm{CD}{ }_{3} \mathrm{CN}$.

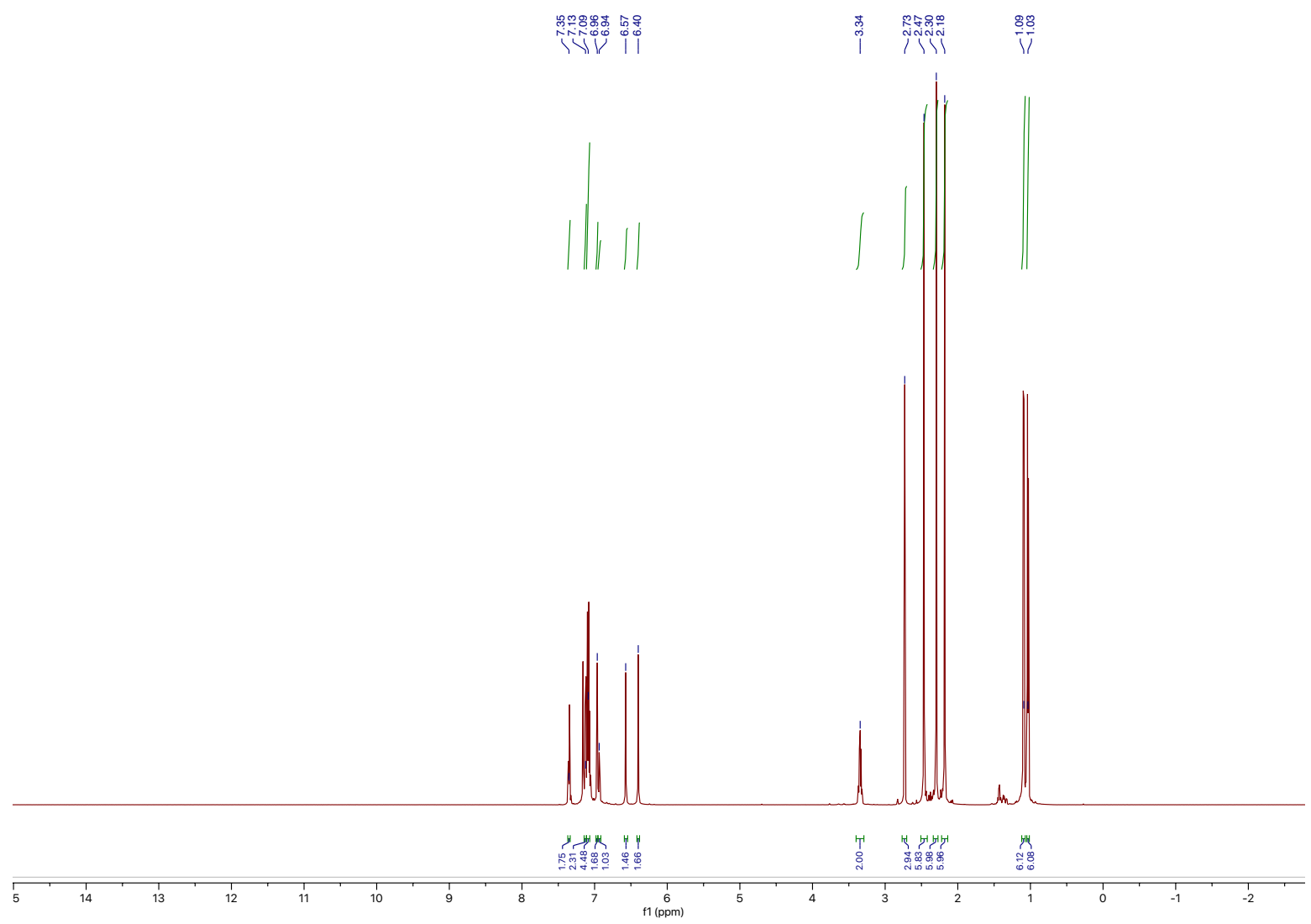

Figure S26. ${ }^{1} \mathrm{H}$ NMR spectrum of $\mathrm{Mo}(\mathrm{NAr})(\mathrm{CMePh})(\mathrm{OHMT})\left(\mathrm{NC}_{4} \mathrm{H}_{4}\right)$ recorded at $600 \mathrm{MHz}$ in $\mathrm{C}_{6} \mathrm{D}_{6}$. 


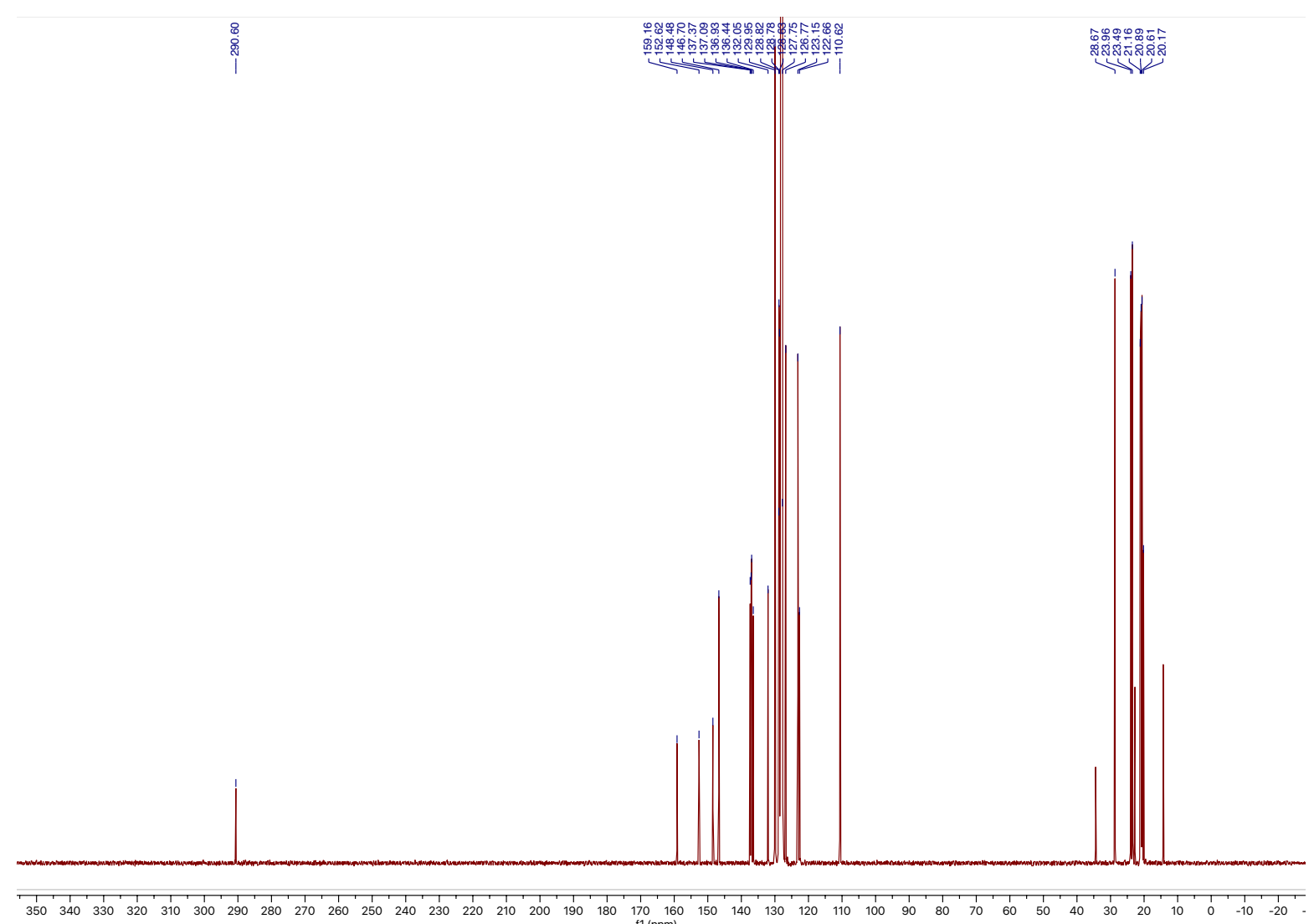

Figure S27. ${ }^{13} \mathrm{C}\left\{{ }^{1} \mathrm{H}\right\}$ NMR spectrum of $\mathrm{Mo}(\mathrm{NAr})(\mathrm{CMePh})(\mathrm{OHMT})\left(\mathrm{NC}_{4} \mathrm{H}_{4}\right)$ recorded at $151 \mathrm{MHz}$ in $\mathrm{C}_{6} \mathrm{D}_{6}$.

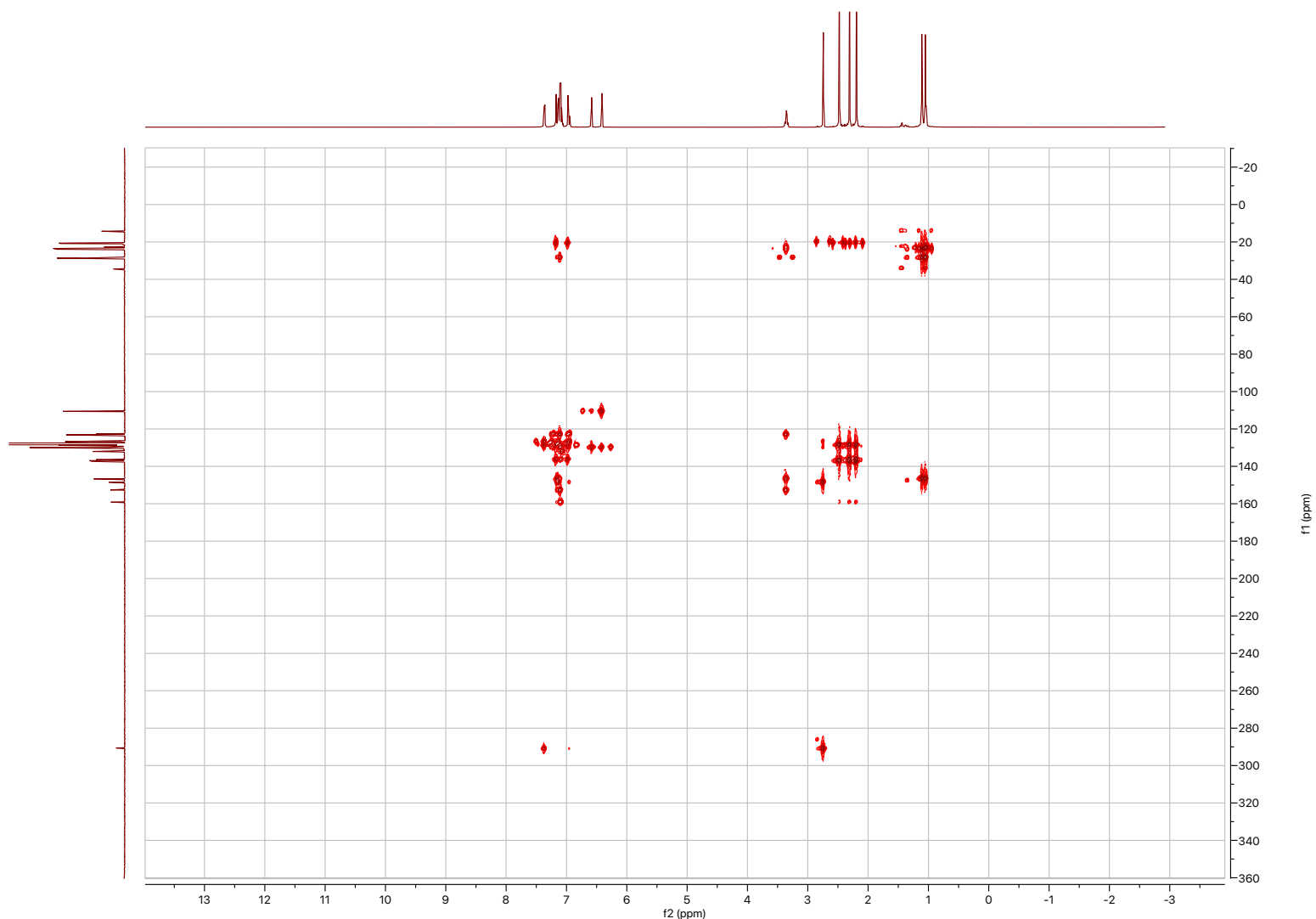

Figure S28. ${ }^{13} \mathrm{C},{ }^{1} \mathrm{H}-\mathrm{HMBC}$ NMR spectrum of $\mathrm{Mo}(\mathrm{NAr})(\mathrm{CMePh})(\mathrm{OHMT})\left(\mathrm{NC}_{4} \mathrm{H}_{4}\right)$ recorded at $151 \mathrm{MHz}$ in $\mathrm{C}_{6} \mathrm{D}_{6}$. S20 


\section{Preliminary studies of reactions of disubstituted alkylidene complexes with olefins.}

\section{Reaction of $2(\mathrm{THF})_{\mathrm{x}}$ with ethylene.}

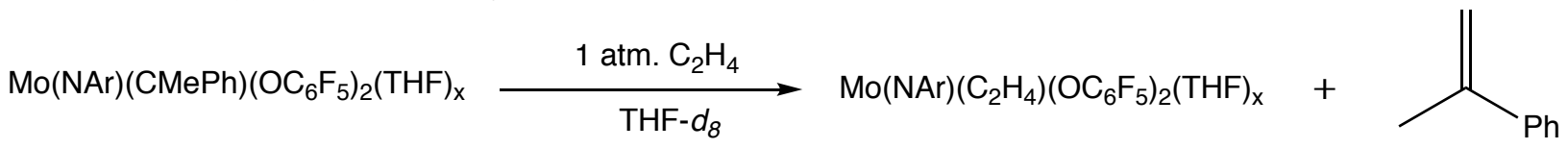

$\left[\mathrm{Mo}(\mathrm{NAr})(\mathrm{CMePh})\left(\mathrm{OC}_{6} \mathrm{~F}_{5}\right)_{2}\right]_{2}(0.007 \mathrm{~g}, 0.0094 \mathrm{mmol})$ was dissolved in THF- $d_{8}(0.6 \mathrm{~mL})$ to give an orange solution of $\mathrm{Mo}(\mathrm{NAr})(\mathrm{CMePh})\left(\mathrm{OC}_{6} \mathrm{~F}_{5}\right)_{2}(\mathrm{THF})_{\mathrm{x}}$ that was freeze-pump-thawed three times prior to addition of 1 atm of ethylene. After 2 hours, the volatiles were removed from the dark green solution in vacuo $\left(10^{-2}\right.$ mbar, 10 minutes $)$ prior to dissolving the residue in THF- $d_{8}$ and collecting NMR spectra. Crystallization of what is proposed to be $\mathrm{Mo}(\mathrm{NAr})\left(\mathrm{C}_{2} \mathrm{H}_{4}\right)\left(\mathrm{OC}_{6} \mathrm{~F}_{5}\right)_{2}(\mathrm{THF})_{\mathrm{x}}$ was hindered by its gradual decomposition in the absence of ethylene to unidentified products. ${ }^{1} \mathrm{H}$ NMR $\left(600 \mathrm{MHz}, \mathrm{THF}-d_{8}\right) \delta 7.1(\mathrm{dd}, J=8.9,6.2 \mathrm{~Hz}, 1 \mathrm{H}), 7.1-7.1(\mathrm{~m}, 2 \mathrm{H}), 3.6(\mathrm{~s}, 4 \mathrm{H}), 2.6-2.6$ $(\mathrm{m}, 2 \mathrm{H}), 2.2-2.1(\mathrm{~m}, 2 \mathrm{H}), 1.7(\mathrm{~s}, 4 \mathrm{H}), 1.1(\mathrm{~d}, J=6.8 \mathrm{~Hz}, 12 \mathrm{H}) .{ }^{19} \mathrm{~F}\left\{{ }^{1} \mathrm{H}\right\}$ NMR $(282 \mathrm{MHz}$, THF$\left.d_{8}\right) \delta-159.9--160.4(\mathrm{~m}),-168.3(\mathrm{t}, J=21.1 \mathrm{~Hz}),-177.5(\mathrm{ddd}, J=29.5,21.3,7.2 \mathrm{~Hz}) .{ }^{13} \mathrm{C}\left\{{ }^{1} \mathrm{H}\right\}$ NMR $\left(151 \mathrm{MHz}, \mathrm{THF}-d_{8}\right) \delta 153.5,149.5,145.4,143.3,141.7,140.7-139.6(\mathrm{~m}), 138.6(\mathrm{t}, J=$ $13.8 \mathrm{~Hz}), 134.0(\mathrm{t}, J=12.6 \mathrm{~Hz}), 132.4(\mathrm{t}, J=16.2 \mathrm{~Hz}), 129.8,129.1,128.6,126.2,124.1,68.4$, $64.0,28.7,26.5,24.9$.

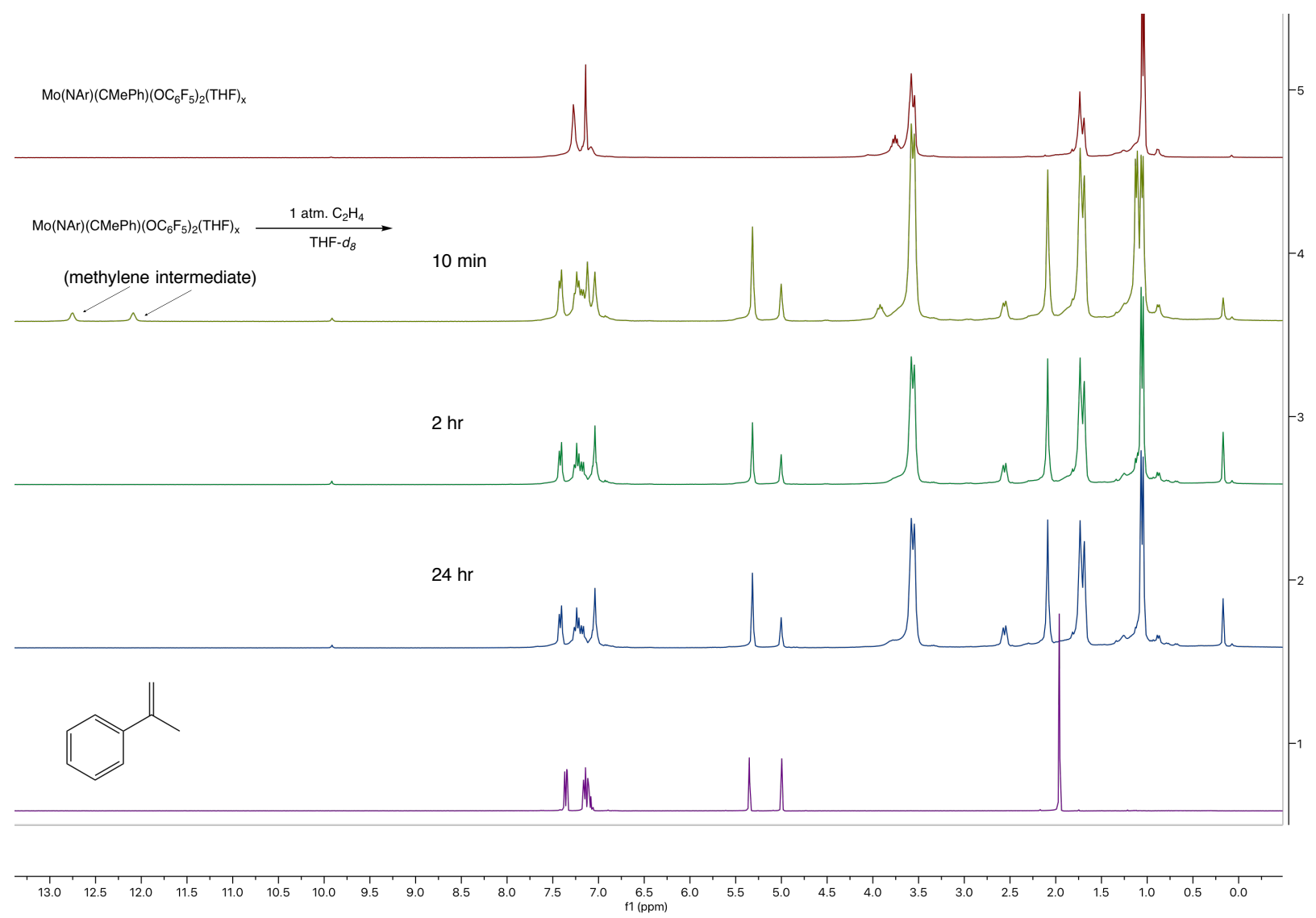

Figure S29. ${ }^{1} \mathrm{H}$ NMR spectra monitoring the progress of the reaction of $\mathrm{Mo}(\mathrm{NAr})(\mathrm{CMePh})\left(\mathrm{OC}_{6} \mathrm{~F}_{5}\right)_{2}(\mathrm{THF})_{\mathrm{x}}$ with 1 atm ethylene. Spectrum 5 is pure $\mathrm{Mo}(\mathrm{NAr})(\mathrm{CMePh})\left(\mathrm{OC}_{6} \mathrm{~F}_{5}\right)_{2}(\mathrm{THF})_{\mathrm{x}}$, spectra 2-4 track the reaction over 24 hours with time of collection inlayed. Spectra 1 is pure $\alpha$-methylstyrene. All spectra were recorded at $300 \mathrm{MHz}$ in THF- $d 8$ at $22{ }^{\circ} \mathrm{C}$. 


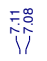

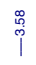

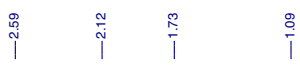

$\mu$

$1 \int 1$

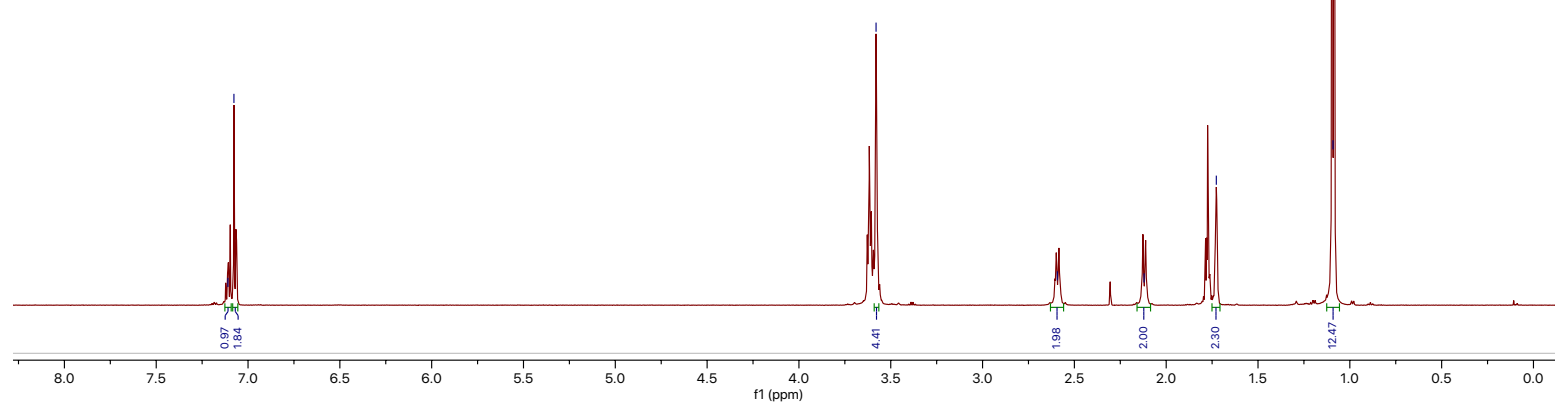

Figure S30. ${ }^{1} \mathrm{H}$ NMR spectrum of $\mathrm{Mo}(\mathrm{NAr})\left(\mathrm{C}_{2} \mathrm{H}_{4}\right)\left(\mathrm{OC}_{6} \mathrm{~F}_{5}\right)_{2}(\mathrm{THF})_{\mathrm{x}}$ recorded at $600 \mathrm{MHz}$ in THF- $d 8$.

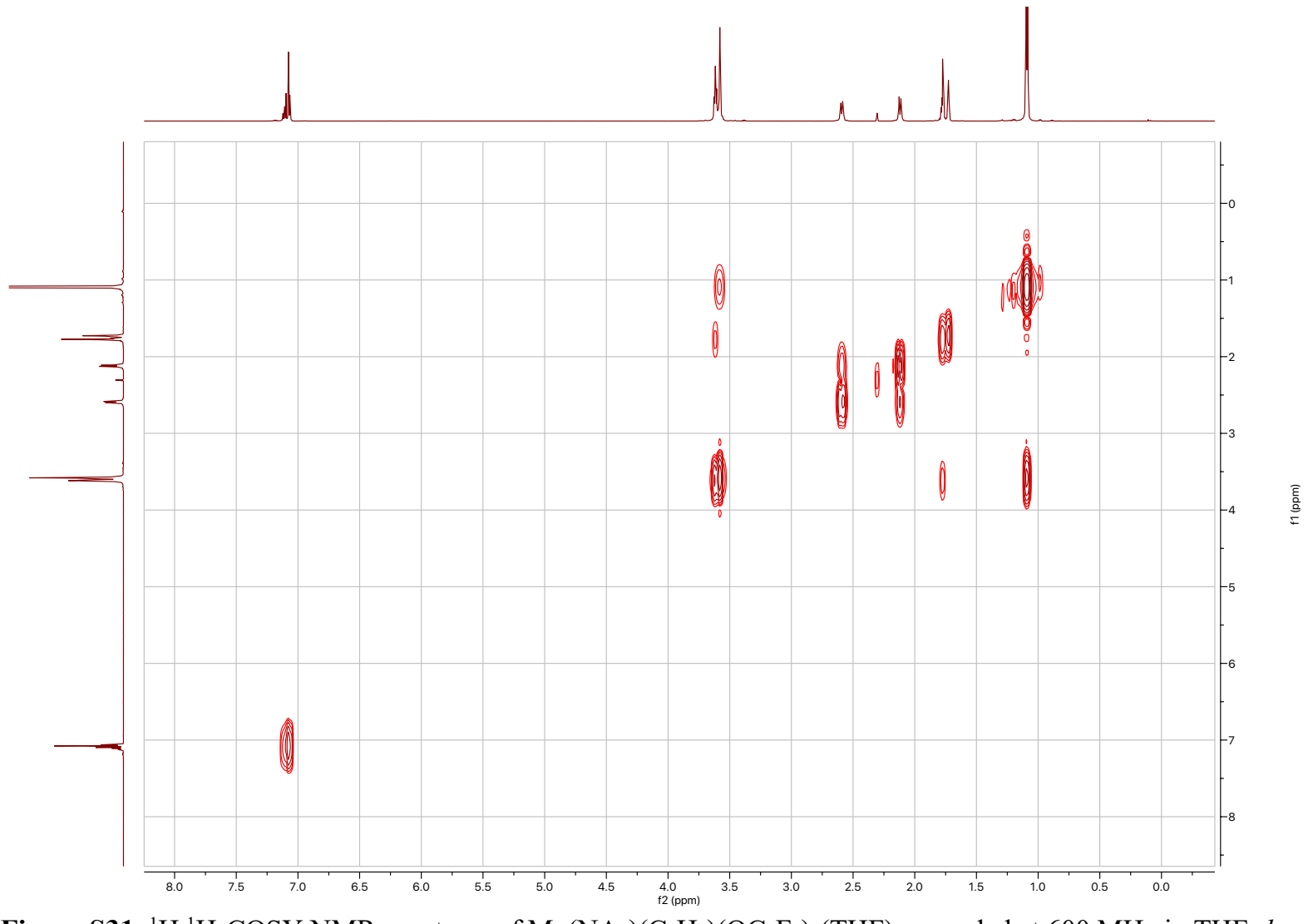

Figure S31. ${ }^{1} \mathrm{H},{ }^{1} \mathrm{H}-\mathrm{COSY}$ NMR spectrum of $\mathrm{Mo}(\mathrm{NAr})\left(\mathrm{C}_{2} \mathrm{H}_{4}\right)\left(\mathrm{OC}_{6} \mathrm{~F}_{5}\right)_{2}(\mathrm{THF})_{\mathrm{x}}$ recorded at $600 \mathrm{MHz}$ in THF-d8.

$\mathrm{S} 22$ 


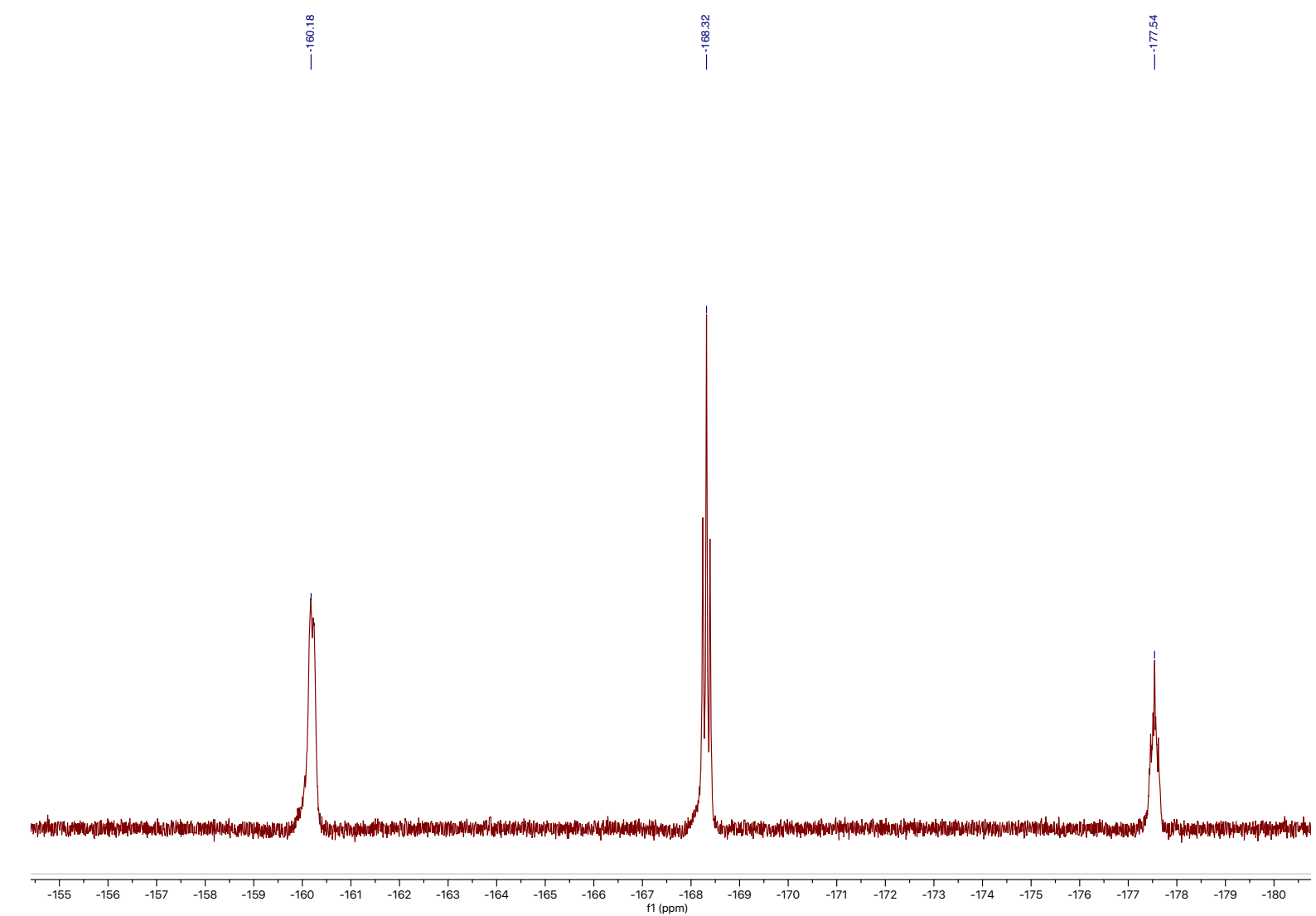

Figure S32. ${ }^{19} \mathrm{~F}\left\{{ }^{1} \mathrm{H}\right\}$ NMR spectrum of $\mathrm{Mo}(\mathrm{NAr})\left(\mathrm{C}_{2} \mathrm{H}_{4}\right)\left(\mathrm{OC}_{6} \mathrm{~F}_{5}\right)_{2}(\mathrm{THF})_{\mathrm{x}}$ recorded at $282 \mathrm{MHz}$ in THF- $d 8$.

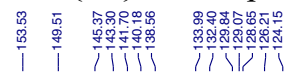

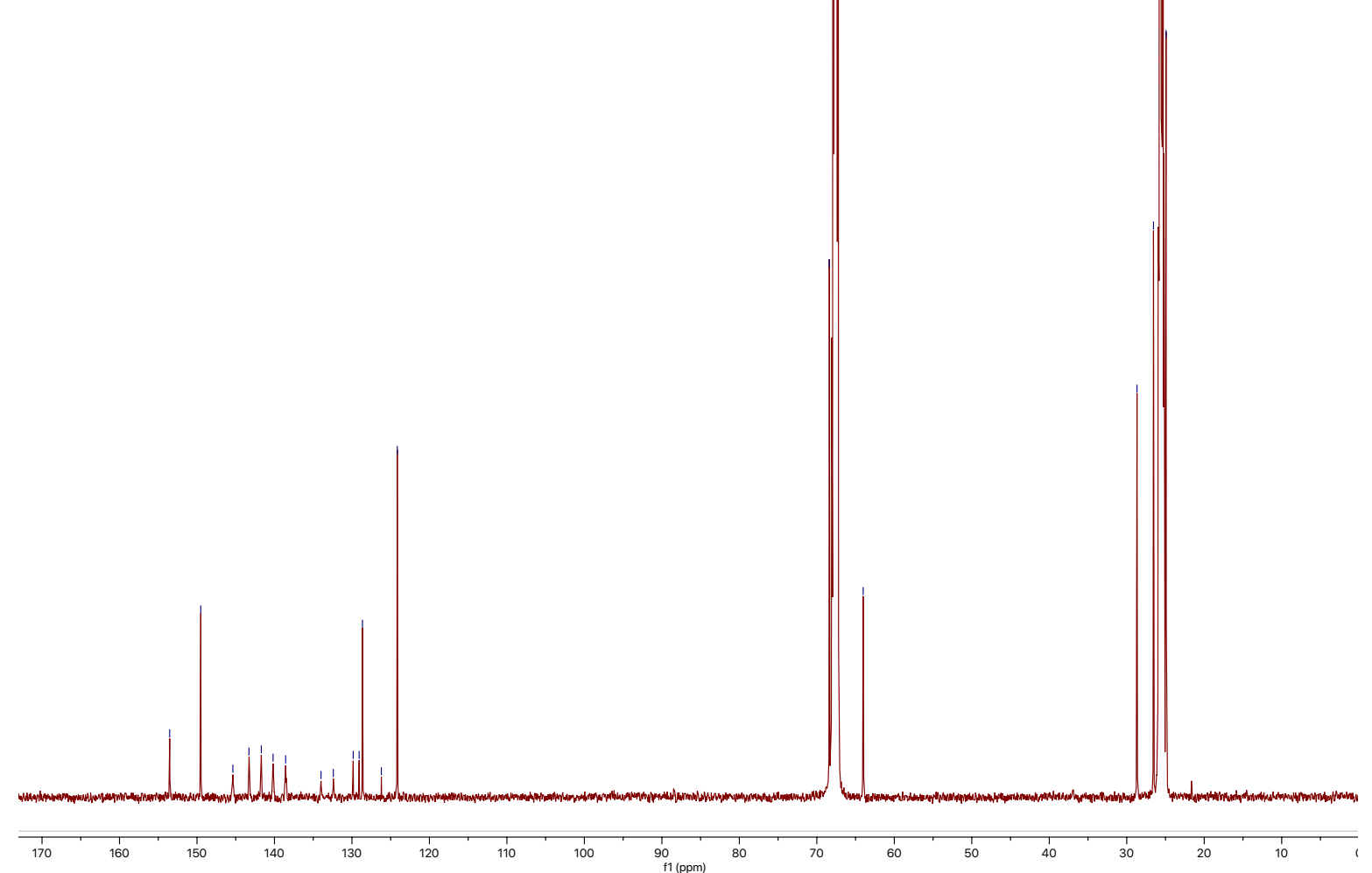

Figure S33. ${ }^{13} \mathrm{C}\left\{{ }^{1} \mathrm{H}\right\}$ NMR spectrum of $\mathrm{Mo}(\mathrm{NAr})\left(\mathrm{C}_{2} \mathrm{H}_{4}\right)\left(\mathrm{OC}_{6} \mathrm{~F}_{5}\right)_{2}(\mathrm{THF})_{\mathrm{x}}$ recorded at $151 \mathrm{MHz}$ in THF- $d 8$. 


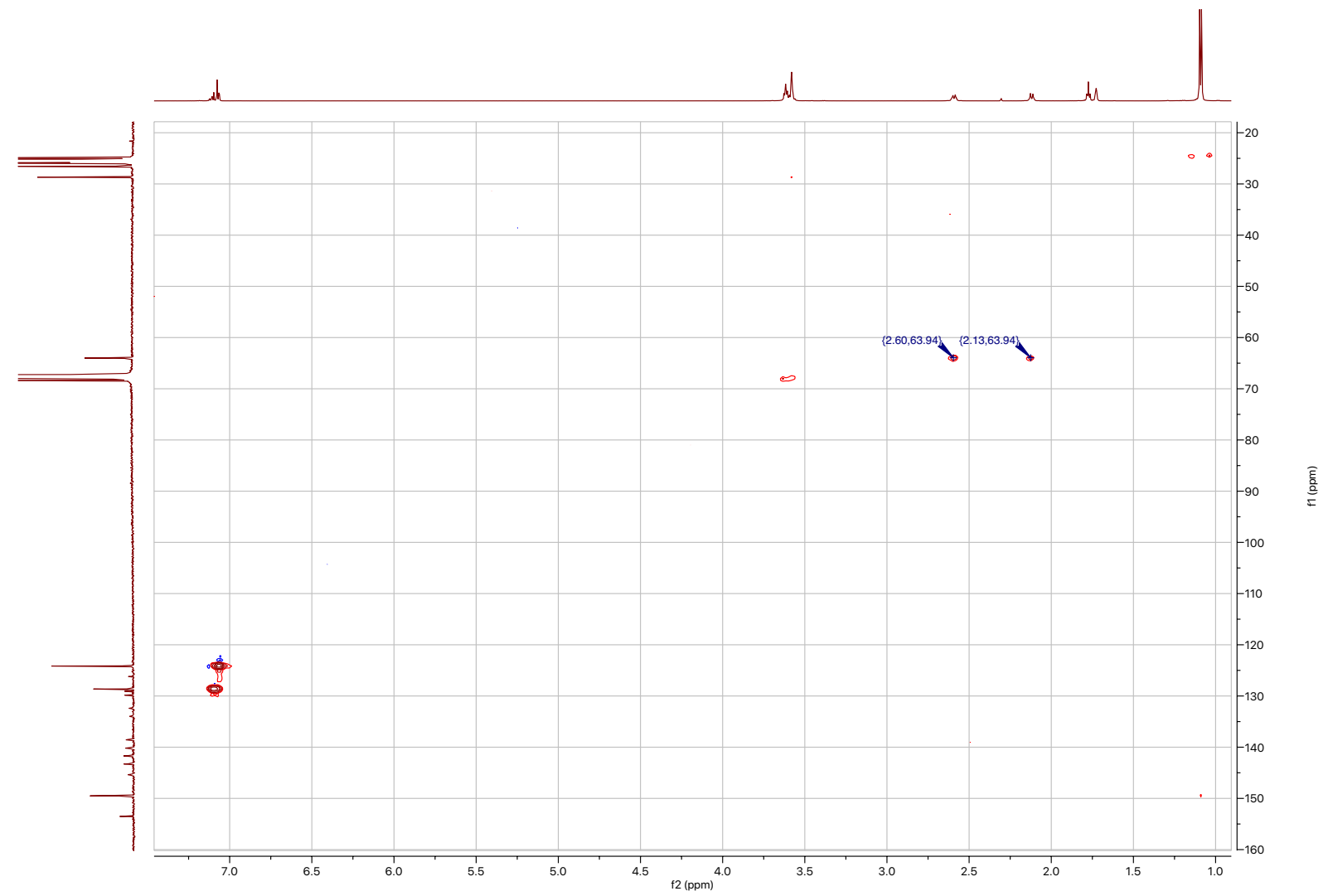

Figure S34. ${ }^{13} \mathrm{C},{ }^{1} \mathrm{H}-\mathrm{HSQC}$ NMR spectrum of $\mathrm{Mo}(\mathrm{NAr})\left(\mathrm{C}_{2} \mathrm{H}_{4}\right)\left(\mathrm{OC}_{6} \mathrm{~F}_{5}\right)_{2}(\mathrm{THF})_{\mathrm{x}}$ recorded at $151 \mathrm{MHz}$ in THF-d8. 


\section{Reaction of $4(\mathrm{MeCN})$ with ethylene}<smiles>C=C(C)c1ccccc1</smiles>

$\mathrm{Mo}(\mathrm{NAr})(\mathrm{CMePh})(\mathrm{Cl})(\mathrm{OHMT})(\mathrm{MeCN})(0.007 \mathrm{~g}, 0.0094 \mathrm{mmol})$ was dissolved in $\mathrm{C}_{6} \mathrm{D}_{6}(0.6$ $\mathrm{mL}$ ) and the solution was freeze-pump-thawed three times before addition of 1 atm of ethylene. $\alpha$-Methylstyrene was observed by ${ }^{1} \mathrm{H}$ NMR after 10 minutes.

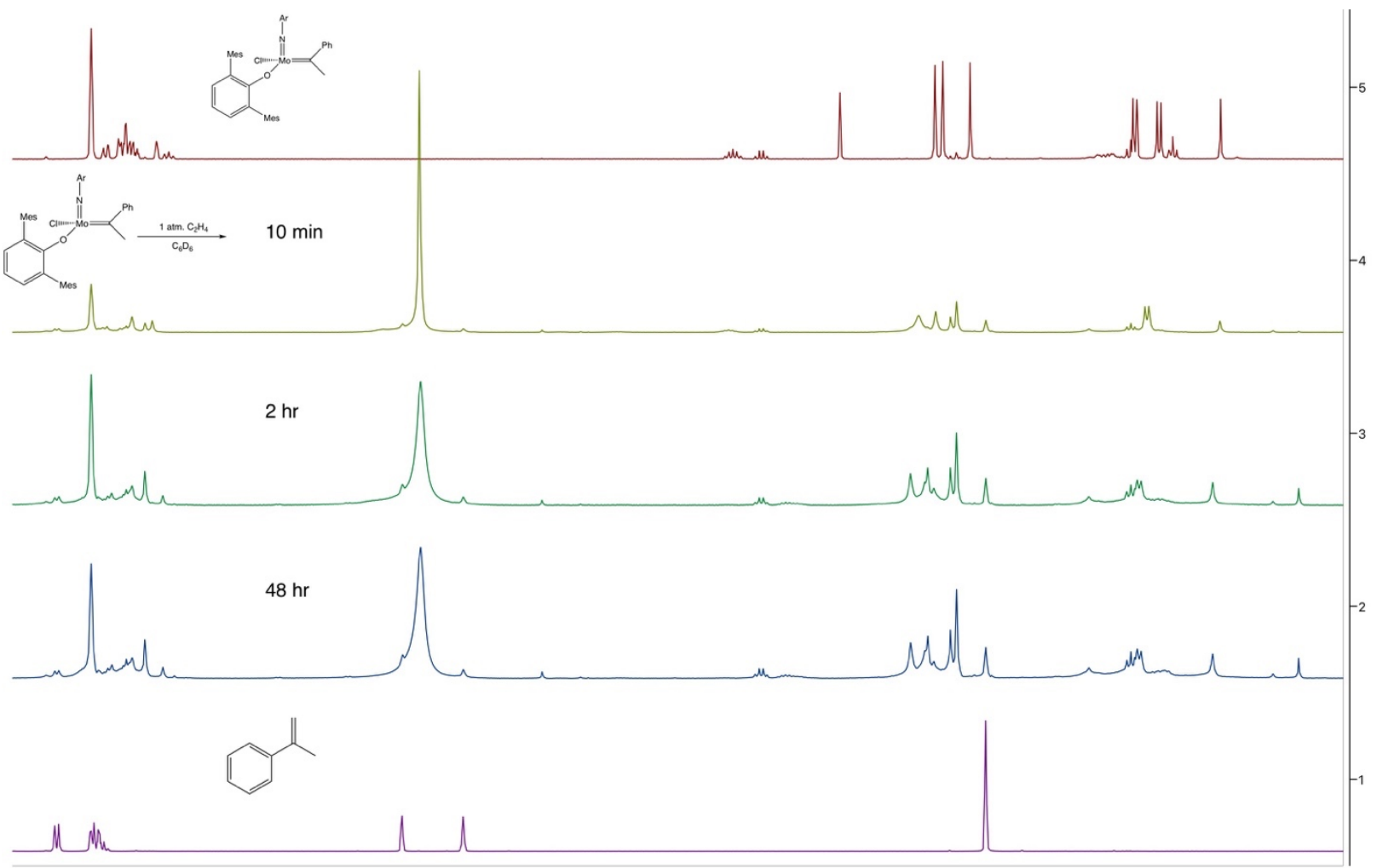

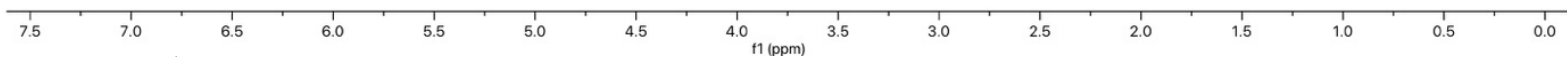

Figure S35. ${ }^{1} \mathrm{H}$ NMR spectra monitoring the progress of the reaction of $\mathrm{Mo}(\mathrm{NAr})(\mathrm{CMePh})(\mathrm{Cl})(\mathrm{OHMT})(\mathrm{MeCN})$ with $1 \mathrm{~atm}$ of ethylene. Spectrum 5 is pure $\mathrm{Mo}(\mathrm{NAr})(\mathrm{CMePh})(\mathrm{Cl})(\mathrm{OHMT})(\mathrm{MeCN})$, spectra 2-4 track the reaction progress over 48 hours. Spectrum 1 is pure $\alpha$-methylstyrene. All spectra were recorded at $300 \mathrm{MHz}$ in $\mathrm{C}_{6} \mathrm{D}_{6}$ at 22 ${ }^{\circ} \mathrm{C}$. 


\section{Ring-opening polymerization of cyclooctene}

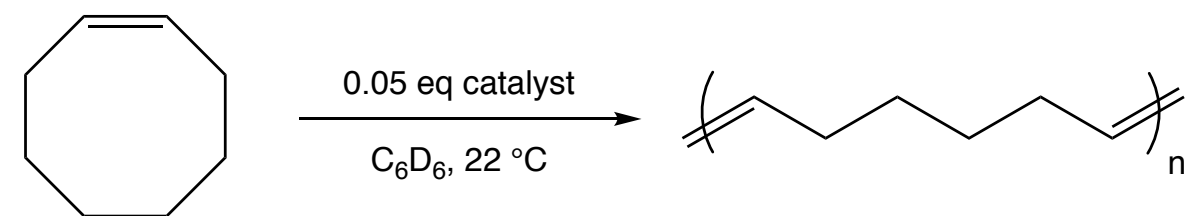

Polymerization with $\mathrm{Mo}(\mathrm{NAr})(\mathrm{CMePh})(\mathrm{Cl})(\mathrm{OHMT})(\mathrm{MeCN})$ as the initiator. $\mathrm{A} \mathrm{C}_{6} \mathrm{D}_{6}(0.1 \mathrm{~mL})$ solution of $\mathrm{Mo}(\mathrm{NAr})(\mathrm{CMePh})(\mathrm{Cl})(\mathrm{OHMT})(\mathrm{MeCN})(0.001 \mathrm{~g}, 0.0014 \mathrm{mmol})$ in $\mathrm{C}_{6} \mathrm{D}_{6}(0.1 \mathrm{~mL})$ was added cyclooctene $(2.52 \mu \mathrm{L}, 0.022 \mathrm{mmol})$ via microsyringe. The reaction was stirred in an open vial at $22{ }^{\circ} \mathrm{C}$. Aliquots were taken periodically that were diluted with $\mathrm{C}_{6} \mathrm{D}_{6}(0.5 \mathrm{~mL})$ prior to collecting ${ }^{1} \mathrm{H}$ NMR spectra. Conversion was estimated by integration of the olefin proton resonance of cyclooctene (m, 5.69-5.61 ppm) and polycyclooctene (m, 5.51-5.45 ppm).

Polymerization with $\mathrm{Mo}(\mathrm{NAr})(\mathrm{CMePh})\left(\mathrm{OC}_{6} \mathrm{~F}_{5}\right)_{2}(\mathrm{THF})_{\mathrm{x}}$ as the initiator. $\left[\mathrm{Mo}(\mathrm{NAr})(\mathrm{CMePh})\left(\mathrm{OC}_{6} \mathrm{~F}_{5}\right)_{2}\right]_{2}(0.001 \mathrm{~g}, 0.0013 \mathrm{mmol})$ was dissolved in THF- $d_{8}(100 \mu \mathrm{L})$ and diluted with $\mathrm{C}_{6} \mathrm{D}_{6}(900 \mu \mathrm{L})$ prior to adding cyclooctene $(2.39 \mu \mathrm{L}, 0.026 \mathrm{mmol})$ via microsyringe. The reaction was stirred in an open vial at $22^{\circ} \mathrm{C}$. Aliquots were taken periodically that were diluted with $\mathrm{C}_{6} \mathrm{D}_{6}(0.5 \mathrm{~mL})$ prior to collecting ${ }^{1} \mathrm{H}$ NMR spectra. Conversion was estimated by integration of the olefin proton resonance of cyclooctene $(\mathrm{m}, 5.69-5.61 \mathrm{ppm})$ and polycyclooctene $(\mathrm{m}, 5.51$ $5.45 \mathrm{ppm})$.

Table S1. Conversion of cyclooctene to polycyclooctene.

\begin{tabular}{|l|c|c|}
\hline \multicolumn{1}{|c|}{ Inititator/Conversion (\%) } & 20 minutes & 2 hours \\
\hline $\mathrm{Mo}(\mathrm{NAr})(\mathrm{CMePh})(\mathrm{Cl})(\mathrm{OHMT})(\mathrm{MeCN})$ & $>99$ & $>99$ \\
\hline $\mathrm{Mo}(\mathrm{NAr})(\mathrm{CMePh})\left(\mathrm{OC}_{6} \mathrm{~F}_{5}\right)_{2}(\mathrm{THF})_{\mathrm{x}}$ & 97 & $>99$ \\
\hline
\end{tabular}




\section{Homocoupling of 1-decene}

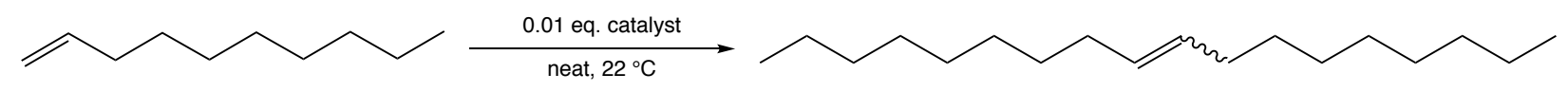

Homocoupling with $\mathrm{Mo}(\mathrm{NAr})(\mathrm{CMePh})(\mathrm{Cl})(\mathrm{OHMT})(\mathrm{MeCN})$ as the initiator. 1-Decene (118 $\mu \mathrm{L}, 0.60 \mathrm{mmol})$ was added via microsyringe to $\mathrm{Mo}(\mathrm{NAr})(\mathrm{CMePh})(\mathrm{Cl})(\mathrm{OHMT})(\mathrm{MeCN})(0.005 \mathrm{~g}$, $0.006 \mathrm{mmol}$ ) at $22{ }^{\circ} \mathrm{C}$. The reaction was stirred in an open vial. Aliquots were removed periodically and diluted with $0.5 \mathrm{~mL} \mathrm{CDCl}_{3}$ prior to collecting ${ }^{1} \mathrm{H}$ NMR spectra. Conversion was estimated by integration of the olefin proton resonance of 1-decene ( $\mathrm{m}, 5.86-5.78)$ and 9octadecene $(\mathrm{m}, 5.39-5.32)$. The $\mathrm{Z} / \mathrm{E}$ ratio was estimated by integration of the olefin proton resonance of E-9-octadecene ( $\mathrm{m}, 5.39-5.37)$ and Z-9-octadecene (m, 5.37-5.32).

Homocoupling with $\mathrm{Mo}(\mathrm{NAr})(\mathrm{CMePh})\left(\mathrm{OC}_{6} \mathrm{~F}_{5}\right)_{2}(\mathrm{THF})_{\mathrm{x}}$ as the initiator. $\left[\mathrm{Mo}(\mathrm{NAr})(\mathrm{CMePh})\left(\mathrm{OC}_{6} \mathrm{~F}_{5}\right)_{2}\right]_{2}(0.005 \mathrm{~g}, 0.007 \mathrm{mmol})$ was dissolved in THF- $d_{8}(100 \mu \mathrm{L})$ prior to adding 1-decene $(133 \mu \mathrm{L}, 0.70 \mathrm{mmol})$ via microsyringe. The reaction was stirred in an open vial. Aliquots were removed periodically and diluted with $0.5 \mathrm{~mL} \mathrm{CDCl}_{3}$ prior to collecting ${ }^{1} \mathrm{H} \mathrm{NMR}$ spectra. Conversion was estimated by integration of the olefin proton resonance of 1-decene $(\mathrm{m}$, 5.86-5.78) and 9-octadecene $(\mathrm{m}, 5.39-5.32)$. The $\mathrm{Z} / \mathrm{E}$ ratio was estimated by integration of the olefin proton resonance of E-9-octadecene ( $\mathrm{m}, 5.39-5.37)$ and Z-9-octadecene (m, 5.37-5.32).

Table S2. Conversion of 1-decene to 9-octadecene and Z/E ratio of the product.

\begin{tabular}{|c|c|c|c|c|}
\hline Inititator/Conversion, \% & 20 minutes & 2 hours & 24 hours & Final Z/E \\
\hline $\mathrm{Mo}(\mathrm{NAr})(\mathrm{CMePh})(\mathrm{Cl})(\mathrm{OHMT})(\mathrm{MeCN})$ & 86 & 91 & 91 & $37 / 63$ \\
\hline $\mathrm{Mo}(\mathrm{NAr})(\mathrm{CMePh})\left(\mathrm{OC}_{6} \mathrm{~F}_{5}\right)_{2}(\mathrm{THF})_{\mathrm{x}}$ & 32 & 48 & $>99$ & $23 / 77$ \\
\hline
\end{tabular}




\section{X-Ray Crystallography}

General Considerations. Diffraction data were collected on a Bruker-AXS Apex II diffractometer with an Apex II CCD detector using Mo $K_{\alpha}$ radiation $(\lambda=0.71073 \AA)$ from a fine-focus sealed tube source. Data were collected at $100 \mathrm{~K}$ by performing $0.5^{\circ} \omega$-scans, integrated using SAINT ${ }^{1}$, and absorption corrected using SADABS ${ }^{2}$. The structure was solved by direct methods using SHELXT $^{3}$ and refined against $F^{2}$ on all data by full-matrix least squares with SHELXL-2018/3 following established refinement strategies. ${ }^{5}$ All non-hydrogen atoms were refined anisotropically. All hydrogen atoms were included into the model at geometrically calculated positions and refined using a riding model. The isotropic displacement parameters of all hydrogen atoms were fixed to 1.2 times the $U$ value of the atoms they are linked to (1.5 times for methyl groups). Crystal and data quality details, as well as a summary of the residual refinement values, are listed in the accompanying table.

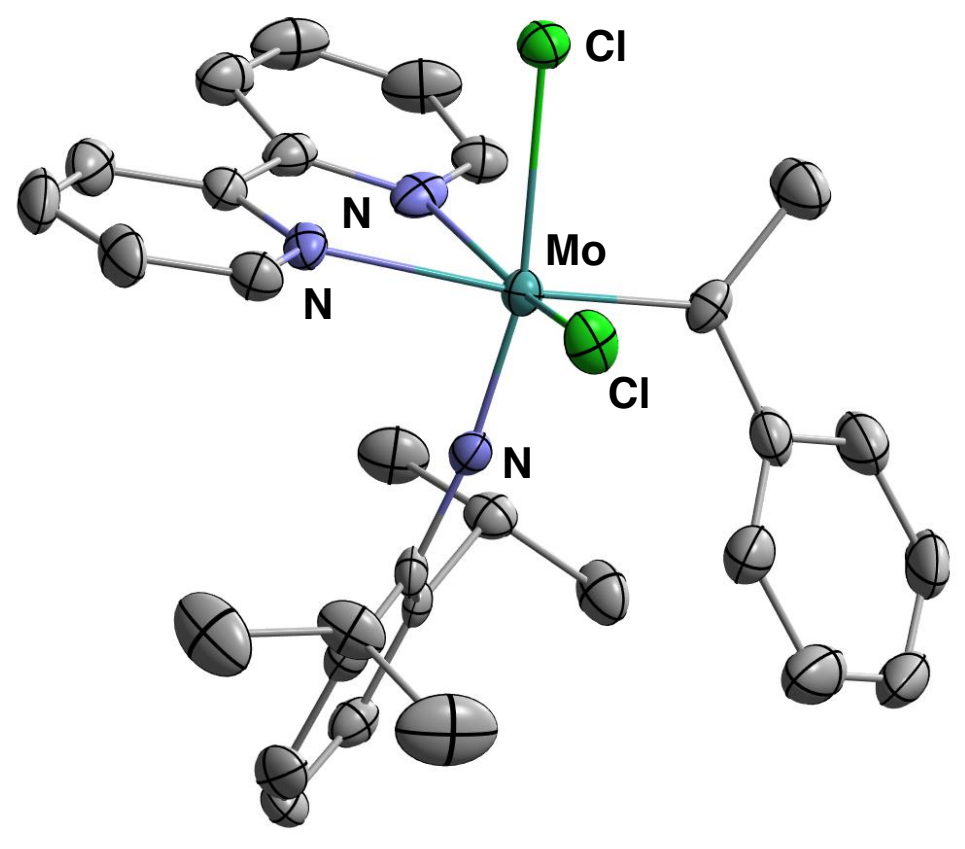

Figure S36. Thermal ellipsoid plot $(50 \%)$ of $\mathrm{Mo}(\mathrm{NAr})(\mathrm{CMePh})(\mathrm{Cl})_{2}$ (bipy). Unlabeled gray ellipsoids correspond to carbon. Solvent molecules and hydrogen atoms have been omitted for clarity. 
Table S3. Crystal data and structure refinement for $\mathrm{Mo}(\mathrm{NAr})(\mathrm{CMePh})(\mathrm{OMes})_{2}(\mathbf{1})$.

Identification code

Empirical formula

Formula weight

Temperature

Wavelength

Crystal system

Space group

Unit cell dimensions

Volume

Z

Density (calculated)

Absorption coefficient

$F(000)$

Crystal size

$\theta$ range for data collection

Index ranges

Reflections collected

Independent reflections

Completeness to $\theta=25.242^{\circ}$

Absorption correction

Refinement method

Data / restraints / parameters

Goodness-of-fit on $F^{2}$

Final $R$ indices [I $\left.>2 \sigma_{1}\right]$

$R$ indices (all data, $0.69 \AA$ )

Largest diff. peak and hole rrs $8 \mathrm{jt}$

$\mathrm{C}_{38} \mathrm{H}_{47} \mathrm{MoNO}_{2}$

$645.70 \mathrm{~g} / \mathrm{mol}$

100(2) K

$0.71073 \AA$

Monoclinic

$\mathrm{P} 2{ }_{1} / \mathrm{c}$

$$
\begin{array}{ll}
a=17.3459(4) \AA & \alpha=90^{\circ} \\
b=10.2404(3) \AA & \beta=91.4203(11)^{\circ} \\
c=18.9524(5) \AA & \gamma=90^{\circ}
\end{array}
$$

$3365.46(15) \AA^{3}$

4

$1.274 \mathrm{mg} / \mathrm{m}^{3}$

$0.422 \mathrm{~mm}^{-1}$

1360

$0.295 \times 0.290 \times 0.092 \mathrm{~mm}^{3}$

2.150 to $30.996^{\circ}$

$-25 \leq h \leq 25,-14 \leq k \leq 14,-27 \leq l \leq 27$

108793

$10720\left[R_{\text {int }}=0.0364\right]$

$100.0 \%$

Semi-empirical from equivalents

Full-matrix least-squares on $F^{2}$

10720 / 0 / 390

1.031

$R_{1}=0.0248, w R_{2}=0.0613$

$R_{1}=0.0309, w R_{2}=0.0648$

0.530 and $-0.459 \mathrm{e} / \AA^{3}$ 
Table S4. Crystal data and structure refinement for $\mathrm{Mo}(\mathrm{NAr})(\mathrm{CMePh})\left(\mathrm{OC}_{6} \mathrm{~F}_{5}\right)_{2}(\mathrm{MeCN})$ $(2(\mathrm{MeCN}))$.

\begin{tabular}{|c|c|c|}
\hline Identification code & rrs17jt & \\
\hline Empirical formula & $\mathrm{C}_{39} \mathrm{H}_{40} \mathrm{~F}_{10} \mathrm{MoN}_{2} \mathrm{O}_{2}$ & \\
\hline Formula weight & $854.67 \mathrm{~g} / \mathrm{mol}$ & \\
\hline Temperature & $100(2) \mathrm{K}$ & \\
\hline Wavelength & $0.71073 \AA$ & \\
\hline Crystal system & Monoclinic & \\
\hline Space group & $\mathrm{P} 2_{1} / \mathrm{n}$ & \\
\hline Unit cell dimensions & $a=10.2161(3) \AA$ & $\alpha=90^{\circ}$ \\
\hline & $b=18.5134(5) \AA$ & $\beta=99.2977(12)^{\circ}$ \\
\hline & $c=20.5418(5) \AA$ & $\gamma=90^{\circ}$ \\
\hline Volume & $3834.12(18) \AA^{3}$ & \\
\hline Z & 4 & \\
\hline Density (calculated) & $1.481 \mathrm{mg} / \mathrm{m}^{3}$ & \\
\hline Absorption coefficient & $0.427 \mathrm{~mm}^{-1}$ & \\
\hline$F(000)$ & 1744 & \\
\hline Crystal size & $0.339 \times 0.157 \times 0.1$ & \\
\hline$\theta$ range for data collection & 2.009 to $27.876^{\circ}$ & \\
\hline Index ranges & $-13 \leq h \leq 13,-24$ & $4,-27 \leq l \leq 27$ \\
\hline Reflections collected & 127521 & \\
\hline Independent reflections & $9151\left[R_{\text {int }}=0.0551\right.$ & \\
\hline Completeness to $\theta=25.242^{\circ}$ & $100.0 \%$ & \\
\hline Absorption correction & Semi-empirical fro & ivalents \\
\hline Refinement method & Full-matrix least-s & on $F^{2}$ \\
\hline Data / restraints / parameters & $9151 / 1660$ / 799 & \\
\hline Goodness-of-fit on $F^{2}$ & 1.307 & \\
\hline Final $R$ indices $\left[\mathrm{I}>2 \sigma_{1}\right]$ & $R_{1}=0.0575, w R_{2}=$ & \\
\hline$R$ indices (all data, $0.76 \AA$ ) & $R_{1}=0.0658, w R_{2}=$ & \\
\hline Largest diff. peak and hole & 0.919 and $-1.396 \mathrm{e}$ & \\
\hline
\end{tabular}

Note: Compound rrs 17 jt crystallizes in the monoclinic centrosymmetric space group $\mathrm{P} 2{ }_{1} / \mathrm{n}$ with one molecule of rrs17jt and one molecule of pentane per asymmetric unit.

Both perfluorophenoxide ligands as well as the alkylidene ligand and the pentane solvent molecule each exhibited disorder that was modeled over two positions; all disorder ratios were refined freely, and the correlated disorder of the alkylidene ligand and one perfluorophenoxide ligand were refined on the same free variable. All disorders were refined with the help of similarity restraints on 1,2- and 1,3- distances as well as similarity and rigid bond restraints for anisotropic displacement parameters. Flatness restraints were used on two minor-component phenyl rings, isotropic approximation restraints were applied to the anisotropic displacement parameters of two poorly-behaved carbon atoms within the disordered pentane molecule, and the anisotropic displacement parameters of two nearly-overlapping positions of a disordered phenoxide oxygen were constrained to be equivalent. 
Table S5. Crystal data and structure refinement for $\mathrm{Mo}(\mathrm{NAr})(\mathrm{CMePh})\left(\mathrm{OC}_{6} \mathrm{~F}_{5}\right)_{2}($ bipy $)$ (2(bipy)).

Identification code

Empirical formula

Formula weight

Temperature

Wavelength

Crystal system

Space group

Unit cell dimensions

Volume

Z

Density (calculated)

Absorption coefficient

$F(000)$

Crystal size

$\theta$ range for data collection

Index ranges

Reflections collected

Independent reflections

Completeness to $\theta=25.242^{\circ}$

Absorption correction

Refinement method

Data / restraints / parameters

Goodness-of-fit on $F^{2}$

Final $R$ indices $\left[\mathrm{I}>2 \sigma_{1}\right]$

$R$ indices (all data, $0.73 \AA$ )

Largest diff. peak and hole rrs12jt_sq

$\mathrm{C}_{42} \mathrm{H}_{33} \mathrm{~F}_{10} \mathrm{MoN}_{3} \mathrm{O}_{2}$

$897.65 \mathrm{~g} / \mathrm{mol}$

100(2) K

$0.71073 \AA$

Triclinic

$\mathrm{P} \overline{1}$

$a=12.6221(4) \AA \quad \alpha=72.5631(13)^{\circ}$

$b=18.1804(5) \AA \quad \beta=82.1978(13)^{\circ}$

$c=19.4797(5) \AA \quad \gamma=74.6797(13)^{\circ}$

4105.4(2) $\AA^{3}$

4

$1.452 \mathrm{mg} / \mathrm{m}^{3}$

$0.404 \mathrm{~mm}^{-1}$

1816

$0.329 \times 0.122 \times 0.062 \mathrm{~mm}^{3}$

1.098 to $29.130^{\circ}$

$-17 \leq h \leq 17,-24 \leq k \leq 24,-26 \leq l \leq 26$

151607

$22083\left[R_{\text {int }}=0.0515\right]$

$100.0 \%$

Semi-empirical from equivalents

Full-matrix least-squares on $F^{2}$

22083 / 0 / 1055

1.019

$R 1=0.0300, w R 2=0.0678$

$R 1=0.0479, w R 2=0.0757$

0.571 and $-0.523 \mathrm{e} / \AA^{3}$ 
Table S6. Crystal data and structure refinement for $\mathrm{Mo}(\mathrm{NAr})(\mathrm{CMePh})(\mathrm{Cl})_{2}$ (bipy) (3(bipy)).

Identification code

Empirical formula

Formula weight

Temperature

Wavelength

Crystal system

Space group

Unit cell dimensions

Volume

Z

Density (calculated)

Absorption coefficient

$F(000)$

Crystal size

$\theta$ range for data collection

Index ranges

Reflections collected

Independent reflections

Completeness to $\theta=25.242^{\circ}$

Absorption correction

Refinement method

Data / restraints / parameters

Goodness-of-fit on $F^{2}$

Final $R$ indices $\left[\mathrm{I}>2 \sigma_{1}\right]$

$R$ indices (all data, $0.75 \AA$ )

Absolute structure parameter

Largest diff. peak and hole rrs11jt

$\mathrm{C}_{67} \mathrm{H}_{74} \mathrm{Cl}_{4} \mathrm{Mo}_{2} \mathrm{~N}_{6}$

$1297.00 \mathrm{~g} / \mathrm{mol}$

100(2) K

$0.71073 \AA$

Orthorhombic

Fdd2

$a=31.8856(12) \AA \quad \alpha=90^{\circ}$

$b=37.8599(14) \AA \quad \beta=90^{\circ}$

$c=10.3398(4) \AA \quad \gamma=90^{\circ}$

12482.1(8) $\AA^{3}$

8

$1.380 \mathrm{mg} / \mathrm{m}^{3}$

$0.618 \mathrm{~mm}^{-1}$

5360

$0.335 \times 0.028 \times 0.020 \mathrm{~mm}^{3}$

1.670 to $28.278^{\circ}$

$-42 \leq h \leq 28,-50 \leq k \leq 49,-12 \leq l \leq 13$

30976

$7306\left[R_{\text {int }}=0.0793\right]$

$100.0 \%$

Semi-empirical from equivalents

Full-matrix least-squares on $F^{2}$

7306 / 325 / 433

1.020

$R_{1}=0.0516, w R_{2}=0.0943$

$R_{1}=0.0804, w R_{2}=0.1044$

$-0.05(3)$

0.692 and $-0.758 \mathrm{e} / \AA^{3}$ 
Table S7. Crystal data and structure refinement for $\mathrm{Mo}(\mathrm{NAr})(\mathrm{CMePh})(\mathrm{Cl})(\mathrm{OHMT})(\mathrm{MeCN})$ $(4(\mathrm{MeCN}))$.

Identification code

Empirical formula

Formula weight

Temperature

Wavelength

Crystal system

Space group

Unit cell dimensions

Volume

Z

Density (calculated)

Absorption coefficient

$F(000)$

Crystal size

$\theta$ range for data collection

Index ranges

Reflections collected

Independent reflections

Completeness to $\theta=25.242^{\circ}$

Absorption correction

Refinement method

Data / restraints / parameters

Goodness-of-fit on $F^{2}$

Final $R$ indices [I $\left.>2 \sigma_{1}\right]$

$R$ indices (all data, $0.73 \AA$ )

Largest diff. peak and hole rrs15jt

$\mathrm{C}_{51.50} \mathrm{H}_{60} \mathrm{ClMoN}_{3} \mathrm{O}$

$868.41 \mathrm{~g} / \mathrm{mol}$

100(2) K

$0.71073 \AA$

Monoclinic

$\mathrm{P} 2{ }_{1} / \mathrm{n}$

$a=8.8880(2) \AA \quad \alpha=90^{\circ}$

$b=17.1281(4) \AA \quad \beta=97.9340(12)^{\circ}$

$c=30.3238(7) \AA \quad \gamma=90^{\circ}$

4572.14(18) $\AA^{3}$

4

$1.262 \mathrm{mg} / \mathrm{m}^{3}$

$0.385 \mathrm{~mm}^{-1}$

1828

$0.205 \times 0.135 \times 0.130 \mathrm{~mm}^{3}$

1.803 to $29.130^{\circ}$

$-12 \leq h \leq 12,-23 \leq k \leq 23,-41 \leq l \leq 41$

114788

$12310\left[R_{\text {int }}=0.0851\right]$

$100.0 \%$

Semi-empirical from equivalents

Full-matrix least-squares on $F^{2}$

12310 / 415 / 603

1.025

$R_{1}=0.0405, w R_{2}=0.0923$

$R_{1}=0.0593, w R_{2}=0.1010$

0.487 and $-0.802 \mathrm{e} / \AA^{3}$ 
Table S8. Crystal data and structure refinement for $\mathrm{Mo}(\mathrm{NAr})(\mathrm{CMePh})(\mathrm{OHMT})\left(\mathrm{NC}_{4} \mathrm{H}_{4}\right)(\mathbf{5})$.

Identification code

Empirical formula

Formula weight

Temperature

Wavelength

Crystal system

Space group

Unit cell dimensions

Volume

Z

Density (calculated)

Absorption coefficient

$F(000)$

Crystal size

$\theta$ range for data collection

Index ranges

Reflections collected

Independent reflections

Completeness to $\theta=25.242^{\circ}$

Absorption correction

Refinement method

Data / restraints / parameters

Goodness-of-fit on $F^{2}$

Final $R$ indices $\left[\mathrm{I}>2 \sigma_{1}\right]$

$R$ indices (all data, $0.80 \AA$ )

Largest diff. peak and hole rrs16jt

$\mathrm{C}_{48} \mathrm{H}_{54} \mathrm{MoN}_{2} \mathrm{O}$

$770.87 \mathrm{~g} / \mathrm{mol}$

100(2) K

$0.71073 \AA$

Monoclinic

$\mathrm{P} 21 / \mathrm{n}$

$a=9.0923(2) \AA$ $\alpha=90^{\circ}$

$b=20.8485(6) \AA$

$\beta=96.8195(13)^{\circ}$

$c=21.7949(6) \AA$

$\gamma=90^{\circ}$

4102.23(19) $\AA^{3}$

4

$1.248 \mathrm{mg} / \mathrm{m}^{3}$

$0.357 \mathrm{~mm}^{-1}$

1624

$0.199 \times 0.140 \times 0.040 \mathrm{~mm}^{3}$

1.882 to $26.372^{\circ}$

$-11 \leq h \leq 11,-26 \leq k \leq 26,-27 \leq l \leq 27$

53660

$8406\left[R_{\text {int }}=0.0830\right]$

$100.0 \%$

Semi-empirical from equivalents

Full-matrix least-squares on $F^{2}$

8406 / 0 / 480

1.022

$R_{1}=0.0375, w R_{2}=0.0785$

$R_{1}=0.0594, w R_{2}=0.0880$

0.458 and $-0.423 \mathrm{e} / \AA^{3}$ 


\section{References}

1 Rieth, R. D.; Mankad, N. P.; Calimano, E.; Sadighi, J. P. Palladium-Catalyzed Cross-

Coupling of Pyrrole Anions with Aryl Chlorides, Bromides, and Iodides. Org. Lett. 2004, 6, 3981-3983.

2 Peryshkov, D. V.; Schrock, R. R.; Takase, M. K.; Muller, P.; Hoveyda, A. H. Z-Selective Olefin Metathesis Reactions Promoted by Tungsten Oxo Alkylidene Complexes. J. Am. Chem. Soc. 2011, 133, 20754-20757.

3 Schrock, R. R.; Murdzek, J. S.; Bazan, G. C.; Robbins, J.; DiMare, M.; O'Regan, M. Synthesis of Molybdenum Imido Alkylidene Complexes and Some Reactions Involving Acyclic Olefins. J. Am. Chem. Soc. 1990, 112, 3875-3886.

4 SAINT, version 8.34A, Bruker (2012), Bruker AXS Inc., Madison, Wisconsin, USA.

5 SADABS, version 2012/1, Bruker (2012), Bruker AXS Inc., Madison, Wisconsin, USA.

6 Sheldrick, G. M., Acta Cryst. 2015, A71, 3-8.

$7 \quad$ Sheldrick, G. M., Acta Cryst. 2015, C71, 3-8.

$8 \quad$ Müller, P. Crystallography Reviews 2009, 15, 57-83. 